\title{
Notes on massless scalar field partition functions, modular invariance and Eisenstein series
}

\author{
Francesco Alessio, $^{a, b, c}$ Glenn Barnich $^{b}$ and Martin Bonte ${ }^{b}$ \\ ${ }^{a}$ Nordita, KTH Royal Institute of Technology and Stockholm University, \\ Hannes Alfvéns väg 12, SE-106 91 Stockholm, Sweden \\ ${ }^{b}$ Physique Théorique et Mathématique, Université libre de Bruxelles and \\ International Solvay Institutes, \\ Campus Plaine C.P. 231, B-1050 Bruxelles, Belgium \\ "Dipartimento di Fisica "E. Pancini" and INFN Università degli studi di Napoli "Federico II", \\ I-80125 Napoli, Italy \\ E-mail: francesco.alessio@su.se, gbarnich@ulb.ac.be, mbonte@ulb.ac.be
}

ABSTRACT: The partition function of a massless scalar field on a Euclidean spacetime manifold $\mathbb{R}^{d-1} \times \mathbb{T}^{2}$ and with momentum operator in the compact spatial dimension coupled through a purely imaginary chemical potential is computed. It is modular covariant and admits a simple expression in terms of a real analytic $\mathrm{SL}(2, \mathbb{Z})$ Eisenstein series with $s=(d+1) / 2$. Different techniques for computing the partition function illustrate complementary aspects of the Eisenstein series: the functional approach gives its series representation, the operator approach yields its Fourier series, while the proper time/heat kernel/world-line approach shows that it is the Mellin transform of a Riemann theta function. High/low temperature duality is generalized to the case of a non-vanishing chemical potential. By clarifying the dependence of the partition function on the geometry of the torus, we discuss how modular covariance is a consequence of full $\mathrm{SL}(2, \mathbb{Z})$ invariance. When the spacetime manifold is $\mathbb{R}^{p} \times \mathbb{T}^{q+1}$, the partition function is given in terms of a $\mathrm{SL}(q+1, \mathbb{Z})$ Eisenstein series again with $s=(d+1) / 2$. In this case, we obtain the high/low temperature duality through a suitably adapted dual parametrization of the lattice defining the torus. On $\mathbb{T}^{d+1}$, the computation is more subtle. An additional divergence leads to an harmonic anomaly.

Keywords: Anomalies in Field and String Theories, Conformal Field Theory, Discrete Symmetries

ArXIV EPrint: 2111.03164 


\section{Contents}

1 Introduction 1

2 Generalities. One small spacetime dimension 3

2.1 Mode expansion 3

2.2 Canonical approach to partition function in large volume limit 4

2.2.1 Zero mode contribution 5

2.2.2 Oscillator contribution 5

2.3 Functional approach and zeta function 6

2.4 Functional approach to partition function in large volume limit 7

$\begin{array}{ll}2.5 & \text { Schwinger proper time, heat kernel and worldline approach }\end{array}$

2.6 Worldline approach to partition function in the large volume limit 10

2.7 Casimir energy on $\mathbb{R}^{d-1} \times \mathbb{S}^{1} \quad 11$

3 Two small spacetime dimensions and Eisenstein series 13

$\begin{array}{lll}3.1 & \text { Functional approach } & 13\end{array}$

$\begin{array}{ll}3.2 \text { Canonical approach } & 18\end{array}$

$\begin{array}{lll}3.3 & \text { Worldline approach } & 23\end{array}$

$\begin{array}{lll}3.4 & \text { One spatial dimension } & 25\end{array}$

$\begin{array}{lll}3.4 .1 & \text { Canonical approach } & 25\end{array}$

$\begin{array}{lll}3.4 .2 & \text { Dimensional continuation } & 26\end{array}$

4 Higher dimensional tori $\quad 28$

$\begin{array}{lll}4.1 & \text { Generalities } & 28\end{array}$

$\begin{array}{llr}4.2 & \text { Functional approach } & 29\end{array}$

$\begin{array}{lll}4.3 & \text { Canonical approach } & 33\end{array}$

4.4 Worldline approach 44

4.5 Partition function on $\mathbb{T}^{d+1}$ and harmonic anomaly 46

$\begin{array}{lll}5 & \text { Outlook } & 48\end{array}$

A $\operatorname{GL}(n, \mathbb{R}) / \operatorname{SO}(n, \mathbb{R})$ and $\operatorname{SL}(n, \mathbb{R}) / \operatorname{SO}(n, \mathbb{R})$ Laplacians $\quad 49$

B Trigonometric expansions $\quad 52$ 


\section{Introduction}

The $\mathrm{SL}(2, \mathbb{Z})$ real analytic Eisenstein series,

$$
f_{s}(\tau, \bar{\tau})=\sum_{(m, n) \in \mathbb{Z}^{2} /(0,0)} \frac{\tau_{2}^{s}}{|m+n \tau|^{2 s}}, \quad \mathfrak{R e}(s)>1,
$$

with $\tau=\tau_{1}+i \tau_{2} \in \mathbb{C}$, invariant under modular transformations

$$
\tau \rightarrow \frac{a \tau+b}{c \tau+d}, \quad a, b, c, d \in \mathbb{Z}, \quad a d-b c=1,
$$

feature prominently in theoretical physics, for instance in the context of string theory amplitudes, gravitational instantons and Feynman integrals, see e.g. [1-5] and references therein.

The purpose of the first part of these notes is to review how such series directly appear in the partition function of a real massless scalar field living on a Euclidean spacetime manifold with two small dimensions with periodic boundary conditions. One of these small dimensions is the Euclidean time, the other being one of the spatial dimensions, while the remaining $d-1$ spatial dimensions are taken to be large. When $d=1$, one recovers the well-known modular invariant result.

Casimir energies and partition functions for such configurations were originally computed in [6] in terms of Epstein zeta functions (see also [7, 8] for worldline methods and [9-11] for further developments and reviews). This result has also been derived in the context of conformal field theories in higher dimensions in [12]. The aim there was an attempt to generalize some of the successes of two dimensional conformal field theories in general and of modular invariance, and thus of high/low temperature duality, in particular [13-15] to higher dimensions [16]. More recent investigations along these lines include [17-20].

Our motivation for re-analyzing these scalar field results comes from a recent investigation on the implications of boundary or fall-off conditions for both the degrees of freedom and the partition functions of gauge and gravitational theories [21, 22]. More concretely, we have studied how a modular covariant partition function can be constructed for electromagnetism in the context of the Casimir set-up, that is to say for a slab geometry with two perfectly conducting parallel and infinitely extended metal plates separated by a distance a. We have shown how the well-known $E$ and $H$ modes (see e.g. [23] for an early review) may be combined into a single massless scalar field with periodic boundary conditions on an interval of length $2 a$. Modular covariance for electromagnetism then follows from the scalar field result when understanding what electromagnetic operator corresponds to scalar field momentum in the compact dimension [24]. Moreover, this analysis may be generalized directly to the case of gravitons [25].

In the second part of these notes, we compute the partition function on the flat Euclidean spacetime manifold $\mathbb{R}^{d-q} \times \mathbb{T}^{q+1}$ in terms of a $\mathrm{SL}(q+1, \mathbb{Z})$ Eisenstein series with $s=\frac{d+1}{2}$. On a skewed torus, the periodicities of the scalar field are described by general lattice vectors. A key point is to introduce adapted coordinates in which these periodicities become equal to unity along each direction. In these coordinates, the information on the torus is encoded in the coupling of the scalar field to a flat background metric that contains the information 
on the geometry of the torus. The more skewed the torus, the more observables are turned on in the partition function. High/low temperature duality follows by two complementary $\mathrm{ADM}$ parametrizations of this metric. For the partition function on $\mathbb{T}^{d+1}$, the result is divergent. As understood in [1], using dimensional regularization, the subtraction of the pole leads to an harmonic anomaly.

The literature on partition functions for massless scalar fields and its dependence on boundary conditions on the one hand, and on physical applications of Eisenstein series on the other, is substantial. Hence, the question on the added value of the current discussion, which necessarily has considerable overlaps with existing literature, is legitimate.

(i) From the viewpoint of the finite temperature Casimir effect, our discussion and techniques follow closely the approach developed in [26-29] in order to derive and understand temperature inversion symmetry, originally discovered in [30] through the method of images, in terms of functional methods and Epstein zeta functions. Our addition here consists in including a chemical potential for linear momentum in the compact dimension which brings one from Epstein zeta functions with temperature inversion symmetry to real analytic SL $(2, \mathbb{Z})$ Eisenstein series with modular covariance.

(ii) From the viewpoint of modular transformations in higher dimensions, as compared to the analysis in [12], our derivation provides the full analytic expression for the partition function in the case of the simplest model of a free massless scalar field on the flat Euclidean manifold $\mathbb{R}^{p} \times \mathbb{T}^{q+1}$ for $p+q=d$, in terms of $\operatorname{SL}(q+1, \mathbb{Z})$ Eisenstein series, with an explicit proof of $\operatorname{SL}(q+1, \mathbb{Z})$ invariance built in (see equation (4.22) and remark (iii) thereafter). As compared to [18], for the model under consideration, there is full control on finite-size corrections, and also a new formula, valid now at low rather than at high temperature, that relates the leading contribution to the entropy to the Casimir energy density of a massless scalar field in one dimension lower (see equations (4.66) and (4.74)). With respect to [1], the high temperature/large volume discussion is extended from a factorized torus $\mathbb{T}^{q} \times \mathbb{S}^{1}$ to a general torus $\mathbb{T}^{q+1}$ through a dual ADM parametrization (see equations (4.85) and (4.88)).

(iii) From the viewpoint of the relevance of Eisenstein series in physical applications, we show that, in the context of quantum statistical physics, partitions functions of massless scalar fields on Euclidean spacetime manifolds of the form $\mathbb{R}^{p} \times \mathbb{T}^{q+1}$ are among the simplest physical observables that are directly expressed through such series. Various approaches to computing these partition functions, such as functional integrals, canonical quantization or proper-time/heat kernel/world-line methods illustrate complementary aspects of Eisenstein series.

The plan of the paper is the following. In the next section, we briefly review how to derive the scalar black body result, i.e., the partition function for a massless scalar field in $d$ large spatial dimensions by canonical, path integral and proper time methods. At the end of the section, we recall how this black body partition function can be used to derive the Casimir energy at zero temperature in the case of one small spatial dimension. 
We then turn to the case of finite temperature with one small spatial dimension where the partition function can be simply expressed in terms of the Eisenstein series $f_{(d+1) / 2}$ when turning on a purely imaginary chemical potential for the momentum operator in the small dimension. Our discussion covers the Casimir effect at finite and zero temperature.

In the last section, we generalize the discussion to $p$ large and $q=d-p$ small spatial dimensions. We clarify how the partition function depends on the full geometry of the $q+1$ dimensions torus, including the case $q=1$, and express the result in terms of an $\mathrm{SL}(q+1, \mathbb{Z})$ invariant partition function. All results of the previous sections may be obtained as particular cases, so that one may start directly with this section if one is interested in the final results and the general structure, but not necessarily the way our understanding of these matters has evolved from studying the simpler particular cases first.

\section{Generalities. One small spacetime dimension}

\subsection{Mode expansion}

Consider a massless scalar field $\phi$ in $d$ spatial dimensions, whose Lagrangian action reads

$$
S[\phi]=-\frac{1}{2} \int \mathrm{d} x^{0} \int_{V_{d}} \mathrm{~d}^{d} x \partial_{\mu} \phi \partial^{\mu} \phi,
$$

while the first order Hamiltonian action is

$$
S_{H}[\phi, \pi]=\int \mathrm{d} x^{0}\left[\int_{V_{d}} \mathrm{~d}^{d} x \dot{\phi} \pi-H\right], \quad H=\frac{1}{2} \int_{V_{d}} \mathrm{~d}^{d} x\left(\pi^{2}+\partial_{i} \phi \partial^{i} \phi\right),
$$

with associated canonical Poisson brackets

$$
\left\{\phi\left(x^{0}, x\right), \pi\left(x^{0}, x^{\prime}\right)\right\}=\delta^{d}\left(x-x^{\prime}\right) .
$$

Our conventions for indices and their ranges are as follows: the spacetime and spatial indices are $\mu=0, \ldots, d$, and $i=1, \ldots, d$, respectively. Besides the Hamiltonian, the other observables that are relevant for us here are linear momenta in the $x^{i}$ direction,

$$
P_{i}=-\int_{V_{d}} \mathrm{~d}^{d} x \pi \partial_{i} \phi
$$

Our aim is to discuss analytic expressions in terms of Eisenstein series and associated modular properties of partition functions of the type

$$
Z_{d}\left(\beta, \mu^{j}\right)=\operatorname{Tr} e^{-\beta\left(\hat{H}-i \mu^{j} \hat{P}_{j}\right)}
$$

and generalizations thereof. In order to take advantage of techniques in complex analysis, we use purely imaginary chemical potentials $i \mu^{j}, \mu^{j} \in \mathbb{R}$, as in 2-dimensional conformal field theory and also for instance in [31-33].

In order to evaluate such partition functions, a standard procedure is to put the system in a hyperrectangular box of volume $V_{d}=\prod_{i=1}^{d} L_{i}$ and to choose periodic boundary conditions in all spatial dimensions,

$$
\phi\left(x^{0}, x^{1}, \ldots, x^{i}, \ldots, x^{d}\right)=\phi\left(x^{0}, x^{1}, \ldots, x^{i}+L_{i}, \ldots, x^{d}\right) .
$$


The appropriate orthonormal basis vectors $\left\{e_{k_{i}}\right\}$ are plane waves,

$$
e_{k_{i}}(x)=\frac{1}{\sqrt{V_{d}}} e^{i k_{i} x^{i}}, \quad k_{i}=\frac{2 \pi n_{i}}{L_{i}}, \quad\left(e_{k_{i}}, e_{k_{i}^{\prime}}\right)=\int_{V_{d}} \mathrm{~d}^{d} x e_{k_{i}}^{*}(x) e_{k_{i}^{\prime}}(x)=\prod_{i} \delta_{n_{i}, n_{i}^{\prime}} .
$$

The fields $\phi, \pi$ admit the mode decomposition

$$
\phi(x)=\sum_{n_{i} \in \mathbb{Z}^{d}} \phi_{k_{i}} e_{k_{i}}(x), \quad \pi(x)=\sum_{n_{i} \in \mathbb{Z}^{d}} \pi_{k_{i}}(x) e_{k_{i}},
$$

with reality conditions $\phi_{k_{i}}=\phi_{-k_{i}}^{*}, \pi_{k_{i}}=\pi_{-k_{i}}^{*}$. In terms of the Fourier components, the observables are

$$
H=\frac{1}{2} \sum_{n_{i} \in \mathbb{Z}^{d}}\left(\pi_{k_{i}} \pi_{k_{i}}^{*}+\omega_{k_{i}}^{2} \phi_{k_{i}} \phi_{k_{i}}^{*}\right), \quad P_{i}=-i \sum_{n_{i} \in \mathbb{Z}^{d}} k_{i} \phi_{k_{i}} \pi_{k_{i}}^{*},
$$

where $\omega_{k_{i}}=\sqrt{k_{j} k^{j}}$, while the non-vanishing canonical Poisson brackets become

$$
\left\{\phi_{k_{i}}, \pi_{k_{i}^{\prime}}^{*}\right\}=\prod_{i} \delta_{n_{i}, n_{i}^{\prime}}
$$

The zero mode is denoted by $\phi_{0}=q, \pi_{0}=p$ and, for the Fourier components with $n_{i} \neq(0, \ldots, 0)$, one defines standard oscillator variables,

$$
a_{k_{i}}=\sqrt{\frac{\omega_{k_{i}}}{2}}\left[\phi_{k_{i}}+\frac{i}{\omega_{k_{i}}} \pi_{k_{i}}\right] \text {. }
$$

In these terms,

$$
\begin{aligned}
& \phi=V_{d}^{-\frac{d-1}{2 d}} q+\frac{1}{\sqrt{V_{d}}} \sum_{n_{i} \in \mathbb{Z}^{d}}{ }^{\prime} \frac{1}{\sqrt{2 \omega_{k_{i}}}}\left(a_{k_{i}} e^{i k_{j} x^{j}}+\text { c.c. }\right) \\
& \pi=V_{d}^{-\frac{d+1}{2 d}} p-\frac{i}{\sqrt{V_{d}}} \sum_{n_{i} \in \mathbb{Z}^{d}}^{\prime} \sqrt{\frac{\omega_{k_{i}}}{2}}\left(a_{k_{i}} e^{i k_{j} x^{j}}-\text { c.c. }\right)
\end{aligned}
$$

where the prime indicates that the term with $n_{i}=(0, \ldots, 0)$ is excluded from the sum and $q, p$ are dimensionless. The observables are given by

$$
H=V_{d}^{-\frac{1}{d}} \frac{p^{2}}{2}+\frac{1}{2} \sum_{n_{i} \in \mathbb{Z}^{d}}^{\prime} \omega_{k_{i}}\left(a_{k_{i}}^{*} a_{k_{i}}+a_{k_{i}} a_{k_{i}}^{*}\right), \quad P_{i}=\frac{1}{2} \sum_{n_{i} \in \mathbb{Z}^{d}}^{\prime} k_{i}\left(a_{k_{i}}^{*} a_{k_{i}}+a_{k_{i}} a_{k_{i}}^{*}\right),
$$

while the non-vanishing Poisson brackets become

$$
\{q, p\}=1, \quad\left\{a_{k_{i}}, a_{k_{i}}^{*}\right\}=-i \prod_{i} \delta_{n_{i}, n_{i}^{\prime}} .
$$

\subsection{Canonical approach to partition function in large volume limit}

Throughout this section, where we will consider all spatial dimensions to be large, we will also take the chemical potentials $\mu^{j}$ to vanish. How the final result changes when they are turned on will be discussed from a more general perspective later. 


\subsubsection{Zero mode contribution}

It follows from the expression of the Hamiltonian (2.13) that the zero mode sector of the theory corresponds to a free particle with Hamiltonian $H_{0}=V_{d}^{-\frac{1}{d}} \frac{1}{2} p^{2}$. Therefore one may quantize the full theory in a Fock space for which the vacua $|p\rangle$ are labeled by the continuous eigenvalues $p$ of $\hat{p}$,

$$
\hat{p}|p\rangle=p|p\rangle, \quad \hat{a}_{k_{i}}|p\rangle=0 .
$$

It follows that

$$
Z_{0}(\beta)=\operatorname{Tr} e^{-\beta \hat{H}_{0}}=\int_{-\infty}^{+\infty} \mathrm{d} p e^{-\frac{b}{2} p^{2}}\langle p \mid p\rangle=\sqrt{\frac{2 \pi}{b}} \delta(0), \quad b=V_{d}^{-\frac{1}{d}} \beta .
$$

\subsubsection{Oscillator contribution}

For the oscillator modes quantized in Fock space, one may directly evaluate the trace using the normal ordered Hamiltonian : $\hat{H}^{\prime}$ : so that no divergences occur. If $N_{k_{i}}$ denotes the occupation number of the oscillator associated to $k_{i}$, we have,

$$
Z_{d}^{\prime}(\beta)=\operatorname{Tr} e^{-\beta: \hat{H}^{\prime}:}=\prod_{n_{i} \in \mathbb{Z}^{d}}^{\prime} \sum_{N_{k_{i}} \in \mathbb{N}} e^{-\beta \omega_{k_{i}} N_{k_{i}}}=\prod_{n_{i} \in \mathbb{Z}^{d}}^{\prime} \frac{1}{1-e^{-\beta \omega_{k_{i}}}},
$$

and thus

$$
\ln Z_{d}^{\prime}(\beta)=-\sum_{n_{i} \in \mathbb{Z}^{d}}^{\prime} \ln \left(1-e^{-\beta \omega_{k_{i}}}\right)
$$

For large $L_{i}$, the sums can be approximated by integrals using $\mathrm{d} k_{i}=\frac{2 \pi}{L_{i}} \mathrm{~d} n_{i}$. After performing the integrals over the angles in hyperspherical coordinates, one finds

$$
\begin{aligned}
\ln Z_{d}^{\prime}(\beta) & =-\frac{V_{d}}{(2 \pi)^{d}} \int \mathrm{d}^{d} k \ln \left(1-e^{-\beta \omega_{k_{i}}}\right) \\
& =-\frac{V_{d}}{(2 \pi)^{d}} \operatorname{Vol}\left(\mathbb{S}^{d-1}\right) \int_{0}^{\infty} \mathrm{d} k k^{d-1} \ln \left(1-e^{-\beta k}\right) .
\end{aligned}
$$

Since the finite contribution of the zero mode $\ln Z_{0}(\beta) \sim \ln V_{d}$ is subdominant in the large volume limit, it may be neglected in most of the paper, unless otherwise specified.

On the one hand, $\operatorname{Vol}\left(\mathbb{S}^{d-1}\right)=2 \pi^{\frac{d}{2}} / \Gamma\left(\frac{d}{2}\right)$ while, on the other, the integral becomes after the change of variables $x=\beta k$ and a standard integrations by parts,

$$
\int_{0}^{\infty} \mathrm{d} k k^{d-1} \ln \left(1-e^{-\beta k}\right)=-\frac{1}{d \beta^{d}} \int_{0}^{\infty} \mathrm{d} x \frac{x^{d}}{e^{x}-1}=-\frac{1}{d \beta^{d}} \Gamma(d+1) \zeta(d+1) .
$$

One thus ends up with the "scalar black body" partition function

$$
\ln Z_{d}(\beta)=\frac{\Gamma(d) \zeta(d+1)}{2^{d-1} \pi^{\frac{d}{2}} \Gamma\left(\frac{d}{2}\right)} \frac{V_{d}}{\beta^{d}}
$$

Furthermore, using the reduplication formula

$$
\Gamma(z) \sqrt{\pi}=2^{z-1} \Gamma\left(\frac{z}{2}\right) \Gamma\left(\frac{z+1}{2}\right)
$$


at $z=d$, and the definition of the completion of the zeta function,

$$
\xi(z)=\frac{\Gamma\left(\frac{z}{2}\right) \zeta(z)}{\pi^{\frac{z}{2}}},
$$

at $z=d+1$, the result can be written as

$$
\ln Z_{d}(\beta)=\xi(d+1) \frac{V_{d}}{\beta^{d}}=\left\{\begin{array}{ll}
\frac{\pi}{6} \frac{L_{1}}{\beta} & \text { for } d=1 \\
\frac{\zeta(3)}{2 \pi} \frac{L_{1} L_{2}}{\beta^{2}} & \text { for } d=2 \\
\frac{\pi^{2}}{90} \frac{L_{1} L_{2} L_{3}}{\beta^{3}} & \text { for } d=3 \\
\vdots
\end{array} .\right.
$$

\section{Remarks}

(i) This result for the partition function is independent of the choice of boundary conditions in the large volume limit. For instance, for Dirichlet conditions $\phi\left(t, x^{1}, \ldots, 0, \ldots, x^{d}\right)=0=\phi\left(t, x^{1}, \ldots, L_{i}, \ldots, x^{d}\right)$, the appropriate basis is $e_{k_{i}}=$

$\sqrt{\frac{2^{d}}{V_{d}}} \prod_{i} \sin k_{i} x=e_{k_{i}}^{*}, k_{i}=\frac{\pi n_{i}}{L_{i}}$. In particular, there is no mode at $n_{i}=(0, \ldots, 0)$ that has to be dealt with. All mode expansions are the same except that sums and products are restricted to $n_{i}>0$. In the evaluation of the partition function after (2.18), this is compensated by the fact that $\mathrm{d} k_{i}=\frac{\pi}{L_{i}} \mathrm{~d} n_{i}$. Equivalently, the sums can be extended to $\mathbb{Z}^{d} /(0, \ldots, 0)$ while dividing by $2^{d}$, because the summands are even functions of the $n_{i}$, and the computation proceeds as before.

(ii) For $d=3$, the standard argument to get the partition functions for the electromagnetic field is to multiply the scalar field result by 2 in order to take into account the 2 independent polarizations of the photon. On account of the equivalence established in [24] of the electromagnetic field with Casimir boundary conditions and a scalar field on the double interval with periodic boundary conditions in the direction $x^{3}$, together with the independence of the results on boundary conditions in the large volume limit, this can also be obtained by the replacement $L_{3}=2 a$, where $a$ is the distance between the two parallel plates. In the case where the $x^{3}$ dimension is "small", the former argument no longer applies but the latter still does.

\subsection{Functional approach and zeta function}

The functional evaluation of partition functions [34] (see also e.g. [35, 36] for earlier connected work, [37] for the inclusion of chemical potentials and [10] for a review) starts from the Hamiltonian path integral representation

$$
Z_{d}\left(\beta, \mu^{j}\right)=\int \prod_{x^{a}} \mathrm{~d} \phi\left(x^{a}\right) \prod_{x^{a}} \frac{\mathrm{d} \pi\left(x^{a}\right)}{2 \pi} e^{-S_{H}^{E}},
$$

where $x^{a}=\left(x^{1}, \ldots, x^{d+1}\right)$ and the first order Euclidean action is

$$
S_{H}^{E}[\phi, \pi]=\int_{0}^{\beta} \mathrm{d} x^{d+1}\left[\int_{V_{d}} \mathrm{~d}^{d} x\left(-i \pi \partial_{d+1} \phi\right)+\left(H-i \mu^{j} P_{j}\right)\right] .
$$


The sum is over periodic phase space paths of period $\beta=L_{d+1}$ in Euclidean time $x^{d+1}$,

$$
\phi\left(x^{i}, x^{d+1}+\beta\right)=\phi\left(x^{i}, x^{d+1}\right), \quad \pi\left(x^{i}, x^{d+1}+\beta\right)=\pi\left(x^{i}, x^{d+1}\right) .
$$

After integration over the momenta, this leads to

$$
Z_{d}\left(\beta, \mu^{j}\right)=\left(\operatorname{det}\left[2 \pi \delta^{d+1}\left(x^{\prime}, x\right)\right]\right)^{-\frac{1}{2}} \int \prod_{x^{a}} d \phi\left(x^{a}\right) e^{-S_{L}^{E}}
$$

with

$$
S_{L}^{E}[\phi]=\frac{1}{2} \int_{V_{d+1}} \mathrm{~d}^{d+1} x\left[\left(\partial_{d+1} \phi-\mu^{j} \partial_{j} \phi\right)^{2}+\partial_{j} \phi \partial^{j} \phi\right],
$$

where the integration is now over a $(d+1)$-dimensional hyperrectangle in Euclidean space of volume $V_{d+1}=V_{d} \beta=\prod_{a=1}^{d+1} L_{a}$. Except for the replacement $\partial_{d+1} \rightarrow \partial_{d+1}-\mu^{j} \partial_{j}$, the operator in the action is minus the Laplacian in $d+1$ dimensions with periodic boundary conditions in all dimensions. Since the eigenfunctions are $\frac{1}{\sqrt{V_{d+1}}} e^{i k_{a} x^{a}}$ with $k_{d+1}=\frac{2 \pi n_{d+1}}{\beta}$, the eigenvalues are

$$
\lambda_{n_{a}}=\left[\left(\frac{2 \pi n_{d+1}}{\beta}-\sum_{j} \mu^{j} \frac{2 \pi n_{j}}{L_{j}}\right)^{2}+\sum_{i}\left(\frac{2 \pi n_{i}}{L_{i}}\right)^{2}\right] .
$$

In order to define the partition function, formally given by

$$
Z_{d}\left(\beta, \mu^{j}\right)=\prod_{n_{a} \in \mathbb{Z}^{d+1}}^{\prime} \sqrt{\frac{2 \pi}{\lambda_{n_{a}}}}=\left[\operatorname{det}\left(\frac{-\Delta_{\mu}}{2 \pi}\right)\right]^{-\frac{1}{2}},
$$

one introduces a parameter $\nu$ of dimension inverse length and considers the dimensionless operators $A_{\mu}=-\Delta_{\mu} / 2 \pi \nu^{2}$. The associated zeta function is defined by

$$
\zeta_{A_{\mu}}(s)=\sum_{n_{a} \in \mathbb{Z}^{d+1}}^{\prime} \lambda_{n_{a}}^{-s}\left(2 \pi \nu^{2}\right)^{s} .
$$

In these terms, the partition function is given by

$$
\ln Z_{d}\left(\beta, \mu^{j}\right)=\frac{1}{2} \zeta_{A_{\mu}}^{\prime}(0)=\frac{1}{2} \zeta_{-\Delta_{\mu}}^{\prime}(0)+\frac{1}{2} \ln \left(2 \pi \nu^{2}\right) \zeta_{-\Delta_{\mu}}(0) .
$$

\subsection{Functional approach to partition function in large volume limit}

Let us recover the canonical result of section 2.2 through the functional approach. In this case, in the absence of chemical potentials, $\mu^{j}=0, A_{0}=-\frac{\Delta_{0}}{2 \pi \nu^{2}}$ we again turn the sums over the $n_{i}$ into integrals,

$$
\zeta_{A_{0}}(s)=\left(2 \pi \nu^{2}\right)^{s} \frac{V_{d}}{(2 \pi)^{d}} \int \mathrm{d}^{d} k \sum_{n_{d+1} \in \mathbb{Z}}\left[\left(\frac{2 \pi n_{d+1}}{\beta}\right)^{2}+k_{i} k^{i}\right]^{-s} .
$$

From the Euclidean spacetime point of view, there is thus only one small dimension $x^{d+1}$ of length $\beta$. After performing the integrals over the angles in hyperspherical coordinates, we have

$$
\zeta_{A_{0}}(s)=\left(2 \pi \nu^{2}\right)^{s} \frac{V_{d} 2 \pi^{\frac{d}{2}}}{(2 \pi)^{d} \Gamma\left(\frac{d}{2}\right)} \int_{0}^{\infty} \mathrm{d} k k^{d-1} \sum_{n_{d+1} \in \mathbb{Z}}\left[\left(\frac{2 \pi n_{d+1}}{\beta}\right)^{2}+k^{2}\right]^{-s}
$$


The divergent integral contained in the term at $n_{d+1}=0$ is regulated through an infrared cut-off $\epsilon$,

$$
\int_{\epsilon}^{\infty} \mathrm{d} k k^{d-1-2 s}=-\frac{\epsilon^{d-2 s}}{d-2 s}, \quad \mathfrak{R e}(s)>\frac{d}{2} .
$$

This expression and its derivative with respect to $s$ both vanish at $s=0$ in the limit when $\epsilon \rightarrow 0$ and can thus be discarded. In the remaining terms, the integrals converge for $\mathfrak{R e}(s)>\frac{d}{2}$. After the change of variables, $k=\frac{2 \pi}{\beta} y$, we are left with

$$
\zeta_{A_{0}}(s)=\left(2 \pi \nu^{2}\right)^{s} \frac{V_{d} 2 \pi^{\frac{d}{2}}}{(2 \pi)^{d} \Gamma\left(\frac{d}{2}\right)}\left(\frac{2 \pi}{\beta}\right)^{d-2 s} \int_{0}^{\infty} \mathrm{d} y y^{d-1} \sum_{n_{d+1} \in \mathbb{Z}}^{\prime}\left[\left(n_{d+1}\right)^{2}+y^{2}\right]^{-s} .
$$

The additional change of variables $y=n_{d+1} \sinh x$ together with

$$
\int_{0}^{\infty} \mathrm{d} x \frac{\sinh ^{d-1}(x)}{\cosh ^{2 s-1}(x)}=\frac{1}{2} \frac{\Gamma\left(\frac{d}{2}\right) \Gamma\left(s-\frac{d}{2}\right)}{\Gamma(s)},
$$

then yields

$$
\zeta_{A_{0}}(s)=\left(2 \pi \nu^{2}\right)^{s} \frac{V_{d} 2 \pi^{\frac{d}{2}}}{(2 \pi)^{d}}\left(\frac{2 \pi}{\beta}\right)^{d-2 s} \frac{\Gamma\left(s-\frac{d}{2}\right) \zeta(2 s-d)}{\Gamma(s)},
$$

which can be written more compactly as

$$
\zeta_{A_{0}}(s)=\frac{\nu^{2 s} V_{d}}{\beta^{d-2 s}} 2^{1-s} \frac{\xi(2 s-d)}{\Gamma(s)} .
$$

Since $\frac{1}{\Gamma(s)}=s+O\left(s^{2}\right)$, it follows that $\zeta_{A_{0}}(0)=0$ and

$$
\ln Z_{d}(\beta)=\xi(-d) \frac{V_{d}}{\beta^{d}}
$$

When using the reflection formula

$$
\pi^{-\frac{z}{2}} \Gamma\left(\frac{z}{2}\right) \zeta(z)=\pi^{\frac{z-1}{2}} \Gamma\left(\frac{1-z}{2}\right) \zeta(1-z) \Longleftrightarrow \xi(z)=\xi(1-z),
$$

at $z=-d$, this agrees with $(2.24)$.

\subsection{Schwinger proper time, heat kernel and worldline approach}

The quadratic Lagrangian path integral (2.28) is formally solved by the one-loop contribution

$$
\ln Z_{d}\left(\beta, \mu^{j}\right)=-\frac{1}{2} \operatorname{Tr} \ln \frac{\delta^{2} S_{L}^{E}}{\delta \phi \delta \phi}
$$

which can be written as an integral over Schwinger proper time $t$ as

$$
\ln Z_{d}\left(\beta, \mu^{j}\right)=\frac{1}{2} \int_{0}^{\infty} \frac{\mathrm{d} t}{t} \operatorname{Tr} e^{-t \hat{\mathrm{H}}_{\mu}}
$$

after having removed the zero modes. Here

$$
\hat{\mathrm{H}}_{\mu}=\left(\hat{p}_{d+1}-\mu^{j} \hat{p}_{j}\right)^{2}+\hat{p}_{j} \hat{p}^{j}
$$


is not the field theory Hamiltonian but the one of a free particle of mass $\frac{1}{2}$ in $d+1$ (Euclidean) dimensions described by the canonical variables $\left(x^{a}, p_{a}\right)$. In terms of the operator

$$
\hat{T}_{\mu}(t)=e^{-t \hat{\mathrm{H}}_{\mu}}, \quad-\frac{\mathrm{d}}{\mathrm{d} t} \hat{T}_{\mu}(t)=\hat{\mathrm{H}}_{\mu} \hat{T}_{\mu}(t),
$$

the relation to the zeta function is

$$
\zeta_{\hat{\mathrm{H}}_{\mu}}(s)=\frac{1}{\Gamma(s)} \int_{0}^{\infty} \mathrm{d} t t^{s-1} \operatorname{Tr}\left(\hat{T}_{\mu}(t)-\hat{\Pi}\right),
$$

where $\hat{\Pi}$ denotes the orthogonal projector onto $\operatorname{Ker} \hat{\mathrm{H}}_{\mu}$, Tr $\hat{\Pi}=\operatorname{dim} \operatorname{Ker} \hat{\mathrm{H}}_{\mu}$.

\section{Remarks}

(i) If one takes vanishing chemical potentials $\mu^{j}=0$ and considers infinite Euclidean spacetime, which means in particular zero temperature, $\beta=L_{d+1} \rightarrow \infty$, the matrix element

$$
K_{-\Delta_{0}}\left(x^{\prime}, x ; t\right)=\left\langle x^{\prime}|\hat{T}(t)| x\right\rangle
$$

is called the heat kernel since it satisfies the heat equation

$$
\frac{\mathrm{d}}{\mathrm{d} t} K_{-\Delta_{0}}\left(x^{\prime}, x ; t\right)=\Delta_{0} K_{-\Delta_{0}}\left(x^{\prime}, x ; t\right), \quad K_{-\Delta_{0}}\left(x^{\prime}, x ; 0\right)=\delta^{d+1}\left(x^{\prime}-x\right) .
$$

The solution is well-known and can be obtained for instance by working out the (Euclidean) path integral for the transition amplitude of a particle of mass $m=\frac{1}{2}$ in $d+1$ dimensions:

$$
K_{-\Delta_{0}}\left(x^{\prime}, x ; t\right)=\frac{1}{(4 \pi t)^{\frac{d+1}{2}}} e^{-\frac{1}{4 t}\left(x_{a}^{\prime}-x_{a}\right)\left(x^{\prime a}-x^{a}\right)} .
$$

(ii) If one takes vanishing chemical potentials $\mu^{j}$ and only one spacetime dimension with finite $\beta$ instead, the periodic boundary condition, $\phi(0)=\phi(\beta)$, requires one to solve the heat kernel for a particle on a circle. The first form of the solution may be obtained by inserting a resolution of the identity in terms of eigenstates of the Laplacian.

$$
\varphi_{n}(x)=\langle x \mid n\rangle=\frac{1}{\sqrt{\beta}} e^{i \omega_{n} x}, \quad \omega_{n}=\frac{2 \pi}{\beta} n,
$$

so that

$$
K_{-\Delta_{P}}\left(x^{\prime}, x ; t\right)=\sum_{n \in \mathbb{Z}} e^{-t \omega_{n}^{2}} \varphi_{n}\left(x^{\prime}\right) \varphi_{n}^{*}(x)
$$

and may be expressed in terms of the Jacobi theta function defined by

$$
\vartheta_{3}(z \mid \tau)=\sum_{n \in \mathbb{Z}} e^{\pi i n^{2} \tau+2 \pi i n z}
$$

as follows

$$
K_{-\Delta_{P}}\left(x^{\prime}, x ; t\right)=\frac{1}{\beta} \sum_{n \in \mathbb{Z}} e^{-t\left(\frac{2 \pi n}{\beta}\right)^{2}+2 \pi i n \frac{x^{\prime}-x}{\beta}}=\frac{1}{\beta} \vartheta_{3}\left(\frac{x^{\prime}-x}{\beta} \mid i \frac{4 \pi}{\beta^{2}} t\right) .
$$


The second form of the solution follows by considering a path integral including paths that wind $m$ times around the circle of length $\beta$,

$$
K_{-\Delta_{P}}\left(x^{\prime}, x ; t\right)=\frac{1}{\sqrt{4 \pi t}} \sum_{m \in \mathbb{Z}} e^{-\frac{1}{4 t}\left(x^{\prime}-x+\beta m\right)^{2}} .
$$

Both expressions are related through the Poisson summation formula,

$$
\begin{aligned}
& \sum_{m^{\alpha} \in \mathbb{Z}^{n}} e^{-\pi\left(m^{\alpha}+y^{\alpha}\right) A_{\alpha \beta}\left(m^{\beta}+y^{\beta}\right)+2 \pi i m^{\alpha} z_{\alpha}} \\
& \quad=\frac{1}{\sqrt{\operatorname{det} A}} \sum_{n_{\alpha} \in \mathbb{Z}^{n}} e^{-\pi\left(n_{\alpha}+z_{\alpha}\right)\left(A^{-1}\right)^{\alpha \beta}\left(n_{\beta}+z_{\beta}\right)-2 \pi i\left(n_{\alpha}+z_{\alpha}\right) y^{\alpha}},
\end{aligned}
$$

in the simple case where $n=1$. For later use, one also defines

$$
\theta(t)=\vartheta_{3}(0 \mid i t)=\sum_{n \in \mathbb{Z}} e^{-t \pi n^{2}}
$$

with modular transformation

$$
\vartheta_{3}\left(0 \mid-\frac{1}{\tau}\right)=\sqrt{-i \tau} \vartheta_{3}(0, \tau) \Longrightarrow \theta\left(\frac{1}{t}\right)=\sqrt{t} \theta(t),
$$

in terms of which both expressions for the trace of the heat kernel,

$$
K_{-\Delta_{P}}(t)=\int_{0}^{\beta} \mathrm{d} x K_{-\Delta_{P}}(x, x ; t)
$$

of a particle on the circle may be written compactly as

$$
K_{-\Delta_{P}}(t)=\theta\left(\frac{4 \pi}{\beta^{2}} t\right)=\frac{\beta}{\sqrt{4 \pi t}} \theta\left(\frac{\beta^{2}}{4 \pi t}\right) .
$$

\subsection{Worldline approach to partition function in the large volume limit}

The full heat kernel in the large volume limit is obtained by combining the heat kernel in (2.50) with $d \rightarrow d-1$ for the large spatial dimensions with that in (2.54),

$$
\left\langle x^{\prime}\left|\hat{T}_{0}(t)\right| x\right\rangle=\frac{1}{\beta(4 \pi t)^{\frac{d}{2}}} e^{-\frac{1}{4 t}\left(x_{i}^{\prime}-x_{i}\right)\left(x^{\prime i}-x^{i}\right)} \vartheta_{3}\left(\frac{x_{d+1}^{\prime}-x_{d+1}}{\beta} ; i \frac{4 \pi}{\beta^{2}} t\right) .
$$

According to (2.44), the partition function in the large volume limit is

$$
\ln Z_{d}(\beta)=\frac{V_{d}}{2^{d+1} \pi^{\frac{d}{2}}} \sum_{n_{d+1} \in \mathbb{Z}}^{\prime} \int_{0}^{\infty} \mathrm{d} t t^{-\frac{d}{2}-1} e^{-t\left(\frac{2 \pi n d+1}{\beta}\right)^{2}} .
$$

After the change of variables $t \rightarrow\left(\frac{2 \pi}{\beta}\right)^{2} t$, this becomes

$$
\ln Z_{d}(\beta)=\frac{V_{d}}{\beta^{d}} \frac{\pi^{\frac{d}{2}}}{2} \sum_{n_{d+1} \in \mathbb{Z}}^{\prime} \int_{0}^{\infty} \mathrm{d} t t^{-\frac{d}{2}-1} e^{-t n_{d+1}^{2}}
$$


which may be written in terms of the theta function as

$$
\ln Z_{d}(\beta)=\frac{V_{d}}{\beta^{d}} \int_{0}^{\infty} \mathrm{d} t t^{-\frac{d}{2}-1} \frac{1}{2}(\theta(t)-1) .
$$

Going back to (2.63) and assuming that $d<-2$, one may use

$$
\frac{1}{\Gamma(s)} \int_{0}^{\infty} \mathrm{d} t t^{s-1} e^{-t \lambda}=\frac{1}{\lambda^{s}}, \quad \mathfrak{R e}(s)>0,
$$

with $\lambda>0$, so that

$$
\frac{\pi^{\frac{d}{2}}}{2} \sum_{n_{d+1} \in \mathbb{Z}}^{\prime} \int_{0}^{\infty} \mathrm{d} t t^{-\frac{d}{2}-1} e^{-t n_{d+1}^{2}}=\pi^{\frac{d}{2}} \Gamma\left(-\frac{d}{2}\right) \zeta(-d)=\xi(-d) .
$$

It follows that (2.63) agrees with (2.41). Again, the result for positive integer $d$ is obtained after using the reflection formula.

When using (2.55) instead of (2.54), the full heat kernel in the large volume limit is instead

$$
\left\langle x^{\prime}\left|\hat{T}_{0}(t)\right| x\right\rangle=\frac{1}{(4 \pi t)^{\frac{d+1}{2}}} e^{-\frac{1}{4 t}\left(x_{i}^{\prime}-x_{i}\right)\left(x^{i}-x^{i}\right)} \sum_{m^{d+1} \in \mathbb{Z}} e^{-\frac{1}{4 t}\left(x^{\prime}-x+\beta m^{d+1}\right)^{2}} .
$$

For the partition function, after dropping the $m^{d+1}=0$ term, this gives

$$
\ln Z_{d}(\beta)=\frac{V_{d} \beta}{2^{d+2} \pi^{\frac{d+1}{2}}} \sum_{m^{d+1} \in \mathbb{Z}}^{\prime} \int_{0}^{\infty} d t t^{-\frac{d+3}{2}} e^{-\frac{1}{4 t}\left(m^{d+1} \beta\right)^{2}} .
$$

After the change of variables $t \rightarrow \frac{\beta^{2}}{4 t}$, one now gets directly

$$
\ln Z_{d}(\beta)=\frac{V_{d}}{\beta^{d}} \frac{1}{2 \pi^{\frac{d+1}{2}}} \sum_{m^{d+1} \in \mathbb{Z}}^{\prime} \int_{0}^{\infty} \mathrm{d} t t^{\frac{d+1}{2}-1} e^{-t\left(m^{d+1}\right)^{2}}=\frac{V_{d}}{\beta^{d}} \xi(d+1),
$$

in agreement with the result obtained from canonical quantization in (2.24).

In terms of the theta function, the first expression for the partition function in (2.69) is

$$
\ln Z_{d}(\beta)=\frac{V_{d}}{\beta^{d}} \int_{0}^{\infty} \mathrm{d} t t^{\frac{d+1}{2}-1} \frac{1}{2}(\theta(t)-1) \text {. }
$$

In other words, equivalence of the canonical and worldline approach for the partition function shows for $z=d+1$ that the completion of the zeta function is the Mellin transform of $\frac{1}{2}(\theta(t)-1)$,

$$
\xi(z)=\frac{1}{2} \int_{0}^{\infty} \mathrm{d} t t^{\frac{z}{2}-1}(\theta(t)-1) .
$$

\subsection{Casimir energy on $\mathbb{R}^{d-1} \times \mathbb{S}^{1}$}

Suppose now that the spatial dimension $x^{d}$ is small, while all other spatial dimensions $x^{I}$, for $I=1, \ldots, d-1$ are large, $L_{I} \gg L_{d}$. In other words, one considers a slab geometry with two infinite parallel hyperplanes separated by a distance $L_{d}$, or because of the periodic 
boundary conditions, a scalar field on the spatial manifold $\mathbb{R}^{d-1} \times \mathbb{S}_{L_{d}}^{1}$. Suppose also that one uses symmetric instead of normal ordering, so that

$$
\hat{H}=\frac{1}{2} \sum_{n_{i} \in \mathbb{Z}^{d}}^{\prime} \omega_{k_{i}}\left(\hat{a}_{k_{i}}^{\dagger} \hat{a}_{k_{i}}+\hat{a}_{k_{i}} \hat{a}_{k_{i}}^{\dagger}\right)=\sum_{n_{i} \in \mathbb{Z}^{d}}^{\prime} \omega_{k_{i}}\left(\hat{a}_{k_{i}}^{\dagger} \hat{a}_{k_{i}}+\frac{1}{2}\right) .
$$

The divergent zero-point energy is

$$
E_{0}^{d}=\langle 0|\hat{H}| 0\rangle=\frac{1}{2} \sum_{n_{i} \in \mathbb{Z}^{d}}^{\prime} \omega_{k_{i}} .
$$

Transforming $d-1$ sums into integrals, and using $\zeta$ function regularization to give a meaning to this divergent quantity, we need to evaluate

$$
E_{0}^{d}(s)=\frac{1}{2} \nu^{2 s} \frac{V_{d-1}}{(2 \pi)^{d-1}} \sum_{n_{d} \in \mathbb{Z}} \int \mathrm{d}^{d-1} k\left[\left(\frac{2 \pi n_{d}}{L_{d}}\right)^{2}+k_{I} k^{I}\right]^{-s+\frac{1}{2}},
$$

where $V_{d-1}=\prod_{I=1}^{d-1} L_{I}$. This is the same computation as in (2.34), up an overall factor of $\left(2 \pi \nu^{2}\right)^{s}$ versus $\frac{1}{2} \nu^{2 s}$ and the replacements $d \rightarrow d-1, s \rightarrow s-\frac{1}{2}, \beta=L_{d+1} \rightarrow L_{d}$. The result can thus be read off (2.39),

$$
E_{0}^{d}(s)=\frac{1}{2} \nu^{2 s} \frac{V_{d-1}}{L_{d}^{d-2 s}} 2^{2-2 s} \pi^{\frac{d+1}{2}-2 s} \frac{\Gamma\left(s-\frac{d}{2}\right) \zeta(2 s-d)}{\Gamma\left(s-\frac{1}{2}\right)},
$$

which yields at $s=0$,

$$
E_{0}^{d}(0)=-\xi(-d) \frac{V_{d-1}}{L_{d}^{d}}
$$

and thus, after using the reflection formula,

$$
E_{0}^{d}(0)=-\xi(d+1) \frac{V_{d-1}}{L_{d}^{d}}=\left\{\begin{array}{cc}
-\frac{\pi}{6} \frac{1}{L_{1}} & \text { for } d=1 \\
-\frac{\zeta(3)}{2 \pi} \frac{L_{1}}{L_{2}^{2}} & \text { for } d=2 \\
-\frac{\pi^{2}}{90} \frac{L_{1} L_{2}}{L_{3}^{3}} & \text { for } d=3 \\
\vdots
\end{array}\right.
$$

\section{Remarks}

(i) For later purpose and to make contact with the literature, one may write

$$
E_{0}^{d}=-\varepsilon_{\mathrm{vac}}^{d} \frac{V_{d-1}}{L_{d}^{d}}
$$

where $\varepsilon_{\text {vac }}^{d}$ is a dimensionless number characterizing the Casimir energy whose value is

$$
\varepsilon_{\mathrm{vac}}^{d}=\xi(d+1)
$$

in the current model.

The relation between the partition function (2.24) in the large volume limit and the Casimir energy density (see e.g. [6, 26-28, 40, 41]) may then be written as

$$
\ln Z_{d}(\beta)=\varepsilon_{\mathrm{vac}}^{d} \frac{V_{d}}{\beta^{d}} .
$$


(ii) On account of the previously discussed equivalence, the correct electromagnetic results with perfectly conducting plates at $x^{3}=0$ and $x^{3}=a$ are again obtained through the replacement $L_{3}=2 a$.

(iii) Note that, even with symmetric ordering,

$$
P_{i}^{0}=\left\langle 0\left|\hat{P}_{i}\right| 0\right\rangle=0,
$$

irrespective of the existence or not of a small spatial dimension, because either $\sum_{n_{i} \in \mathbb{Z}} k_{i}=0$ or $\int \mathrm{d} k_{i} k_{i}=0$.

(iv) The computation of the Casimir energy can also be done using a suitable integral representation. Starting from the representation (2.65) at $s=-\frac{1}{2}$ (to be understood in the sense of analytic continuation), the zero point energy in (2.73) is written as

$$
E_{0}^{d}=\frac{1}{2 \Gamma\left(-\frac{1}{2}\right)} \sum_{n_{i} \in \mathbb{Z}^{d}}^{\prime} \int_{0}^{\infty} \mathrm{d} t t^{-\frac{3}{2}} e^{-t \omega_{k_{i}}^{2}}
$$

After changing sums to integrals for the $d-1$ large dimensions and integrating over the angles, one gets

$$
E_{0}^{d}=-\frac{1}{4 \sqrt{\pi}} \frac{V_{d-1}}{(2 \pi)^{d-1}} \frac{2 \pi^{\frac{d-1}{2}}}{\Gamma\left(\frac{d-1}{2}\right)} \sum_{n_{d} \in \mathbb{Z}} \int_{0}^{\infty} \mathrm{d} t t^{-\frac{3}{2}} \int_{0}^{\infty} \mathrm{d} k k^{d-2} e^{-t\left[\left(\frac{2 \pi}{L_{d}} n_{d}\right)^{2}+k^{2}\right]} .
$$

The change of variables $k=\frac{2 \pi}{L_{d}} \sqrt{y}, t \rightarrow t=\left(\frac{L_{d}}{2 \pi}\right)^{2} t$ then leads to

$$
E_{0}^{d}=-\frac{V_{d-1}}{L_{d}^{d}} \frac{\pi^{\frac{d}{2}}}{2 \Gamma\left(\frac{d-1}{2}\right)} \sum_{n_{d} \in \mathbb{Z}} \int_{0}^{\infty} \mathrm{d} t t^{-\frac{3}{2}} e^{-t n_{d}^{2}} \int_{0}^{\infty} \mathrm{d} y y^{\frac{d-1}{2}-1} e^{-t y} .
$$

Performing the integral over $y$ using (2.65) again gives

$$
E_{0}^{d}=-\frac{V_{d-1}}{L_{d}^{d}} \frac{\pi^{\frac{d}{2}}}{2} \sum_{n_{d} \in \mathbb{Z}} \int_{0}^{\infty} \mathrm{d} t t^{-\frac{d}{2}-1} e^{-t n_{d}^{2}}
$$

The integral term with $n_{d}=0$ is divergent, $\int_{\epsilon}^{\infty} \mathrm{d} t t^{-\frac{d}{2}-1}=\frac{2 \epsilon^{-\frac{d}{2}}}{d}$ and neglected, while the terms with $n_{d} \neq 0$ are evaluated using (2.65) to yield

$$
E_{0}^{d}=-\frac{V_{d-1}}{L_{d}^{d}} \pi^{\frac{d}{2}} \Gamma\left(-\frac{d}{2}\right) \zeta(-d)
$$

in agreement with (2.76).

\section{Two small spacetime dimensions and Eisenstein series}

\subsection{Functional approach}

We now consider the case where only the first $d-1$ spatial dimensions are large, while $L_{d}$ is small, and where a chemical potential for linear momentum in the small dimension $x^{d}$ is 
turned on. We start here with the functional approach because it yields directly the result in terms of the Eisenstein series in (1.1). In this case $I=1, \ldots, d-1, V_{d-1}=\prod_{I=1}^{d-1} L_{I}$ and, when taking into account (2.30), we get instead of (2.34),

$$
\zeta_{-\Delta_{\mu}}(s)=\frac{V_{d-1}}{(2 \pi)^{d-1}} \int \mathrm{d}^{d-1} k \sum_{\left(n_{d+1}, n_{d}\right) \in \mathbb{Z}^{2}}^{\prime}\left[\left(\frac{2 \pi n_{d+1}}{\beta}-\mu \frac{2 \pi n_{d}}{L_{d}}\right)^{2}+\left(\frac{2 \pi n_{d}}{L_{d}}\right)^{2}+k_{I} k^{I}\right]^{-s} .
$$

The computation then proceeds as in section 2.4, up to the replacements $d \rightarrow d-1$ and of the zeta function by a suitable Eisenstein series. More precisely, instead of (2.37), we now get

$$
\begin{aligned}
& \zeta_{-\Delta_{\mu}}(s)=\frac{V_{d-1}}{(2 \pi)^{d-1}} \frac{2 \pi^{\frac{d-1}{2}}}{\Gamma\left(\frac{d-1}{2}\right)}\left(\frac{2 \pi}{\beta}\right)^{d-1-2 s} \\
& \sum_{\left(n_{d+1}, n_{d}\right) \in \mathbb{Z}^{2}}^{\prime} \int_{0}^{\infty} \mathrm{d} y y^{d-2}\left[\left(n_{d+1}-n_{d} \frac{\beta \mu}{L_{d}}\right)^{2}+n_{d}^{2}\left(\frac{\beta}{L_{d}}\right)^{2}+y^{2}\right]^{-s} .
\end{aligned}
$$

For later convenience, we also change parametrization and use $\alpha=\beta \mu$ so that we are now computing

$$
\mathrm{Z}_{d, 1}(\beta, \alpha)=\operatorname{Tr} e^{-\beta \hat{H}+i \alpha \hat{P}_{d}} .
$$

The additional subscript 1 in $Z_{d, 1}$ refers to the number of small spatial dimensions. In section 4 below, we will consider $q$ small spatial dimensions and compute $\mathrm{Z}_{d, q}$. Introducing the modular parameter

$$
\tau=\frac{\alpha+i \beta}{L_{d}}
$$

the sum simplifies to

$$
\sum_{\left(n_{d+1}, n_{d}\right) \in \mathbb{Z}^{2}}^{\prime} \int_{0}^{\infty} \mathrm{d} y y^{d-2}\left[\left|n_{d+1}+n_{d} \tau\right|^{2}+y^{2}\right]^{-s} .
$$

The integral is convergent for $\mathfrak{R e}(s)>\frac{d-1}{2}$ and can be performed through the change of variables $y=\left|n_{d+1}+n_{d} \tau\right|^{2} \sinh x$ which gives

$$
\zeta_{-\Delta_{\alpha}}(s)=\frac{V_{d-1}}{(2 \pi)^{d-1}} \frac{\pi^{\frac{d-1}{2}} \Gamma\left(s-\frac{d-1}{2}\right)}{\Gamma(s)}\left(\frac{2 \pi}{\beta}\right)^{d-1-2 s} \sum_{\left(n_{d+1}, n_{d}\right) \in \mathbb{Z}^{2}}^{\prime}\left|n_{d+1}+n_{d} \tau\right|^{d-1-2 s} .
$$

In terms of the real analytic Eisenstein series (1.1), this can be written as

$$
\zeta_{-\Delta_{\alpha}}(s)=\frac{V_{d-1}}{(2 \pi)^{d-1}} \frac{\pi^{\frac{d-1}{2}} \Gamma\left(s-\frac{d-1}{2}\right)}{\Gamma(s) \tau_{2}^{s-\frac{d-1}{2}}}\left(\frac{2 \pi}{\beta}\right)^{d-1-2 s} f_{s-\frac{d-1}{2}}(\tau, \bar{\tau}) .
$$

When using the functional relation for the analytically continued Eisenstein series,

$$
\pi^{-z} \Gamma(z) f_{z}(\tau, \bar{\tau})=\pi^{z-1} \Gamma(1-z) f_{1-z}(\tau, \bar{\tau}),
$$


for $z=s-\frac{d-1}{2}$, and also $\tau_{2}=\frac{\beta}{L_{d}}$, we end up with

$$
\zeta_{-\Delta_{\alpha}}(s)=\frac{\Gamma\left(\frac{d+1}{2}-s\right)}{2^{2 s} \pi^{\frac{d+1}{2}} \Gamma(s)} \frac{V_{d-1}}{L_{d}^{d-1-2 s}} \frac{f_{\frac{d+1}{2}-s}(\tau, \bar{\tau})}{\tau_{2}^{\frac{d-1}{2}-s}} .
$$

Again, since $\Gamma(s) \approx \frac{1}{s}+O\left(s^{0}\right), \zeta_{-\Delta_{\alpha}}(0)$, if $\mathcal{Z}_{d, 1}(\tau, \bar{\tau})=\mathrm{Z}_{d, 1}(\beta, \alpha)$, the partition function is

$$
\ln \mathcal{Z}_{d, 1}(\tau, \bar{\tau})=\frac{\Gamma\left(\frac{d+1}{2}\right) V_{d-1}}{2 \pi^{\frac{d+1}{2}} L_{d}^{d-1} \tau_{2}^{\frac{d-1}{2}}} f_{\frac{d+1}{2}}(\tau, \bar{\tau}) .
$$

In terms of the completion of the real analytic Eisenstein series,

$$
\xi(z ; \tau, \bar{\tau})=\frac{\Gamma\left(\frac{z}{2}\right) f_{\frac{z}{2}}(\tau, \bar{\tau})}{2 \pi^{\frac{z}{2}}},
$$

which satisfies the reflection formula

$$
\xi(z ; \tau, \bar{\tau})=\xi(2-z ; \tau, \bar{\tau})
$$

this gives

$$
\ln \mathcal{Z}_{d, 1}(\tau, \bar{\tau})=\frac{V_{d-1}}{L_{d}^{d-1} \tau_{2}^{\frac{d-1}{2}}} \xi(d+1 ; \tau, \bar{\tau}) .
$$

Since $\xi(z ; \tau, \bar{\tau})$ is invariant under modular transformations (1.2) while $\tau_{2}$ transforms as

$$
\tau_{2} \rightarrow \frac{\tau_{2}}{|c \tau+d|^{2}}
$$

the partition function (3.11) transforms as

$$
\ln \mathcal{Z}_{d, 1}\left(\tau^{\prime}, \bar{\tau}^{\prime}\right)=|c \tau+d|^{d-1} \ln \mathcal{Z}_{d, 1}(\tau, \bar{\tau}) \text {. }
$$

\section{Remarks}

(i) The above result holds for $d>1$ because if $d=1$, i.e., for the free boson on $\mathbb{T}^{2}$, $\ln \mathcal{Z}_{1,1}(\tau, \bar{\tau})=\frac{1}{2 \pi} f_{1}(\tau, \bar{\tau})$, is not convergent and more care is needed to derive the correct result. This is reviewed in section 3.4.

(ii) As discussed in the introduction, for $d=3$, the result is directly relevant for the electromagnetic field with Casimir boundary conditions after the replacement $L_{3}=2 a$.

(iii) If the chemical potential vanishes, $\alpha=0, \tau=i \tau_{2}$, with $\tau_{2}=\frac{\beta}{L_{d}}$ and $\mathcal{Z}_{d, 1}\left(i \tau_{2},-i \tau_{2}\right)=$ $\mathcal{Z}_{d, 1}\left(\tau_{2}\right)=Z_{d, 1}(\beta)$, the result can be written in terms of an Epstein zeta function,

$$
\zeta\left(s ; 1, \tau_{2}^{2}\right)=\sum_{\left(m^{d+1}, m^{d}\right) \in \mathbb{Z}^{2}}^{\prime} \frac{1}{\left[\left(m^{d}\right)^{2}+\left(m^{d+1}\right)^{2} \tau_{2}^{2}\right]^{s}},
$$

as

$$
\ln \mathcal{Z}_{d, 1}\left(\tau_{2}\right)=\frac{\Gamma\left(\frac{d+1}{2}\right)}{2 \pi^{\frac{d+1}{2}}} \frac{V_{d-1} \tau_{2}}{L_{d}^{d-1}} \zeta\left(\frac{d+1}{2} ; 1, \tau_{2}^{2}\right)
$$


If $a=0=d, b=1=-c$, the modular transformation reduces to temperature inversion $\tau_{2} \rightarrow \frac{1}{\tau_{2}}$ with

$$
\ln \mathcal{Z}_{d, 1}\left(\frac{1}{\tau_{2}}\right)=\tau_{2}^{d-1} \ln \mathcal{Z}_{d, 1}\left(\tau_{2}\right)
$$

Some properties and a trigonometric expansion for the Epstein zeta function are reviewed in appendix B.

(iv) Setting $m^{d}=0$ in $(3.17)$ gives

$$
\ln \mathcal{Z}_{d, 1}^{\text {high }}\left(\tau_{2}\right)=\xi(d+1) \frac{V_{d-1}}{L_{d}^{d-1} \tau_{2}^{d}}
$$

which is the black body result (2.24). From the analysis of section 2, we know that this is the dominant contribution in the high temperature/large $L_{d}$ limit $\tau_{2} \ll 1$. This justifies a posteriori the use of a normal ordered Hamiltonian in section 2.2.

(v) From equation (3.18), it follows that in the low temperature/small $L_{d}$ limit $\tau_{2} \gg 1$, the dominant contribution is given by

$$
\ln \mathcal{Z}_{d, 1}^{\text {low }}\left(\tau_{2}\right)=\xi(d+1) \frac{V_{d-1} \tau_{2}}{L_{d}^{d-1}} .
$$

The result for the Casimir energy with one small spatial dimension in (2.77), then follows by taking $-\frac{\partial}{\partial \beta}$ of this dominant contribution.

(vi) Without necessarily implying the existence of an underlying infinite-dimensional Virasoro algebra, one may write the partition function in a form suggested by conformal field theories in 2 spacetime dimensions as

$$
\mathcal{Z}_{d, 1}(\tau, \bar{\tau})=\operatorname{Tr} e^{2 \pi i \tau\left(\hat{L}_{0}-\frac{c_{d}}{24}\right)-2 \pi i \bar{\tau}\left(\hat{\bar{L}}_{0}-\frac{c_{d}}{24}\right)} .
$$

When taking the original expression of the partition function (3.3) and the expression of the modular parameter (3.4) into account, it follows that

$$
\hat{L}_{0}=\frac{L_{d}}{4 \pi}\left(\hat{H}+\hat{P}_{d}\right)+\frac{c_{d}}{24}, \quad \hat{\bar{L}}_{0}=\frac{L_{d}}{4 \pi}\left(\hat{H}-\hat{P}_{d}\right)+\frac{c_{d}}{24},
$$

or, equivalently,

$$
\hat{H}=\frac{2 \pi}{L_{d}}\left(\hat{L}_{0}+\hat{\bar{L}}_{0}-\frac{c_{d}}{12}\right), \quad \hat{P}_{d}=\frac{2 \pi}{L_{d}}\left(\hat{L}_{0}-\hat{\bar{L}}_{0}\right) .
$$

Note that in terms of the energy-momentum tensor,

$$
T_{\mu \nu}=\partial_{\mu} \phi \partial_{\nu} \phi-\frac{1}{2} \eta_{\mu \nu} \partial_{\rho} \phi \partial^{\rho} \phi
$$

these observables are given by

$$
L_{0}=\frac{L_{d}}{2 \pi} \int_{V_{d}} \mathrm{~d}^{d} x\left(T_{--}+T_{+-}\right), \quad \bar{L}_{0}=\frac{L_{d}}{2 \pi} \int_{V_{d}} \mathrm{~d}^{d} x\left(T_{++}+T_{+-}\right),
$$


where $x^{ \pm}=x^{0} \pm x^{d}, T_{ \pm \pm}=\partial_{ \pm} \phi \partial_{ \pm} \phi$ and $T_{+-}=\frac{1}{4} \partial_{I} \phi \partial^{I} \phi$ no longer vanishes for $d>1$. As follows from Remark (v) and will be worked out in detail in section 3.2, in a low temperature expansion, the leading contribution to the partition function is

$$
\ln \mathrm{Z}_{d, 1}^{\text {low }}(\beta, 0)=-\beta E_{0}^{d},
$$

with $E_{0}^{d}$ given in (2.77). Identifying this contribution with that involving the central charge $c_{d}$ in (3.21), it follows that

$$
E_{0}^{d}=-\frac{\pi c_{d}}{6 L_{d}}
$$

and then, when taking (2.77) into account, that

$$
c_{d}=\frac{6 \xi(d+1)}{\pi} \frac{V_{d-1}}{L_{d}^{d-1}} .
$$

In terms of the central charge, the partition function becomes

$$
\ln \mathcal{Z}_{d, 1}(\tau, \bar{\tau})=\frac{\pi c_{d}}{12 \zeta(d+1)} \tau_{2}^{-\frac{d-1}{2}} f_{\frac{d+1}{2}}(\tau, \bar{\tau})
$$

while

$$
\varepsilon_{\mathrm{vac}}^{d}=\frac{\pi c_{d}}{6} \frac{L_{d}^{d}}{V_{d}}
$$

It also follows from Remark (iv) and will be worked out in detail in section 3.2, that the leading contribution at high temperature/ large $L_{d}$ is

$$
\ln \mathrm{Z}_{d, 1}^{\text {high }}(\beta, 0)=\frac{\pi c_{d} L_{d}^{d}}{6 \beta^{d}} .
$$

Following [18], the associated asymptotic micro-canonical density of states is then

$$
\rho_{d}(E)=\int_{0}^{\infty} \mathrm{d} \beta Z_{d, 1}^{\text {high }}(\beta, 0) e^{\beta E}=\int_{0}^{\beta} \mathrm{d} \beta e^{\frac{\pi c_{d} L_{d}^{d}}{6 \beta^{d}}+\beta E},
$$

which yields in the saddle point approximation,

$$
\ln \rho_{d}(E) \approx(d+1)\left(\frac{E L_{d}}{d}\right)^{\frac{d}{d+1}}\left(\frac{\pi c_{d}}{6}\right)^{\frac{1}{d+1}} .
$$

(vii) Real analytic Eisenstein series are eigenfunctions of the Laplace-Beltrami operator on the upper half-plane $\mathbb{H}$ (see e.g. [4]),

$$
\Delta_{\mathbb{H}}=\tau_{2}^{2}\left(\partial_{\tau_{1}}^{2}+\partial_{\tau_{2}}^{2}\right), \quad \Delta_{\mathbb{H}} f_{s}(\tau, \bar{\tau})=s(s-1) f_{s}(\tau, \bar{\tau}) .
$$

In terms of $\ln \mathcal{Z}_{d, 1}(\tau, \bar{\tau})$, one finds

$$
\Delta_{\mathbb{H}} \ln \mathcal{Z}_{d, 1}=(d-1)\left[\ln \mathcal{Z}_{d, 1}-\tau_{2} \partial_{\tau_{2}} \ln \mathcal{Z}_{d, 1}\right] .
$$


When taking into account that

$$
\langle\hat{H}\rangle=-\partial_{\beta} \ln \mathrm{Z}_{d, 1}(\beta, \alpha), \quad\left\langle\hat{P}_{d}\right\rangle=-i \partial_{\alpha} \ln \mathrm{Z}_{d, 1}(\beta, \alpha),
$$

as well as

$$
\Delta \hat{O}=\hat{O}-\langle\hat{O}\rangle, \quad\left\langle(\Delta \hat{O})^{2}\right\rangle=\left\langle\hat{O}^{2}\right\rangle-(\langle\hat{O}\rangle)^{2},
$$

the interpretation of equation (3.35) in quantum statistical terms is

$$
\beta^{2}\left[\left\langle(\Delta \hat{H})^{2}-\left(\Delta \hat{P}_{d}\right)^{2}\right\rangle\right]=(d-1)\left[\ln \mathrm{Z}_{d, 1}+\beta\langle\hat{H}\rangle\right] .
$$

Alternatively, using $\Delta_{\mathbb{H}}=-(\tau-\bar{\tau})^{2} \partial_{\tau} \partial_{\bar{\tau}}$, this may be written as

$$
\begin{aligned}
&-(2 \pi(\tau-\bar{\tau}))^{2}\left[\left\langle\hat{L}_{0} \hat{\bar{L}}_{0}\right\rangle-\left\langle\hat{L}_{0}\right\rangle\left\langle\hat{\bar{L}}_{0}\right\rangle\right] \\
&=(d-1)\left[\ln \mathcal{Z}_{d, 1}-i \pi(\tau-\bar{\tau})\left\langle\hat{L}_{0}+\hat{\bar{L}}_{0}-\frac{c_{d}}{12}\right\rangle\right] .
\end{aligned}
$$

(viii) As in section 2.7, the regularized Casimir energy on the spatial $\mathbb{R}^{d-2} \times \mathbb{T}^{2}$, where $\mathbb{T}^{2}$ is rectangular, can be obtained from the zeta function in (3.9), by multiplying the result by $\frac{1}{2} \nu^{2 s}$ and the replacements $\tau \rightarrow i \frac{L_{d}}{L_{d-1}}, d \rightarrow d-1, s \rightarrow s-\frac{1}{2}$. If $x^{I}$, for $I=1 \ldots d-2$, denote the large dimensions and $V_{d-2}=\prod_{I=1}^{d-2} L_{I}$,

$$
E_{0}(s)=\frac{1}{2} \nu^{2 s} \frac{\Gamma\left(\frac{d+1}{2}-s\right)}{2^{2 s-1} \pi^{\frac{d}{2}} \Gamma\left(s-\frac{1}{2}\right)} \frac{V_{d-2} L_{d}}{L_{d-1}^{d-2 s}} \zeta\left(\frac{d+1}{2}-s ;\left(\frac{L_{d}}{L_{d-1}}\right)^{2}, 1\right),
$$

so that

$$
E_{0}(0)=-\frac{1}{2} \frac{\Gamma\left(\frac{d+1}{2}\right)}{\pi^{\frac{d+1}{2}}} \frac{V_{d-2} L_{d}}{L_{d-1}^{d}} \zeta\left(\frac{d+1}{2} ;\left(\frac{L_{d}}{L_{d-1}}\right)^{2}, 1\right)
$$

\subsection{Canonical approach}

In this section, we evaluate the partition function

$$
\mathrm{Z}_{d, 1}(\beta, \alpha)=\operatorname{Tr} e^{-\beta \hat{H}+i \alpha \hat{P}_{d}},
$$

directly in the operator formalism where $\hat{H}$ and $\hat{P}_{d}$ are given by

$$
\hat{H}=\sum_{n_{i} \in \mathbb{Z}^{d}} \omega_{k} \hat{a}_{k_{i}}^{\dagger} \hat{a}_{k_{i}}+E_{0}^{d}, \quad \hat{P}_{d}=\sum_{n_{i} \in \mathbb{Z}^{d}} k_{d} \hat{a}_{k_{i}}^{\dagger} \hat{a}_{k_{i}}
$$

and $E_{0}^{d}$ is given in (2.77). It follows that

$$
\mathrm{Z}_{d, 1}(\beta, \alpha)=e^{-\beta E_{0}^{d}} \prod_{n_{i} \in \mathbb{Z}^{d}}^{\prime} \sum_{N_{k_{i}} \in \mathbb{N}} e^{\left(-\beta \omega_{k}+i \alpha k_{d}\right) N_{k_{i}}}=e^{-\beta E_{0}^{d}} \prod_{n_{i} \in \mathbb{Z}^{d}}^{\prime} \frac{1}{1-e^{-\beta \omega_{k}+i \alpha k_{d}}} .
$$


Turning again the sums into integrals in the large dimensions gives

$$
\begin{aligned}
\ln \mathrm{Z}_{d, 1}(\beta, \alpha) & =-\beta E_{0}^{d}-\frac{V_{d-1}}{(2 \pi)^{d-1}} \int_{-\infty}^{\infty} \mathrm{d}^{d-1} k \sum_{n_{d} \in \mathbb{Z}} \ln \left[1-e^{-\beta \omega_{k}+i \alpha k_{d}}\right] \\
& =-\beta E_{0}^{d}-\frac{V_{d-1}}{(2 \pi)^{d-1}} \frac{2 \pi^{\frac{d-1}{2}}}{\Gamma\left(\frac{d-1}{2}\right)} \sum_{n_{d} \in \mathbb{Z}} \int_{0}^{\infty} \mathrm{d} k k^{d-2} \ln \left[1-e^{-\beta \sqrt{k^{2}+k_{d}^{2}}+i \alpha k_{d}}\right],
\end{aligned}
$$

where $k=k_{I} k^{I}$, the range of $I$ is from 1 to $d-1$ and $V_{d-1}=\prod_{I=1}^{d-1} L_{I}$. For the sum over $n_{d}$, it is convenient to split $n_{d}=0$ from the other terms. When using (2.20) for $d \rightarrow d-1$ for the former term, we get

$$
\ln \mathrm{Z}_{d, 1}(\beta, \alpha)=-\beta E_{0}^{d}+\frac{\Gamma(d-1) \zeta(d)}{2^{d-2} \pi^{\frac{d-1}{2}} \Gamma\left(\frac{d-1}{2}\right)} \frac{V_{d-1}}{\beta^{d-1}}-\frac{V_{d-1}}{2^{d-2} \pi^{\frac{d-1}{2}} \Gamma\left(\frac{d-1}{2}\right)} \sum_{n_{d} \in \mathbb{Z}}^{\prime} I_{d}\left(\beta, \alpha ; n_{d}\right),
$$

where

$$
I_{d}\left(\beta, \alpha ; n_{d}\right)=\int_{0}^{\infty} \mathrm{d} k k^{d-2} \ln \left[1-e^{-\beta \sqrt{k^{2}+k_{d}^{2}}+i \alpha k_{d}}\right] .
$$

Introducing the variable $z$ as

$$
z=\beta \sqrt{k^{2}+k_{d}^{2}}, \quad k^{d-2} \mathrm{~d} k=\frac{1}{\beta^{2} \beta^{d-3}}\left(z^{2}-\beta^{2} k_{d}^{2}\right)^{\frac{d-3}{2}} z \mathrm{~d} z,
$$

we get

$$
I_{d}\left(\beta, \alpha ; n_{d}\right)=\frac{1}{\beta^{d-1}} \int_{\beta\left|k_{d}\right|}^{\infty} \mathrm{d} z z\left(z^{2}-\beta^{2} k_{d}^{2}\right)^{\frac{d-3}{2}} \ln \left[1-e^{-z+i \alpha k_{d}}\right] .
$$

Expanding the logarithm as

$$
\ln \left[1-e^{-z+i \alpha k_{d}}\right]=-\sum_{l \in \mathbb{N}^{*}} \frac{e^{-l z+i l \alpha k_{d}}}{l},
$$

we get, after the change of variables $z^{\prime}=l z$,

$$
I_{d}\left(\beta, \alpha ; n_{d}\right)=-\frac{1}{\beta^{d-1}} \sum_{l \in \mathbb{N}^{*}} \frac{e^{i l \alpha k_{d}}}{l^{d}} \int_{l \beta\left|k_{d}\right|}^{\infty} \mathrm{d} z z\left(z^{2}-l^{2} \beta^{2} k_{d}^{2}\right)^{\frac{d-3}{2}} e^{-z} .
$$

The integrals are given in terms of a modified Bessel function of the second kind as

$$
\int_{l \beta\left|k_{d}\right|}^{\infty} \mathrm{d} z z\left(z^{2}-l^{2} \beta^{2} k_{d}^{2}\right)^{\frac{d-3}{2}} e^{-z}=2^{\frac{d-2}{2}} \frac{\Gamma\left(\frac{d-1}{2}\right)}{\sqrt{\pi}}\left(l \beta\left|k_{d}\right|\right)^{\frac{d}{2}} K_{\frac{d}{2}}\left(l \beta\left|k_{d}\right|\right),
$$

so that

$$
\begin{aligned}
\ln \mathrm{Z}_{d, 1}(\beta, \alpha)= & -\beta E_{0}^{d}+\frac{\Gamma(d-1) \zeta(d)}{2^{d-2} \pi^{\frac{d-1}{2}} \Gamma\left(\frac{d-1}{2}\right)} \frac{V_{d-1}}{\beta^{d-1}} \\
& +2 \frac{V_{d-1}}{L_{d}^{\frac{d}{2}} \beta^{\frac{d-2}{2}}} \sum_{n_{d} \in \mathbb{Z}}^{\prime} \sum_{l \in \mathbb{N}^{*}}\left(\frac{\left|n_{d}\right|}{l}\right)^{\frac{d}{2}} K_{\frac{d}{2}}\left(2 \pi l\left|n_{d}\right| \frac{\beta}{L_{d}}\right) e^{2 \pi i l n_{d} \frac{\alpha}{L_{d}}} .
\end{aligned}
$$




\section{Remarks}

(i) At low temperature/small distance $\frac{\beta}{L_{d}} \gg 1$, the leading term in the expansion of the partition function is directly related to the Casimir energy. The leading correction is the contribution of the modes with spatial frequencies $n_{d}=0$. It coincides with the black body result (2.24) of a massless scalar field in $d-1$ spatial dimensions. On account of the equivalence of this expression with (2.82), it can also be written more compactly as $\xi(d) \frac{V_{d-1}}{\beta^{d-1}}$. It is independent of $L_{d}$ and thus does not contribute to the Casimir pressure,

$$
\mathrm{p}_{d, 1}(\beta, \alpha)=\frac{1}{V_{d-1}} \frac{\partial\left(\beta^{-1} \ln \mathrm{Z}_{d, 1}(\beta, \alpha)\right)}{\partial L_{d}} .
$$

The asymptotic expansion

$$
K_{\nu}(x)=\sqrt{\frac{\pi}{2 x}} e^{-x}\left(1+O\left(x^{-1}\right)\right),
$$

for large $x$, implies that all other terms are exponentially suppressed. It follows that low-temperature/small distance expansion of the Casimir pressure is

$$
\mathrm{p}_{d, 1}^{\text {low }}(\beta, \alpha)=-d \xi(d+1) \frac{1}{L_{d}^{d+1}}+\ldots,
$$

where the dots denote exponentially suppressed terms. In the low-temperature/small distance expansion of the entropy,

$$
\mathrm{S}_{d, 1}(\beta, \alpha)=\left(1-\beta \partial_{\beta}\right) \ln \mathrm{Z}_{d, 1}(\beta, \alpha),
$$

on the other hand, the first term in (3.53) proportional to the Casimir energy in $d$ spatial dimensions drops out since it is linear in $\beta$ and the leading term now comes from the lower dimensional scalar field, i.e., the modes with $n_{d}=0$,

$$
\mathrm{S}_{d, 1}^{\mathrm{low}}(\beta, \alpha)=d \xi(d) \frac{V_{d-1}}{\beta^{d-1}}+\ldots \text {. }
$$

It may be written in terms of the dimensionless number characterizing the Casimir energy density defined in (2.78) in $d-1$ spatial dimensions as

$$
\mathrm{S}_{d, 1}^{\text {low }}(\beta, \alpha)=d \varepsilon_{\mathrm{vac}}^{d-1} \frac{V_{d-1}}{\beta^{d-1}}+\ldots
$$

This is a novel low-temperature formula for the model under consideration, that relates the leading contribution to the entropy to the Casimir energy density of a massless scalar field in one dimension lower. Even though it looks similar, it is not the higher dimensional generalization of the Cardy formula $[14,16]$ discussed in [18], which is valid at high temperature (see below). Since the two leading terms in the low temperature/small intervall expansion of (3.53) do not depend on $\alpha$ they are the same when starting from $\ln Z_{d, 1}(\beta, \mu)$. It follows that the leading terms in the expansion of

$$
p_{d, 1}(\beta, \mu)=\frac{1}{V_{d-1}} \frac{\partial\left(\beta^{-1} \ln Z_{d, 1}(\beta, \mu)\right)}{\partial L_{d}}, \quad S_{d, 1}(\beta, \mu)=\left(1-\beta \partial_{\beta}\right) \ln Z_{d, 1}(\beta, \mu),
$$

are still given by the right hand sides of (3.56) and of (3.58). 
(ii) When changing the double sum in the last term to a sum over $m=\ln _{d} \in \mathbb{Z}^{*}$ and introducing the divisor sum

$$
\sigma_{s}(m)=\sum_{n \mid m} n^{s}
$$

the exponentially suppressed terms, i.e., the last line of (3.53), may be written as

$$
2 \frac{V_{d-1}}{L_{d}^{\frac{d}{2}} \beta^{\frac{d-2}{2}}} \sum_{m \in \mathbb{Z}}^{\prime} \sigma_{-d}(m)|m|^{\frac{d}{2}} K_{\frac{d}{2}}\left(2 \pi m \frac{\beta}{L_{d}}\right) e^{2 \pi i m \frac{\alpha}{L_{d}}}
$$

When taking into account the explicit expression for the vacuum energy $E_{0}^{d}$ in (2.77), the partition function may be re-written as

$$
\begin{aligned}
& \ln \mathrm{Z}_{d, 1}(\beta, \alpha)=\frac{V_{d-1}\left[\xi(d+1) \frac{\beta}{L_{d}}+\xi(d)\left(\frac{L_{d}}{\beta}\right)^{d-1}\right.}{\left.+2\left(\frac{L_{d}}{\beta}\right)^{\frac{d-2}{2}} \sum_{m \in \mathbb{Z}}^{\prime} \sigma_{-d}(m)|m|^{\frac{d}{2}} K_{\frac{d}{2}}\left(2 \pi m \frac{\beta}{L_{d}}\right) e^{\left.2 \pi i m \frac{\alpha}{L_{d}}\right]}\right]} \\
& +.
\end{aligned}
$$

The equivalence of the functional Lagrangian and the canonical approaches to computing the partition function then implies that this last result is the same than (3.10). For $s=\frac{d+1}{2}$, this shows

$$
\begin{aligned}
f_{s}(\tau, \bar{\tau})=2 \zeta(2 s) & {\left[\tau_{2}^{s}+\frac{\xi(2 s-1)}{\xi(2 s)} \tau_{2}^{1-s}\right.} \\
& \left.+\frac{2}{\xi(2 s)} \tau_{2}^{\frac{1}{2}} \sum_{m}^{\prime} \sigma_{1-2 s}(m)|m|^{\frac{2 s-1}{2}} K_{\frac{2 s-1}{2}}\left(2 \pi m \tau_{2}\right) e^{2 \pi i m \tau_{1}}\right]
\end{aligned}
$$

which is the Fourier expansion of $f_{s}(\tau, \bar{\tau})$, traditionally derived using Poisson resummation (see e.g. [4], appendix A).

(iii) When expressed in terms of inverse temperature and chemical potential, the generating set of transformations of the modular group become

$$
\tau^{\prime}=\tau+1 \Longleftrightarrow\left\{\begin{array}{l}
\left(\frac{\beta}{L_{d}}\right)^{\prime}=\frac{\beta}{L_{d}} \\
\left(\frac{\alpha}{L_{d}}\right)^{\prime}=\frac{\alpha}{L_{d}}+1
\end{array} \quad \tau^{\prime}=-\frac{1}{\tau} \Longleftrightarrow\left\{\begin{array}{l}
\left(\frac{\beta}{L_{d}}\right)^{\prime}=\frac{L_{d} \beta}{\alpha^{2}+\beta^{2}} \\
\left(\frac{\alpha}{L_{d}}\right)^{\prime}=-\frac{L_{d} \alpha}{\alpha^{2}+\beta^{2}}
\end{array} .\right.\right.
$$

Under the first of these transformations, $\ln \mathrm{Z}_{d, 1}(\beta, \alpha)$ in (3.63) is manifestly invariant, as required by (3.15) for $c=0, a=b=d=1$. For the second of these transformations, we get

$$
\ln \mathrm{Z}_{d, 1}\left(\beta^{\prime}, \alpha^{\prime}\right)=\frac{\left(\alpha^{2}+\beta^{2}\right)^{\frac{d-1}{2}}}{L_{d}^{d-1}} \ln \mathrm{Z}_{d, 1}(\beta, \alpha)
$$


When transposing and using the explicit expression in (3.63) for the l.h.s., this gives

$$
\begin{aligned}
& \ln \mathrm{Z}_{d, 1}(\beta, \alpha)=\frac{V_{d-1}}{\left(\alpha^{2}+\beta^{2}\right)^{\frac{d-1}{2}}}\left[\xi(d+1) \frac{L_{d} \beta}{\alpha^{2}+\beta^{2}}+\xi(d)\left(\frac{\alpha^{2}+\beta^{2}}{L_{d} \beta}\right)^{d-1}\right. \\
& \left.\quad+2\left(\frac{\alpha^{2}+\beta^{2}}{L_{d} \beta}\right)^{\frac{d-2}{2}} \sum_{m \in \mathbb{Z}}^{\prime} \sigma_{-d}(m)|m|^{\frac{d}{2}} K_{\frac{d}{2}}\left(2 \pi m \frac{L_{d} \beta}{\alpha^{2}+\beta^{2}}\right) e^{-2 \pi i m \frac{L_{d} \alpha}{\alpha^{2}+\beta^{2}}}\right] .
\end{aligned}
$$

For a high temperature/large interval expansion, it is more convenient to use $\mu$ rather than $\alpha$, in terms of which the previous expression becomes

$$
\begin{aligned}
& \ln Z_{d, 1}(\beta, \mu)=\frac{V_{d-1}}{L_{d}^{d-1}\left(1+\mu^{2}\right)^{\frac{d-1}{2}}}\left[\frac{\xi(d+1)}{1+\mu^{2}}\left(\frac{\beta}{L_{d}}\right)^{-d}+\xi(d)\left(1+\mu^{2}\right)^{d-1}+\right. \\
& \left.+2\left(\frac{\beta}{L_{d}}\right)^{-\frac{d}{2}}\left(1+\mu^{2}\right)^{\frac{d-2}{2}} \sum_{m \in \mathbb{Z}}^{\prime} \sigma_{-d}(m)|m|^{\frac{d}{2}} K_{\frac{d}{2}}\left(2 \pi m \frac{L_{d}}{\beta} \frac{1}{1+\mu^{2}}\right) e^{-2 \pi i m \frac{L_{d}}{\beta} \frac{1}{1+\mu^{2}}}\right] .
\end{aligned}
$$

The high temperature/large interval expansion is then defined by $\frac{\beta}{L_{d}} \ll 1$ at fixed $\mu$. The leading contributions are given by the first two terms, while the others are exponentially suppressed,

$$
\ln Z_{d, 1}^{\text {high }}(\beta, \mu)=\frac{V_{d-1} L_{d}}{\beta^{d}} \xi(d+1)\left(1+\mu^{2}\right)^{-\frac{d+1}{2}}+\frac{V_{d-1}}{L_{d}^{d-1}} \xi(d)\left(1+\mu^{2}\right)^{\frac{d-1}{2}}+\ldots
$$

Up to suitable powers of $\left(1+\mu^{2}\right)$, which will be explained in the general case below, the two leading terms in the expansion here are thus mapped to the two leading terms in the low temperature/small interval expansion (3.67) by an $S$ transformation $\tau \rightarrow-\frac{1}{\tau}$. The associated expansion of the Casimir pressure is

$$
p_{d, 1}^{\text {high }}(\beta, \mu)=\frac{\xi(d+1)}{\beta^{d+1}}\left(1+\mu^{2}\right)^{-\frac{d+1}{2}}-\frac{(d-1) \xi(d)}{\beta L_{d}^{d}}\left(1+\mu^{2}\right)^{\frac{d-1}{2}}+\ldots,
$$

while for the entropy, one finds

$$
S_{d, 1}^{\text {high }}(\beta, \mu)=\frac{V_{d-1} L_{d}}{\beta^{d}}(d+1) \xi(d+1)\left(1+\mu^{2}\right)^{-\frac{d+1}{2}}+\frac{V_{d-1}}{L_{d}^{d-1}} \xi(d)\left(1+\mu^{2}\right)^{\frac{d-1}{2}}+\ldots
$$

This can be written as

$$
S_{d, 1}^{\text {high }}(\beta, \mu)=(d+1) \varepsilon_{\text {vac }}^{d} \frac{V_{d}}{\beta^{d}}\left(1+\mu^{2}\right)^{-\frac{d+1}{2}}+\varepsilon_{\text {vac }}^{d-1} \frac{V_{d-1}}{L_{d}^{d-1}}\left(1+\mu^{2}\right)^{\frac{d-1}{2}}+\ldots
$$

The first term, which can also be written as,

$$
S_{d, 1}^{\text {high }}(\beta, \mu) \approx(d+1) \frac{\pi c_{d} L_{d}^{d}}{6 \beta^{d}}\left(1+\mu^{2}\right)^{-\frac{d+1}{2}},
$$

generalizes formula (8) of [18] to the case of a non-vanishing chemical potential. The associated asymptotic density of states becomes

$$
\ln \rho_{d}(E, \mu) \approx(d+1)\left(\frac{E L_{d}}{d}\right)^{\frac{d}{d+1}}\left(\frac{\pi c_{d}}{6}\right)^{\frac{1}{d+1}} \frac{1}{\sqrt{1+\mu^{2}}} .
$$




\subsection{Worldline approach}

Consider a particle on a torus with

$$
\hat{\mathrm{H}}_{\beta, \alpha}=\left(\hat{p}_{d+1}-\frac{\alpha}{\beta} \hat{p}_{d}\right)^{2}+\hat{p}_{d}^{2} .
$$

The associated heat kernel is

$$
\left\langle x^{\prime}\left|e^{-t \hat{\mathrm{H}}_{\beta, \alpha}}\right| x\right\rangle=\frac{1}{\beta L_{d}} \sum_{\left(n_{d+1}, n_{d}\right) \in \mathbb{Z}^{2}} e^{-t f},
$$

where

$$
f=\left|\frac{2 \pi n_{d+1}}{\beta}+\frac{2 \pi(\alpha+i \beta) n_{d}}{\beta L_{d}}\right|^{2}+i 2 \pi\left[n_{d+1} \frac{x_{d+1}^{\prime}-x_{d+1}}{\beta}+n_{d} \frac{x_{d}^{\prime}-x_{d}}{L_{d}}\right],
$$

which can again be shown by inserting a resolution of identity. When combining this heat kernel with the heat kernel (2.50) for $d-1$ large dimensions, one gets, after dropping the $n_{d+1}=0=n_{d}$ term,

$$
\ln \mathrm{Z}_{d, 1}(\beta, \alpha)=\frac{V_{d-1}}{2^{d} \pi^{\frac{d-1}{2}}} \sum_{\left(n_{d+1}, n_{d}\right) \in \mathbb{Z}^{2}}^{\prime} \int_{0}^{\infty} \mathrm{d} t t^{-\frac{d+1}{2}} e^{-t\left(\frac{2 \pi}{\beta}\right)^{2}\left|n_{d+1}+\tau n_{d}\right|^{2}} .
$$

Using again (2.65),

$$
\begin{aligned}
& \frac{1}{2^{d} \pi^{\frac{d-1}{2}}} \sum_{\left(n_{d+1}, n_{d}\right) \in \mathbb{Z}^{2}}^{\prime} \int_{0}^{\infty} \mathrm{d} t t^{-\frac{d+1}{2}} e^{-t\left(\frac{2 \pi}{\beta}\right)^{2}\left|n_{d+1}+\tau n_{d}\right|^{2}} \\
& =\frac{\Gamma\left(\frac{1-d}{2}\right)}{2^{d} \pi^{\frac{d-1}{2}}}\left(\frac{2 \pi}{\beta}\right)^{d-1} \sum_{\left(n_{d+1}, n_{d}\right) \in \mathbb{Z}^{2}}^{\prime} \frac{1}{\left|n_{d+1}+\tau n_{d}\right|^{1-d}},
\end{aligned}
$$

which can be expressed in terms of the Eisenstein series (1.1) to yield

$$
\ln \mathcal{Z}_{d, 1}(\tau, \bar{\tau})=\frac{\pi^{\frac{d-1}{2}} \Gamma\left(\frac{1-d}{2}\right)}{2} \frac{V_{d-1}}{L_{d}^{d-1}} \frac{f_{\frac{1-d}{2}}(\tau, \bar{\tau})}{\tau_{2}^{\frac{d-1}{2}}} .
$$

This agrees with (3.10) after using the reflection formula for Eisenstein series (3.8) at $z=\frac{d+1}{2}$.

Another way to obtain the heat kernel for a particle on a torus is by a standard path integral computation, in which case one finds instead

$$
\left\langle x^{\prime}\left|e^{-t \hat{\mathrm{H}}_{\beta, \alpha}}\right| x\right\rangle=\frac{1}{4 \pi t} \sum_{\left(m^{d}, m^{d+1}\right) \in \mathbb{Z}^{2}} e^{-\frac{1}{4 t} g},
$$

where

$$
\begin{aligned}
g= & \left(\alpha^{2}+\beta^{2}\right)\left(\frac{x_{d+1}^{\prime}-x_{d+1}}{\beta}+m^{d+1}\right)^{2}+2 \alpha L_{d}\left(\frac{x_{d+1}^{\prime}-x_{d+1}}{\beta}+m^{d+1}\right)\left(\frac{x_{d}^{\prime}-x_{d}}{L_{d}}+m^{d}\right) \\
& +L_{d}^{2}\left(\frac{x_{d}^{\prime}-x_{d}}{L_{d}}+m^{d}\right)^{2} .
\end{aligned}
$$


These two expressions for the heat kernel of a particle on a torus, (3.76) and (3.81) are related by the Poisson summation formula. When combining now the expression in (3.81) with the heat kernel in the $d-1$ large dimensions, one gets, after dropping the $m^{d}=0=m^{d+1}$ term,

$$
\ln \mathrm{Z}_{d, 1}(\beta, \alpha)=\frac{V_{d-1} L_{d} \beta}{2^{d+2} \pi^{\frac{d+1}{2}}} \sum_{\left(m^{d}, m^{d+1}\right) \in \mathbb{Z}^{2}}^{\prime} \int_{0}^{\infty} \mathrm{d} t t^{-\frac{d+3}{2}} e^{-\frac{1}{4 t} L_{d}^{2}\left|m^{d+1} \tau+m^{d}\right|^{2}} .
$$

In this case, the change of integration variable $t \rightarrow \frac{1}{t}$ gives

$$
\ln \mathrm{Z}_{d, 1}(\beta, \alpha)=\frac{V_{d-1} L_{d} \beta}{2^{d+2} \pi^{\frac{d+1}{2}}} \sum_{\left(m^{d}, m^{d+1}\right) \in \mathbb{Z}^{2}}^{\prime} \int_{0}^{\infty} \mathrm{d} t t^{\frac{d+1}{2}-1} e^{-\frac{t}{4} L_{d}^{2}\left|m^{d+1} \tau+m^{d}\right|^{2}} .
$$

Using now (2.65), the result can be shown directly to agree with (3.10) without using a reflection formula. Alternatively, the equivalence of both expressions (3.78) and (3.83) can be used to prove the reflection formula (3.8).

\section{Remarks}

(i) For a particle on a torus as in (3.75), one notes that under the canonical transformation

$$
\left\{\begin{array}{l}
p_{d+1}^{\prime}=p_{d+1}-\frac{\alpha}{\beta} p_{d}, \quad\left\{\begin{array}{l}
x_{d+1}^{\prime}=x_{d+1} \\
p_{d}^{\prime}=p_{d}
\end{array} x_{d}^{\prime}=x_{d}+\frac{\alpha}{\beta} x_{d+1}\right.
\end{array}\right.
$$

the Hamiltonian is diagonalized, $\hat{\mathrm{H}}_{\beta, \alpha}=\left(\hat{p}_{d+1}^{\prime}\right)^{2}+\left(\hat{p}_{d}^{\prime}\right)^{2}$, while the periodicities

$$
x_{d+1} \sim x_{d+1}+m^{d+1} \beta, \quad x_{d} \sim x_{d}+m^{d} L_{d},
$$

become

$$
x_{d+1}^{\prime} \sim x_{d+1}^{\prime}+m^{d+1} \beta, \quad x_{d}^{\prime} \sim x_{d}^{\prime}+m^{d} L_{d}+m^{d+1} \alpha .
$$

When $\alpha=0$, the role played by the canonical pairs $\left(x_{d+1}^{\prime}, p_{d+1}^{\prime}\right)$ and $\left(x_{d}^{\prime}, p_{d}^{\prime}\right)$ in the computation of $\operatorname{Tr} e^{-t \hat{\mathrm{H}}_{\beta, 0}}$ is symmetric if one exchanges at the same time the periodicities.

(ii) The Riemann theta function is defined by

$$
\vartheta_{n}(z \mid g)=\sum_{m^{\alpha} \in \mathbb{Z}^{n}} e^{\pi i m^{\alpha} g_{\alpha \beta} m^{\beta}+2 \pi i m^{\alpha} z_{\alpha}}, \quad \alpha, \beta=1, \ldots, n,
$$

for $g=g_{1}+i g_{2} \in \mathbb{H}_{n}=\left\{g \in M_{n}(\mathbb{C}) \mid g=g^{T}, g_{2}>0\right\}$ and $z \in \mathbb{C}^{n}$. Since $\left.\vartheta_{n=1}(z \mid g)\right|_{g=\tau}=\vartheta_{3}(z \mid \tau)$, the Riemann theta function is the higher dimensional generalization of the Jacobi theta function. One can also define

$$
\theta_{n}(g) \equiv \vartheta_{n}(0 \mid i g)=\sum_{m^{\alpha} \in \mathbb{Z}^{n}} e^{-\pi m^{\alpha} g_{\alpha \beta} m^{\beta}}
$$

Consider now the matrix

$$
\hat{g}_{\alpha \beta}=\frac{1}{\tau_{2}}\left(\begin{array}{cc}
1 & \tau_{1} \\
\tau_{1} & |\tau|^{2}
\end{array}\right)
$$


with unit determinant. After the change of variables $\frac{\tau_{2} L_{d}^{2}}{4 \pi t}=t^{\prime}$, the partition function in (3.83) can be written as

$$
\ln \mathcal{Z}_{d, 1}(\tau, \bar{\tau})=\frac{V_{d-1}}{2 L_{d}^{d-1} \tau_{2}^{\frac{d-1}{2}}} \int_{0}^{\infty} \mathrm{d} t t^{\frac{d+1}{2}-1}\left(\theta_{2}(t \hat{g})-1\right)
$$

Note that this suitably generalizes (2.70) to the case where the Euclidean spacetime manifold is $\mathbb{R}^{d-1} \times \mathbb{T}^{2}$. This integral expression makes modular covariance of the partition function transparent in the sense that the full behavior is governed by the prefactor alone since $\theta_{2}(\hat{g})$ is modular invariant. Indeed, since $m^{\alpha} \hat{g}_{\alpha \beta} m^{\beta}=$ $\frac{1}{\tau_{2}}\left|m^{d+1} \tau+m^{d}\right|^{2}$, the transformation $\tau^{\prime}=\tau+1$, can be absorbed by shifting the sum, $m^{d+1} \rightarrow m^{d+1}-1$, the transformation $\tau^{\prime}=-\frac{1}{\tau}$ results in the replacement of $\hat{g}$ by $\hat{g}^{-1}=\epsilon^{T} \hat{g}^{T} \epsilon$, with $\epsilon$ completely antisymmetric, and $\epsilon^{12}=1$, which can again be absorbed by re-arranging the sums.

Alternatively, the equivalence of the functional and world-line approaches to computing the partition function, i.e., the equality of the right hand sides of (3.13) and (3.91), shows that, at $z=d+1$, the completed real analytic Eisenstein series is directly related to the Mellin transform of the Riemann theta function minus one,

$$
\xi(z ; \tau, \bar{\tau})=\frac{1}{2} \int_{0}^{\infty} \mathrm{d} t t^{\frac{z}{2}-1}\left(\theta_{2}(t \hat{g})-1\right), \quad \mathfrak{R e}(z)>1
$$

\subsection{One spatial dimension}

In this section, we review for completeness the well-known result [42, 43] (see e.g. [44-46] for reviews) for a torus in Euclidean spacetime, that is to say we derive the partition function of a massless scalar field in a one spatial dimension, $d=1, L_{1}=L$, with periodic boundary conditions. As we have seen in section 3.1, a naive application of the functional approach leads one to the divergent expression

$$
\ln \mathcal{Z}_{1}(\tau, \bar{\tau})=\frac{1}{2 \pi} f_{1}(\tau, \bar{\tau})
$$

\subsubsection{Canonical approach}

In the analysis of section 2.1, the full Hamiltonian, including the $n=0$ mode, which is a free particle, is now given by

$$
H=\frac{1}{L} \frac{p^{2}}{2}+\frac{1}{2} \sum_{n \in \mathbb{Z}}^{\prime} \omega_{n}\left(a_{n}^{*} a_{n}+a_{n} a_{n}^{*}\right), \quad \omega_{n}=\frac{2 \pi|n|}{L} .
$$

After the conventional redefinition $p=\sqrt{4 \pi} a_{0}$, the quantum Hamiltonian including the zero point energy $E_{0}^{1}$ in $(2.77)$ can be written as

$$
\hat{H}=-\frac{\pi}{6 L}+\frac{2 \pi}{L}\left(\hat{a}_{0}^{2}+\sum_{n \in \mathbb{Z}}^{\prime}|n| \hat{a}_{n}^{\dagger} \hat{a}_{n}\right) .
$$


Keeping the finite part of (2.16), we get

$$
\begin{aligned}
\mathrm{Z}_{1}(\beta, \alpha) & =\sqrt{\frac{L}{2 \pi \beta}} e^{\frac{\pi \beta}{6 L}} \prod_{n \in \mathbb{Z}}^{\prime} \sum_{N_{n} \in \mathbb{N}} e^{\frac{2 \pi}{L}(-\beta|n|+i \alpha n) N_{n}} \\
& =\sqrt{\frac{L}{2 \pi \beta}} e^{\frac{\pi \beta}{6 L}} \prod_{n \in \mathbb{Z}}^{\prime} \frac{1}{1-e^{\frac{2 \pi}{L}(-\beta|n|+i \alpha n)}} .
\end{aligned}
$$

Defining

$$
q=e^{2 \pi i \tau}, \quad \bar{q}=e^{-2 \pi i \bar{\tau}},
$$

where $\tau$ and $\bar{\tau}$ are given in (3.4), we have

$$
\ln \mathcal{Z}_{1}(\tau, \bar{\tau})=-\frac{1}{2} \ln (2 \pi)+\frac{\pi \tau_{2}}{6}-\frac{1}{2} \ln \left(\tau_{2}\right)-\sum_{n \in \mathbb{N}^{*}} \ln \left[\left(1-q^{n}\right)\left(1-\bar{q}^{n}\right)\right] .
$$

In terms of Dedekind's eta function,

$$
\eta(q)=q^{\frac{1}{24}} \prod_{n \in \mathbb{N}^{*}}\left(1-q^{n}\right),
$$

and up to irrelevant numerical factors, the modular invariant partition function can then be written compactly as

$$
\mathcal{Z}_{1}(\tau, \bar{\tau})=\frac{1}{\sqrt{\tau_{2}}|\eta(q(\tau))|^{2}} .
$$

Note that in this case, since $\ln \mathcal{Z}_{1}(\tau, \bar{\tau})$ is the sum of a holomorphic and anti-holomorphic part, up to the contribution from the free particle,

$$
\Delta_{\mathbb{H}} \ln \mathcal{Z}_{1}(\tau, \bar{\tau})=\frac{1}{2} .
$$

This is not the formal continuation of (3.35) to $d=1$ because of the contribution of the free particle, which gives rise to the factor $1 / 2$ on the right hand side. Hence, in this case,

$$
\left\langle(\Delta \hat{H})^{2}-(\Delta \hat{P})^{2}\right\rangle=\frac{1}{2 \beta^{2}}, \quad\left\langle\hat{L}_{0} \hat{\bar{L}}_{0}\right\rangle-\left\langle\hat{L}_{0}\right\rangle\left\langle\hat{\bar{L}}_{0}\right\rangle=-\frac{1}{8 \pi^{2}(\tau-\bar{\tau})^{2}} .
$$

\subsubsection{Dimensional continuation}

A different way to give a meaning the non-convergent expression of the partition function (3.93) obtained in the functional or heat kernel approach is to study the limit $s \rightarrow 1$ of the real analytic Eisenstein series $f_{s}(\tau, \bar{\tau})$ by starting from its Fourier expansion [4] given in equation (3.64),

$$
\begin{aligned}
f_{s}(\tau, \bar{\tau})= & 2 \zeta(2 s) \tau_{2}^{s}+2 \sqrt{\pi} \frac{\Gamma\left(s-\frac{1}{2}\right) \zeta(2 s-1)}{\Gamma(s)} \tau_{2}^{1-s} \\
& +2 \frac{\pi^{s}}{\Gamma(s)} \tau_{2}^{\frac{1}{2}} \sum_{n \in \mathbb{Z}}^{\prime} \sum_{m \in \mathbb{Z}}^{\prime}\left|\frac{n}{m}\right|^{s-\frac{1}{2}} K_{s-\frac{1}{2}}\left(2 \pi|n m| \tau_{2}\right) e^{2 \pi i n|m| \tau_{1}}
\end{aligned}
$$


Only the second term diverges in the limit $s \rightarrow 1$, while the first term on the right hand side becomes

$$
\frac{\pi^{2}}{3} \tau_{2}=-\frac{\pi}{12} \ln |q|^{2}
$$

Using $K_{\frac{1}{2}}(z)=e^{-z} \sqrt{\frac{\pi}{2 z}}$, the term on the last line becomes

$$
\begin{aligned}
\pi \sum_{n \in \mathbb{Z}}^{\prime} \sum_{m \in \mathbb{Z}}^{\prime} \frac{1}{|m|} e^{-2 \pi|n m| \tau_{2}+2 \pi i n|m| \tau_{1}} & =2 \pi \sum_{m \in \mathbb{N}^{*}} \sum_{n \in \mathbb{N}^{*}} \frac{1}{m}\left(e^{2 \pi i n m \tau}+e^{-2 \pi i n m \bar{\tau}}\right) \\
& =-2 \pi \sum_{n \in \mathbb{N}^{*}}\left[\ln \left(1-e^{2 \pi i n \tau}\right)+\ln \left(1-e^{-2 \pi i n \bar{\tau}}\right)\right] \\
& =-2 \pi \ln \prod_{n \in \mathbb{N}^{*}}\left(1-q^{n}\right) \prod_{n \in \mathbb{N}^{*}}\left(1-\bar{q}^{n}\right)
\end{aligned}
$$

These terms combine into

$$
-2 \pi \ln \prod_{n \in \mathbb{N}^{*}} q^{\frac{1}{24}}\left(1-q^{n}\right) \prod_{n \in \mathbb{N}^{*}} \frac{1}{24}\left(1-\bar{q}^{n}\right)=-2 \pi \ln |\eta(q)|^{2} .
$$

For the divergent term, we consider the expansion around $s=1$ using

$$
\zeta(s)=\frac{1}{s-1}+\gamma+O(s-1)
$$

so that

$$
\begin{aligned}
2 \sqrt{\pi} \frac{\Gamma\left(s-\frac{1}{2}\right) \zeta(2 s-1)}{\Gamma(s)} \tau_{2}^{1-s} & =\frac{\pi}{s-1}+\pi\left(3 \gamma+\frac{\Gamma^{\prime}\left(\frac{1}{2}\right)}{\Gamma\left(\frac{1}{2}\right)}-\ln \tau_{2}\right)+O(s-1) \\
& =\frac{\pi}{s-1}+\pi\left(2 \gamma-2 \ln 2-\ln \tau_{2}\right)+O(s-1) .
\end{aligned}
$$

We thus get

$$
\lim _{s \rightarrow 1}\left(f_{s}(\tau, \bar{\tau})-\frac{\pi}{s-1}\right)=2 \pi\left(\gamma-\ln 2-\ln \sqrt{\tau_{2}}|\eta(q)|^{2}\right) .
$$

Therefore, once we remove the divergence, the real analytic Eisenstein series for $s=1$ contains the result for the partition function of the $d=1$ case. Note that the divergence in the second term of the expansion in (3.103), comes from the $n_{1}=0$ term in the mode expansion of the field (at fixed time), as shown in the canonical approach in the previous section. In $d>1$ this mode is a scalar field in $d-1$ spatial dimensions. For $d=1$, it is a free particle, which gives the factor $\sqrt{\tau_{2}}$ needed for modular invariance. Associated with the divergence, the finite, $\tau_{2}$ independent term, is undetermined and needs to be fixed by a normalization condition.

How this computation appears from the viewpoint of the integral representation connected to the world-line approach is discussed in [43]. 


\section{$4 \quad$ Higher dimensional tori}

\subsection{Generalities}

We now consider the more general case of the Euclidean spacetime manifold $\mathbb{R}^{p} \times \mathbb{T}^{q+1}[6,12]$, with $p+q=d$. Coordinates on $\mathbb{R}^{p}$ are denoted by $x^{I}, I=1, \ldots, p$. The skewed torus $\mathbb{T}^{q+1}$ is defined to be the quotient of $\mathbb{R}^{q+1}$ with coordinates $x^{a}=\left(x^{i}, x^{d+1}\right), i=p+1, \ldots d$, by the lattice $\Lambda^{d+1}$ generated by a set of $q+1$ constant frame vectors $e^{a}{ }_{\alpha} \in \mathbb{R}^{d+1}$,

$$
\mathbb{T}^{q+1}=\mathbb{R}^{q+1} / \Lambda^{q+1}, \quad \Lambda^{q+1}=\left\{m^{\alpha} e^{a}{ }_{\alpha} \mid m^{\alpha} \in \mathbb{Z}^{q+1}\right\},
$$

with $\alpha=1, \ldots, q+1$.

Functions on this manifold satisfy the periodicity conditions

$$
\phi\left(x^{I}, x^{a}\right)=\phi\left(x^{I}, x^{a}+e^{a}{ }_{\alpha}\right), \quad \forall \alpha
$$

In $x^{a}$ coordinates, the information on the geometry of the torus is thus encoded in the skewed periodicities determined by the frame vectors $e^{a}{ }_{\alpha}$ whereas the Lagrangian Euclidean action is

$$
S_{L}^{E}[\phi]=\frac{1}{2} \int_{V_{d+1}} \mathrm{~d}^{d+1} x\left[\partial_{I} \phi \partial^{I} \phi+\delta^{a b} \partial_{a} \phi \partial_{b} \phi\right] .
$$

Associated to the $q+1$ linearly independent frame vectors, there is a co-frame $e_{a}{ }^{\alpha}$ such that

$$
e_{\alpha}^{a} e_{b}^{\alpha}=\delta_{b}^{a}, \quad e_{\alpha}^{a} e_{a}^{\beta}=\delta_{\alpha}^{\beta} .
$$

This allows one to define coordinates $y^{\alpha}=e_{a}{ }^{\alpha} x^{a}$ in which periodicities of the field become simply unity in all these directions separately,

$$
\phi\left(x^{I}, y^{\alpha}\right)=\phi\left(x^{I}, y^{\alpha}+\delta_{\beta}^{\alpha}\right), \quad \forall \beta .
$$

In turn, the Cartesian metric $\delta_{a b}$ becomes

$$
g_{\alpha \beta}=e_{\alpha}^{a} \delta_{a b} e^{b} .
$$

When working in $y^{\alpha}$ coordinates, the information on the non-trivial geometry of the torus gets encoded in the Lagrangian action through the coupling of the scalar field to the metric $g_{\alpha \beta}$,

$$
S_{L}^{E}[\phi ; g]=\frac{1}{2} \int_{V_{d+1}} \mathrm{~d}^{p} x \mathrm{~d}^{q+1} y \sqrt{g}\left[g^{\alpha \beta} \partial_{\alpha} \phi \partial_{\beta} \phi+\partial_{I} \phi \partial^{I} \phi\right] .
$$

Note that since the metric is constant and flat, there is no need here to consider the conformally coupled scalar field with its improved energy-momentum tensor, as usually done in the case of a general curved background (see e.g. [12, 35, 47]). The volume of the torus is $\mathrm{V}_{q+1}=\operatorname{det} e^{a}{ }_{\alpha}=\sqrt{g}$. For later use, we define the re-scaled frame, co-frame, metric and inverse metric,

$$
\begin{array}{ll}
\hat{e}^{a}{ }_{\alpha}=\left(\mathrm{V}_{q+1}\right)^{-\frac{1}{q+1}} e^{a}{ }_{\alpha}, & \hat{e}_{a}{ }^{\alpha}=\left(\mathrm{V}_{q+1}\right)^{\frac{1}{q+1}} e_{a}{ }^{\alpha}, \\
\hat{g}_{\alpha \beta}=\left(\mathrm{V}_{q+1}\right)^{-\frac{2}{q+1}} g_{\alpha \beta}, & \hat{g}^{\alpha \beta}=\left(\mathrm{V}_{q+1}\right)^{\frac{2}{q+1}} g^{\alpha \beta},
\end{array}
$$


all of which have unit determinant.

Even though we will not need this construction here, we note that for the dual lattice, the periodicities in $x_{a}$ coordinates are defined instead by the vectors $e_{a}{ }^{\alpha}$. The coordinates in which these periodicities all become unity separately in all directions are $y_{\alpha}=x_{a} e^{a}$. The metric associated with the dual torus is $g^{\alpha \beta}$, the inverse of the metric associated with the original torus.

\subsection{Functional approach}

The aim is now to compute the partition function of the massless scalar field in this flat background,

$$
Z_{d, q}(g)=\int \prod_{x^{I}, y^{\alpha}} d \phi\left(x^{I}, y^{\alpha}\right) e^{-S_{L}^{E}[\phi ; g]}
$$

As before, we treat $\mathbb{R}^{p}$ by first considering $\mathbb{S}_{L_{1}}^{1} \times \cdots \times \mathbb{S}_{L_{p}}^{1}$ with coordinates $x^{I}, I=1, \ldots p$, so that the fields satisfy the periodicity conditions $\phi\left(x^{I}+L_{I}, y^{\alpha}\right)=\phi\left(x^{I}, y^{\alpha}\right)$. The passage to $\mathbb{R}^{p}$ is done by replacing sums by integrals at the expense of a factor $(2 \pi)^{-p} V_{p}, V_{p}=\prod_{I=1}^{p} L_{I}$. On $\mathbb{S}_{L_{1}}^{1} \times \cdots \times \mathbb{S}_{L_{p}}^{1} \times \mathbb{T}^{q+1}$, an orthonormal basis of functions is

$$
e_{n_{I}, n_{\alpha}}(x, y)=\frac{1}{\sqrt{V_{d+1}}} e^{k_{I} x^{I}+2 \pi i n_{\alpha} y^{\alpha}}
$$

with $k_{I}=\frac{2 \pi n_{I}}{L_{I}}, n_{I} \in \mathbb{Z}^{p}, n_{\alpha} \in \mathbb{Z}^{q+1}$, and $V_{d+1}=V_{d-q} \mathrm{~V}_{q+1}$. The orthonormality condition is

$$
\begin{aligned}
\left(e_{n_{I}, n_{\alpha}}, e_{n_{I}^{\prime}, n_{\alpha}^{\prime}}\right) & =\int_{V_{d+1}} \mathrm{~d}^{p} x \mathrm{~d}^{q+1} y \sqrt{g} e_{n_{I}, n_{\alpha}}^{*}(x, y) e_{n_{I}^{\prime}, n_{\alpha}^{\prime}}(x, y) \\
& =\frac{1}{V_{d+1}} \int_{V_{d+1}} \mathrm{~d}^{p} x \mathrm{~d}^{q+1} y \sqrt{g} e^{i\left(k_{I}^{\prime}-k_{I}\right) x^{I}} e^{2 \pi i\left(n_{\alpha}^{\prime}-n_{\alpha}\right) y^{\alpha}}=\prod_{I, \alpha} \delta_{n_{I}, n_{I}^{\prime}} \delta_{n_{\alpha}, n_{\alpha}^{\prime}} .
\end{aligned}
$$

These basis functions are eigenfunctions of the Laplacian,

$$
\begin{aligned}
\Delta & =\partial_{I} \partial^{I}+g^{\alpha \beta} \partial_{\alpha} \partial_{\beta}, \\
\Delta e_{n_{I}, n_{\alpha}} & =-\lambda_{n_{I}, n_{\alpha}}^{2} e_{n_{I}, n_{\alpha}}, \quad \lambda_{n_{I}, n_{\alpha}}^{2}=k_{I} k^{I}+(2 \pi)^{2} n_{\alpha} g^{\alpha \beta} n_{\beta} .
\end{aligned}
$$

When taking into account that the $p$ first dimensions become large, the associated zeta function is

$$
\begin{aligned}
& \zeta_{-\Delta}(s)=\frac{V_{p}}{(2 \pi)^{p}} \int \mathrm{d}^{p} k \sum_{n_{\alpha} \in \mathbb{Z}^{q+1}}^{\prime}\left[(2 \pi)^{2} n_{\alpha} g^{\alpha \beta} n_{\beta}+k_{I} k^{I}\right]^{-s}=\frac{V_{p}}{(2 \pi)^{p}} \frac{2 \pi^{\frac{p}{2}}}{\Gamma\left(\frac{p}{2}\right)} \\
& \sum_{n_{\alpha} \in \mathbb{Z}^{q+1}}^{\prime} \int_{0}^{\infty} \mathrm{d} k k^{p-1}\left[(2 \pi)^{2} n_{\alpha} g^{\alpha \beta} n_{\beta}+k^{2}\right]^{-s}=\frac{V_{p} \pi^{\frac{p}{2}-2 s} \Gamma\left(s-\frac{p}{2}\right)}{2^{2 s} \Gamma(s)} \sum_{n_{\alpha} \in \mathbb{Z}^{q+1}}^{\prime}\left[n_{\alpha} g^{\alpha \beta} n_{\beta}\right]^{\frac{p}{2}-s} \\
& =\frac{V_{p} \pi^{\frac{p}{2}-2 s} \Gamma\left(s-\frac{p}{2}\right)}{2^{2 s} \Gamma(s)\left(\mathrm{V}_{q+1}\right)^{\frac{p-2 s}{q+1}}} \sum_{n_{\alpha} \in \mathbb{Z}^{q+1}}^{\prime}\left[n_{\alpha} \hat{g}^{\alpha \beta} n_{\beta}\right]^{\frac{p}{2}-s}=\frac{V_{p} \pi^{\frac{p}{2}-2 s} \Gamma\left(s-\frac{p}{2}\right)}{2^{2 s} \Gamma(s)\left(\mathrm{V}_{q+1}\right)^{\frac{p-2 s}{q+1}}} f_{s-\frac{p}{2}}\left(q+1 ; \hat{g}^{-1}\right) .
\end{aligned}
$$


In the last line, we have identified the sum in terms of the $\operatorname{SL}(n, \mathbb{Z})$ Eisenstein series in equation (2.6) of [1] and denoted for notational coherence here by $f_{s}\left(n ; \hat{g}^{-1}\right)$,

$$
f_{s}\left(n ; \hat{g}^{-1}\right)=\sum_{n_{\alpha} \in \mathbb{Z}^{n}}^{\prime}\left[n_{\alpha} \hat{g}^{\alpha \beta} n_{\beta}\right]^{-s}, \quad \mathfrak{R e}(s)>\frac{n}{2} .
$$

As shown in [1], this series satisfies the reflection formula

$$
\pi^{-s} \Gamma(s) f_{s}\left(n ; \hat{g}^{-1}\right)=\pi^{s-\frac{n}{2}} \Gamma\left(\frac{n}{2}-s\right) f_{\frac{n}{2}-s}(n ; \hat{g}),
$$

where

$$
f_{s}(n ; \hat{g})=\sum_{m^{\alpha} \in \mathbb{Z}^{n}}^{\prime}\left[m^{\alpha} \hat{g}_{\alpha \beta} m^{\beta}\right]^{-s}, \quad \mathfrak{R e}(s)>\frac{n}{2} .
$$

When applied to (4.14), we get

$$
\zeta_{-\Delta}(s)=\frac{\Gamma\left(\frac{p+q+1}{2}-s\right) V_{p}}{2^{2 s} \Gamma(s) \pi^{\frac{p+q+1}{2}}\left(\mathrm{~V}_{q+1}\right)^{\frac{p-2 s}{q+1}}} f_{\frac{p+q+1}{2}-s}(q+1 ; \hat{g}),
$$

and then, using (2.33) together with $p+q=d$,

$$
\ln Z_{d, q}(g)=\frac{\Gamma\left(\frac{d+1}{2}\right) V_{d-q}}{2 \pi^{\frac{d+1}{2}}\left(\mathrm{~V}_{q+1}\right)^{\frac{d-q}{q+1}}} f_{\frac{d+1}{2}}(q+1 ; \hat{g}) .
$$

In terms of the completed $\operatorname{SL}(n, \mathbb{Z})$ Eisenstein series

$$
\xi(z, n ; \hat{g})=\frac{\Gamma\left(\frac{z}{2}\right) f_{\frac{z}{2}}(n ; \hat{g})}{2 \pi^{\frac{z}{2}}},
$$

which satisfies the reflection formula

$$
\xi\left(z, n ; \hat{g}^{-1}\right)=\xi(n-z, n ; \hat{g}),
$$

the partition function becomes

$$
\ln Z_{d, q}(g)=\frac{V_{d-q}}{\left(\mathrm{~V}_{q+1}\right)^{\frac{d-q}{q+1}}} \xi(d+1, q+1 ; \hat{g}) .
$$

\section{Remarks}

(i) The above derivation is only valid for $p>1, q<d$. Indeed, in the case where there are no large spatial dimensions, $p=0, q=d$, the expression

$$
\ln Z_{d, d}(g)=\xi(d+1, d+1 ; \hat{g})
$$

diverges. As emphasized in section 2.2.1, this is due to the presence of the zero mode, whose contribution cannot be neglected when there are no large dimensions. A renormalized expression, relying on the canonical approach of section 4.3, is provided in section 4.5. 
(ii) The result (4.22) can be expressed more simply in terms of the completed GL( $n, \mathbb{Z})$ Eisenstein series $\xi(z, n ; g)$, which has the same definition as the $\operatorname{SL}(n, \mathbb{Z})$ one in equation (4.20) but with $\hat{g}_{\alpha \beta}$ replaced by $g_{\alpha \beta}$, and satisfies the inversion formula

$$
\begin{aligned}
\xi\left(z, n ; g^{-1}\right) & =\mathrm{V}_{n} \xi(n-z, n ; g), \\
\ln Z_{d, q}(g) & =V_{d-q} \mathrm{~V}_{q+1} \xi(d+1, q+1 ; g) .
\end{aligned}
$$

(iii) The $\mathrm{SL}(n, \mathbb{Z})$ Eisenstein series is invariant under $\mathrm{SL}(n, \mathbb{Z})$ transformations of the lattice vectors defining $\mathbb{T}^{n}$ : if $S_{\alpha}{ }^{\beta} \in \mathrm{SL}(n, \mathbb{Z})$ and

$$
e^{\prime a}{ }_{\alpha}=S_{\alpha}^{\beta} e^{a}{ }_{\beta}, \quad g_{\alpha \beta}^{\prime}=S_{\alpha}^{\gamma} g_{\gamma \delta} S_{\beta}^{\delta},
$$

then

$$
\begin{aligned}
f_{s}\left(n ; \hat{g}^{\prime}\right) & =\sum_{m^{\alpha} \in \mathbb{Z}^{n}}^{\prime}\left[m^{\alpha} \hat{g}_{\alpha \beta}^{\prime} m^{\beta}\right]^{-s}=\sum_{m^{\alpha} \in \mathbb{Z}^{n}}^{\prime}\left[m^{\alpha} S_{\alpha}{ }^{\gamma} \hat{g}_{\gamma \delta} S_{\beta}{ }^{\delta} m^{\beta}\right]^{-s} \\
& =\sum_{m^{\prime \alpha} \in \mathbb{Z}^{n}}^{\prime}\left[m^{\prime \gamma} \hat{g}_{\gamma \delta} m^{\prime \delta}\right]^{-s}=f_{s}(n ; \hat{g}),
\end{aligned}
$$

where $m^{\prime \gamma}=m^{\alpha} S_{\alpha} \gamma$. A similar proof holds for $f_{s}\left(n ; \hat{g}^{\prime-1}\right)=f_{s}\left(n ; \hat{g}^{-1}\right)$. Since both the volume of $\mathbb{T}^{q+1}$ and $\xi(d+1, q+1 ; \hat{g})$, are separately $\operatorname{SL}(q+1, \mathbb{Z})$ invariant, so is the full partition function in (4.22).

(iv) In order to make the connection with the discussion in section 2.3, one should introduce, instead of $y^{\alpha}$, coordinates $y^{\prime \alpha}=L_{(\alpha)} y^{\alpha}$ with $L_{d+1}=\beta$, so that the periodicities in (4.5) get replaced by

$$
\phi\left(y^{\prime \alpha}\right)=\phi\left(y^{\prime \alpha}+L_{\alpha}\right), \quad \forall \alpha .
$$

In $y^{\prime \alpha}$ coordinates, the Euclidean action in (2.29) corresponds to the particular choice of metric given by $g^{\prime i j}=\delta^{i j}$ and $g^{\prime d+1, i}=-\mu^{i}$, where $i=1, \ldots, d$.

(v) For $p=d$ and $q=0$,

$$
f_{s}(1 ; \hat{g})=2 \zeta(2 s), \quad \xi(z, 1 ; \hat{g})=\xi(z), \quad \hat{g}=1,
$$

and (4.22) reduces to the scalar black body result (2.24).

For $p=d-1$ and $q=1,(4.22)$ reduces to the partition function on $\mathbb{R}^{d-1} \times \mathbb{T}^{2}$ in (3.10) covariant under the $\mathrm{SL}(2, \mathbb{Z}) / \mathbb{Z}^{2}$ transformations of the modular parameter in (1.2). Indeed, when using the parametrization

$$
\begin{aligned}
& \hat{e}^{a}{ }_{\alpha}=\frac{1}{\sqrt{L_{d} \beta}}\left(\begin{array}{cc}
L_{d} & \alpha \\
0 & \beta
\end{array}\right)=\frac{1}{\sqrt{\tau_{2}}}\left(\begin{array}{cc}
1 & \tau_{1} \\
0 & \tau_{2}
\end{array}\right), \\
& \hat{e}_{a}{ }^{\alpha}=\frac{1}{\sqrt{L_{d} \beta}}\left(\begin{array}{cc}
\beta & 0 \\
-\alpha & L_{d}
\end{array}\right)=\frac{1}{\sqrt{\tau_{2}}}\left(\begin{array}{cc}
\tau_{2} & 0 \\
-\tau_{1} & 1
\end{array}\right), \\
& \hat{g}_{\alpha \beta}=\frac{1}{L_{d} \beta}\left(\begin{array}{cc}
L_{d}^{2} & L_{d} \alpha \\
L_{d} \alpha & \alpha^{2}+\beta^{2}
\end{array}\right)=\frac{1}{\tau_{2}}\left(\begin{array}{cc}
1 & \tau_{1} \\
\tau_{1} & |\tau|^{2}
\end{array}\right), \\
& \hat{g}^{\alpha \beta}=\frac{1}{L_{d} \beta}\left(\begin{array}{cc}
\alpha^{2}+\beta^{2} & -L_{d} \alpha \\
-L_{d} \alpha & L_{d}^{2}
\end{array}\right)=\frac{1}{\tau_{2}}\left(\begin{array}{cc}
|\tau|^{2} & -\tau_{1} \\
-\tau_{1} & 1
\end{array}\right),
\end{aligned}
$$


so that

$$
f_{s}(2 ; \hat{g})=\sum_{\left(m^{1}, m^{2}\right) \in \mathbb{Z}^{2}}^{\prime} \frac{\tau_{2}^{s}}{\left|m^{2} \tau+m^{1}\right|^{2 s}}=f_{s}(\tau, \bar{\tau}),
$$

is the real analytic Eisenstein series of (1.1). In addition, since

$$
\tau=\frac{e_{d+1}^{d}+i e^{d+1} d+1}{e_{d}^{d}+i e^{d+1}},
$$

the modular transformation (1.2) originates from an $\mathrm{SL}(2, \mathbb{Z})$ transformation of the lattice vectors generating $\mathbb{T}^{2}$ of the form

$$
S_{\alpha}^{\beta}=\left(\begin{array}{ll}
d & c \\
b & a
\end{array}\right) .
$$

(vi) The reflection formula (4.16) reduces to the one satisfied by the real analytic Eisenstein series $f_{s}(\tau, \bar{\tau})$ in (3.8) for $n=2$. Indeed, in this case, since $\hat{g}^{-1}=\epsilon^{T} \hat{g}^{T} \epsilon$, with $\epsilon \in \mathrm{SL}(2, \mathbb{Z})$ completely antisymmetric, and $\epsilon^{12}=1$, it follows in particular that $f_{s}(2 ; \hat{g})=f_{s}\left(2 ; \hat{g}^{-1}\right)$. For $n=1$, formula (4.16) reduces to the reflection formula (2.42) for the Riemann zeta function.

(vii) As shown in appendix $\mathrm{A}$, the $\mathrm{SL}(n, \mathbb{Z})$ and $\mathrm{GL}(n, \mathbb{Z})$ Eisenstein series are eigenfunctions of suitable Laplacians. In order to understand the interpretation of these relations in terms of the partition function, we introduce suitable observables, an integrated energy momentum tensor,

$$
\mathcal{T}_{\alpha \beta}=\frac{2}{\sqrt{g}} \frac{\partial S_{L}^{E}[\phi ; g]}{\partial g^{\alpha \beta}}=\int \mathrm{d}^{p} x \mathrm{~d}^{q+1} y\left[\partial_{\alpha} \phi \partial_{\beta} \phi-\frac{1}{2} g_{\alpha \beta}\left(\partial_{\gamma} \phi \partial^{\gamma} \phi+\partial_{I} \phi \partial^{I} \phi\right)\right],
$$

as well as integrated bilinears in first order derivatives of the field in the small direction,

$$
\mathcal{D}_{\alpha \beta}=\int \mathrm{d}^{p} x \mathrm{~d}^{q+1} y \partial_{\alpha} \phi \partial_{\beta} \phi .
$$

When using $\sqrt{g}=t^{\frac{q+1}{2}}=\mathrm{V}_{q+1}$, these definitions imply that

$$
\begin{aligned}
& \frac{\partial S_{L}^{E}[\phi ; g]}{\partial \hat{g}_{\alpha \beta}}=-\frac{\sqrt{g}}{2} t J_{\gamma \delta}^{\alpha \beta} \mathcal{T}^{\gamma \delta}=-t^{\frac{q-1}{2}} \hat{J}^{\alpha \beta \gamma \delta} \mathcal{D}_{\gamma \delta}, \\
& t \frac{\partial S_{L}^{E}[\phi ; g]}{\partial t}=-\frac{1}{2} t^{\frac{q+1}{2}} g^{\alpha \beta} \mathcal{T}_{\alpha \beta},
\end{aligned}
$$

where the tensor $\hat{J}^{\alpha \beta \gamma \delta}$ is defined in appendix A, with $n=q+1$ here. It then follows from (A.18) and (4.22) that

$$
\begin{aligned}
& (q+1) t^{q-1} \hat{J}^{\alpha \beta \gamma \delta}\left\langle\Delta \hat{\mathcal{D}}_{\alpha \beta} \Delta \hat{\mathcal{D}}_{\gamma \delta}\right\rangle-q(q+3) t^{\frac{q-1}{2}} \hat{g}^{\alpha \beta}\left\langle\hat{\mathcal{D}}_{\alpha \beta}\right\rangle \\
& =q(d-q)(d+1) \ln Z_{d, q}(g),
\end{aligned}
$$

while

$$
g^{\alpha \beta}\left\langle\hat{\mathcal{T}}_{\alpha \beta}\right\rangle=(q-d) \frac{1}{\mathrm{~V}_{q+1}} \ln Z_{d, q}(g)
$$


(viii) It is of interest to see whether $\operatorname{SL}(q+1, \mathbb{Z})$ invariance of the partition function in (4.22) could have been anticipated from the path integral definition (4.9) before doing the actual computation. From the viewpoint of Ward identities, when concentrating on transformations of the coordinates $y^{\alpha}$ associated with the torus alone, a possible argument goes as follows. The action $S_{L}^{E}[\phi ; g]$ is invariant under diffeomorphisms, $y^{\prime \alpha}=y^{\prime \alpha}\left(y^{\beta}\right), S_{L}^{E}\left[\phi^{\prime}, g^{\prime}\right]=S_{L}^{E}[\phi, g]$, when $\phi$ transforms as a scalar and $g_{\alpha \beta}$ as a tensor field. A standard change of integration variables in the path integral then would imply that $Z\left[g^{\prime}\right]=Z[g]$ provided that the path integral measure is invariant. This formal argument does however not take the boundary conditions into account. Besides the action $S_{L}^{E}[\phi ; g]$, the theory is also defined by the boundary conditions (4.5). When neglecting the zero mode, the path integral measure is defined in terms of the Fourier coefficients for the periodic paths. It thus stays invariant if the paths stay periodic with the same periodicities, $\phi^{\prime}\left(y^{\prime \alpha}+\delta_{\beta}^{\alpha}\right)=\phi^{\prime}\left(y^{\prime \alpha}\right)$, or equivalently, $\phi\left(y^{\alpha}\left(y^{\prime \beta}+\delta_{\gamma}^{\beta}\right)\right)=\phi\left(y^{\alpha}\left(y^{\prime \beta}\right)\right)$. In turn, this requires $y^{\alpha}\left(y^{\prime \beta}+\delta_{\gamma}^{\beta}\right)=y^{\alpha}\left(y^{\prime \beta}\right)+m^{\alpha}$, with $m^{\alpha} \in \mathbb{Z}$, and then that $y^{\alpha}=\sum_{n_{\beta}^{\prime} \in \mathbb{Z}^{q+1}} Y_{n_{\beta}^{\prime}}^{\alpha} e^{2 \pi i n_{\beta}^{\prime} y^{\prime \beta}}+S_{\gamma}^{\alpha} y^{\prime \gamma}$ with $S_{\gamma}^{\alpha} \in \operatorname{SL}(q+1, \mathbb{Z})$. When restricting to $y^{\alpha}=Y_{0}^{\alpha}+S^{\alpha}{ }_{\gamma} y^{\prime \gamma}$ guarantees that the associated transformation of the metric remains constant.

\subsection{Canonical approach}

In order to set up the Hamiltonian analysis, we choose the ADM parametrization (see [48] for a review) of the metric adapted to Euclidean signature,

$$
g_{\alpha \beta}^{\mathrm{ADM}}=\left(\begin{array}{cc}
h_{\iota \kappa} & N_{\kappa} \\
N_{\iota} & N^{2}+N_{\lambda} N^{\lambda}
\end{array}\right), \quad g_{\mathrm{ADM}}^{\alpha \beta}=\left(\begin{array}{cc}
h^{\iota \kappa}+\frac{N^{\iota} N^{\kappa}}{N^{2}} & -\frac{N^{\kappa}}{N^{2}} \\
-\frac{N^{\iota}}{N^{2}} & \frac{1}{N^{2}}
\end{array}\right),
$$

where $\iota=d-q+1, \ldots, d$ and $N_{\iota}=h_{\iota \kappa} N^{\kappa}$, so that

$$
\begin{aligned}
S_{L}^{E}\left[\phi ; g^{\mathrm{ADM}}\right]=\frac{1}{2} \int_{V_{d+1}} \mathrm{~d}^{p} x \mathrm{~d}^{q+1} y & {\left[\frac{\sqrt{h}}{N}\left(\partial_{d+1} \phi-N^{\iota} \partial_{\iota} \phi\right)^{2}\right.} \\
& \left.+N \sqrt{h}\left(h^{\iota \kappa} \partial_{\iota} \phi \partial_{\kappa} \phi+\partial_{I} \phi \partial^{I} \phi\right)\right],
\end{aligned}
$$

with associated first order action

$$
S_{H}^{E}\left[\phi, \pi ; g^{\mathrm{ADM}}\right]=\int_{V_{d+1}} \mathrm{~d}^{p} x \mathrm{~d}^{q+1} y\left[-i \pi \partial_{d+1} \phi+N \mathcal{H}(h)+i N^{\iota} \mathcal{H}_{\iota}(h)\right],
$$

where

$$
\mathcal{H}(h)=\frac{1}{2 \sqrt{h}} \pi^{2}+\frac{1}{2} \sqrt{h}\left(h^{\iota \kappa} \partial_{\iota} \phi \partial_{\kappa} \phi+\partial_{I} \phi \partial^{I} \phi\right), \quad \mathcal{H}_{\iota}(h)=\pi \partial_{\iota} \phi .
$$

A moving frame associated to the ADM form of the metric is

$$
e_{\mathrm{ADM}}{ }^{a} \alpha=\left(\begin{array}{cc}
\theta^{i}{ }_{\iota} & \theta^{i}{ }_{\kappa} N^{\kappa} \\
0 \ldots 0 & N
\end{array}\right), \quad e^{\mathrm{ADM} \alpha}{ }_{a}^{\alpha}=\left(\begin{array}{cc} 
& 0 \\
\theta_{i}{ }^{\iota} & \vdots \\
& 0 \\
-\frac{N^{\iota}}{N} & \frac{1}{N}
\end{array}\right),
$$


where $\theta_{\iota}{ }_{\iota}$ is a moving frame for the spatial metric,

$$
h_{\iota \kappa}=\theta^{i}{ }_{\iota} \delta_{i j} \theta^{j}{ }_{\kappa} .
$$

In this parametrization, if we $\operatorname{define} \operatorname{det} \theta^{i}{ }_{\iota}=\sqrt{h}=\mathrm{V}_{q}$, we have $\operatorname{det} e_{\mathrm{ADM}}{ }^{a}{ }_{\alpha}=N \mathrm{~V}_{q}$ and $\operatorname{det} g_{\alpha \beta}^{\mathrm{ADM}}=N^{2} \mathrm{~V}_{q}^{2}$.

If an explicit parametrization of the spatial metric is needed as well, one may continue to parametrize both the spatial metric and the associated moving frame in ADM form with lapse and shift $N_{(q)}, N_{(q)}^{\iota_{d}-1}$, with $\iota_{d-1}=d-q+1, \ldots, d-1$ until one reaches $(q)=d-q+1$, in which case only the lapse $N_{(d-q+1)}$ remains. Note that this yields a parametrization of $e_{\mathrm{ADM}}^{a} \alpha$ of Iwasawa type,

$$
e_{\mathrm{ADM}}^{a}{ }_{\alpha}=(\mathcal{K} \mathcal{A N})^{a}{ }_{\alpha},
$$

where the $\mathrm{SO}(q+1, \mathbb{R})$ element $\mathcal{K}$ is the unit matrix, $\mathcal{K}=\mathbf{1}, \mathcal{A}=\operatorname{diag}\left(N_{(d-q+1)}, \ldots N_{(d)}, N=\right.$ $N_{(d+1)}$ ), while $\mathcal{N}$ is upper diagonal with 1's on the diagonal. In this parametrization, (and also with a more general Iwasawa parametrization including a non-trivial $\mathrm{SO}(q+1, \mathbb{R})$ element that does not contribute to the ADM metric by construction), the volume of the torus $\mathbb{T}^{q+1}$ is simply the product of the lapses, $\mathrm{V}_{q+1}=\prod_{\alpha=d-q+1}^{d+1} N_{(\alpha)}$.

Let us define

$$
H(h)=\int_{V_{d}} \mathrm{~d}^{p} x \mathrm{~d}^{d} y \mathcal{H}(h) .
$$

When using that $\pi$ is a tensor density under spatial coordinate transformations,

$$
\pi\left(x^{I}, y^{\iota}, y^{d+1}\right)=\sqrt{h} \pi\left(x^{I}, x^{i}, y^{d+1}\right),
$$

it follows that $H(h)$ is the standard Hamiltonian when expressed in $x$ coordinates,

$$
H(h)=\frac{1}{2} \int_{V_{d}} \mathrm{~d}^{d} x\left(\pi^{2}+\partial_{I} \phi \partial^{I} \phi+\partial_{i} \phi \partial^{i} \phi\right) .
$$

Furthermore, if we define

$$
H_{\iota}(h)=\int_{V_{d}} \mathrm{~d}^{p} x \mathrm{~d}^{d} y \mathcal{H}_{\iota}(h),
$$

using again (4.47), we have in $x$ coordinates

$$
H_{\iota}(h)=-\theta^{i}{ }_{\iota} P_{i}, \quad P_{i}=-\int_{V_{d}} \mathrm{~d}^{d} x \pi \partial_{i} \phi .
$$

It now follows from the usual phase space path integral arguments that, in operator formalism, the path integral weighted by the first order Euclidean action (4.41), corresponds to

$$
\mathrm{Z}_{d, q}\left(\beta, \alpha^{j}, h\right)=\operatorname{Tr} e^{-\beta \hat{H}(h)+i \alpha^{j} \hat{P}_{j}}=\operatorname{Tr} e^{-\beta\left(\hat{H}(h)-i \mu^{j} \hat{P}_{j}\right)},
$$

provided that

$$
\beta=N, \quad \alpha^{j}=\theta^{j}{ }_{\iota} N^{\iota}, \quad \mu^{j}=\theta^{j}{ }_{\iota} \frac{N^{\iota}}{N} .
$$

Note that this result holds for general lattice vectors $e^{a}{ }_{\alpha}$, so that the Iwasawa decomposition (4.45), may also contain a non-trivial rotation matrix. 
We now proceed to directly evaluate the trace (4.51) in the operator approach on the spatial manifold $\mathbb{R}^{p} \times \mathbb{T}^{q}$, with $d=p+q$. As before, in section 4.2 , we start by replacing $\mathbb{R}^{p}$ by $\mathbb{S}_{L_{1}}^{1} \times \cdots \times \mathbb{S}_{L_{p}}^{1}$. An orthonormal basis of functions is

$$
e_{n_{I}, n_{\iota}}(x)=\frac{1}{\sqrt{V_{d}}} e^{i k_{I} x^{I}} e^{2 \pi i n_{\iota} \theta_{i}{ }^{\iota} x^{i}}, \quad k_{I}=\frac{2 \pi n_{I}}{L_{I}}, \quad n_{I} \in \mathbb{Z}^{p}, \quad n_{\alpha} \in \mathbb{Z}^{q+1},
$$

where $V_{d}=V_{d-q} \mathrm{~V}_{q}$. The orthonormality condition is

$$
\begin{aligned}
\left(e_{n_{I}, n_{\iota}}, e_{n_{I}^{\prime}, n_{\iota}^{\prime}}\right) & =\int_{V_{d}} \mathrm{~d}^{d} x e_{n_{I}, n_{\iota}}^{*}(x) e_{n_{I}^{\prime}, n_{\iota}^{\prime}}(x) \\
& =\frac{1}{V_{d}} \int_{V_{d-q}} \mathrm{~d}^{p} x \mathrm{~d}^{q} y \sqrt{h} e^{i\left(k_{I}^{\prime}-k_{I}\right) x^{I}} e^{2 \pi i\left(n_{\iota}^{\prime}-n_{\iota}\right) y^{\iota}}=\prod_{I, \iota} \delta_{n_{I}, n_{I}^{\prime}} \delta_{n_{\iota}, n_{\iota}^{\prime}} .
\end{aligned}
$$

The field $\phi$ and its momentum $\pi$ satisfy the following periodicity conditions along the $x^{i}$ coordinates

$$
\phi\left(x^{I}, x^{i}, x^{d+1}\right)=\phi\left(x^{I}, x^{i}+\theta^{i}, x^{d+1}\right), \quad \pi\left(x^{I}, x^{i}, x^{d+1}\right)=\pi\left(x^{I}, x^{i}+\theta_{\iota}^{i}, x^{d+1}\right),
$$

$\forall \iota=d-q+1, \ldots, d$, and therefore they admit the mode expansions

$$
\phi\left(x^{I}, x^{i}\right)=\sum_{\left(n_{I}, n_{\iota}\right) \in \mathbb{Z}^{d}} \phi_{n_{I}, n_{\iota}} e_{n_{I}, n_{\iota}}(x), \quad \pi\left(x^{I}, x^{i}\right)=\sum_{\left(n_{I}, n_{\iota}\right) \in \mathbb{Z}^{d}} \pi_{n_{I}, n_{\iota}} e_{n_{I}, n_{\iota}}(x),
$$

where the Fourier components satisfy the reality conditions $\phi_{n_{I}, n_{\iota}}=\phi_{-n_{I},-n_{\iota}}^{*}$ and $\pi_{n_{I}, n_{\iota}}=$ $\pi_{-n_{I},-n_{\iota}}^{*}$. Note that, according to the transformation law (4.47), the Fourier components of $\pi\left(x^{I}, y^{\iota}\right)$ are $\sqrt{h} \pi_{n_{I}, n_{\iota}}$. Inserting the mode expansions of $\phi\left(x^{I}, y^{\iota}\right)$ and $\pi\left(x^{I}, y^{\iota}\right)$ and the ansatz for the metric into the Hamiltonian (4.46), we get

$$
H(h)=\frac{1}{2} \sum_{\left(n_{I}, n_{\iota}\right) \in \mathbb{Z}^{d}}\left(\pi_{n_{I}, n_{\iota}} \pi_{n_{I}, n_{\iota}}^{*}+\omega_{k_{i}}^{2} \phi_{n_{I}, n_{\iota}} \phi_{n_{I}, n_{\iota}}^{*}\right),
$$

with

$$
\omega_{k_{i}}=\sqrt{k_{I} k^{I}+(2 \pi)^{2} n_{\iota} h^{\iota \kappa} n_{\kappa}} .
$$

Similarly, using (4.50), the mode expansion of the momenta is

$$
P_{i}=-i \theta_{i}^{\iota} \sum_{\left(n_{I}, n_{\iota}\right) \in \mathbb{Z}^{d}}\left(2 \pi n_{\iota}\right) \pi_{n_{I}, n_{\iota}}^{*} \phi_{n_{I}, n_{\iota}} .
$$

Introducing oscillator variables

$$
a_{n_{I}, n_{\iota}}=\sqrt{\frac{\omega_{k_{i}}}{2}}\left[\phi_{n_{I}, n_{\iota}}+\frac{i}{\omega_{k_{i}}} \pi_{n_{I}, n_{\iota}}\right],
$$

the quantized Hamiltonian without the $\left(n_{I}, n_{\iota}\right)=(0, \ldots, 0)$ mode and the linear momenta are given by

$$
\begin{aligned}
\hat{H}^{\prime}(h) & =\sum_{\left(n_{I}, n_{\iota}\right) \in \mathbb{Z}^{d}}^{\prime} \omega_{k_{i}} \hat{a}_{n_{I}, n_{\iota}}^{\dagger} \hat{a}_{n_{I}, n_{\iota}}+E_{0}^{d, q}(h), \\
\hat{P}_{i} & =\theta_{i}{ }^{\iota} \sum_{\left(n_{i}, n_{\iota}\right) \in \mathbb{Z}^{d}}^{\prime}\left(2 \pi i n_{\iota}\right) \hat{a}_{n_{I}, n_{\iota}}^{\dagger} \hat{a}_{n_{I}, n_{\iota}},
\end{aligned}
$$


where $E_{0}^{d, q}(h)$ is the Casimir energy on $\mathbb{R}^{p} \times \mathbb{T}^{q}$. Introducing $\hat{h}_{\iota \kappa}=\left(\mathrm{V}_{q}\right)^{-\frac{2}{q}} h_{\iota \kappa}$ and $\hat{\theta}^{i}{ }_{\iota}=\left(\mathrm{V}_{q}\right)^{-\frac{1}{q}} \theta^{i}{ }_{\iota}$ with unit determinants, it is explicitly given by

$$
E_{0}^{d, q}(h)=-\frac{1}{2} \frac{V_{d-q}}{\left(\mathrm{~V}_{q}\right)^{\frac{d-q+1}{q}}} \frac{\Gamma\left(\frac{d+1}{2}\right)}{\pi^{\frac{d+1}{2}}} f_{\frac{d+1}{2}}(q ; \hat{h})=-\frac{V_{d-q}}{\left(\mathrm{~V}_{q}\right)^{\frac{d-q+1}{q}}} \xi(d+1, q ; \hat{h}),
$$

and reduces to (3.41) in the case where $q=2$ and where $h$ is diagonal.

We can now evaluate the partition function (4.51),

$$
\begin{aligned}
\mathrm{Z}_{d, q}\left(\beta, \alpha^{j}, h\right) & =\operatorname{Tr} e^{-\beta \hat{H}^{\prime}(h)-i \alpha^{j} \hat{P}_{j}}=e^{-\beta E_{0}^{d, q}(h)} \prod_{\left(n_{I}, n_{\iota}\right) \in \mathbb{Z}^{d} N_{k_{i}}}^{\prime} e^{\left(-\beta \omega_{k_{i}}+2 \pi i \alpha^{j} \theta_{j}{ }^{\iota} n_{\iota}\right) N_{k_{i}}} \\
& =\prod_{\left(n_{I}, n_{\iota}\right) \in \mathbb{Z}^{d}}^{\prime} \frac{e^{-\beta E_{0}^{d, q}(h)}}{1-e^{-\beta \omega_{k_{i}}+2 \pi i \alpha^{j} \theta_{j}^{\iota} n_{\iota}}}
\end{aligned}
$$

Taking the logarithm and turning the sums over $n_{I}$ into integrals gives

$$
\begin{aligned}
& \ln \mathrm{Z}_{d, q}\left(\beta, \alpha^{j}, h\right)=-\beta E_{0}^{d, q}(h) \\
& \quad-\frac{V_{d-q}}{(2 \pi)^{p}} \frac{2 \pi^{\frac{p}{2}}}{\Gamma\left(\frac{p}{2}\right)} \sum_{n_{\iota} \in \mathbb{Z}^{q}} \int_{0}^{\infty} \mathrm{d} k k^{p-1} \ln \left[1-e^{-\beta \sqrt{k^{2}+(2 \pi)^{2} n_{\iota} h^{\iota \kappa} n_{\kappa}}+2 \pi i \alpha^{j} \theta_{j}{ }^{\iota} n_{\iota}}\right] .
\end{aligned}
$$

The rest of the computation proceeds as in section 3.2 with the substitutions $d \rightarrow p+1$, $k_{d}^{2} \rightarrow(2 \pi)^{2} n_{\iota} h^{\iota \kappa} n_{\kappa}$ and $\alpha k_{d} \rightarrow 2 \pi \alpha^{j} \theta_{j} n_{\iota}$. After using the reduplication formula (2.22), the result is

$$
\begin{aligned}
& \ln \mathrm{Z}_{d, q}\left(\beta, \alpha^{j}, h\right)=-\beta E_{0}^{d, q}(h)+\xi(d-q+1) \frac{V_{d-q}}{\beta^{d-q}} \\
& \quad+2 \frac{V_{d-q}}{\beta^{\frac{d-q-1}{2}}} \sum_{n_{\iota} \in \mathbb{Z}^{q} q}^{\prime} \sum_{l \in \mathbb{N}^{*}}\left(\frac{\sqrt{n_{\iota} h^{\iota \kappa} n_{\kappa}}}{l}\right)^{\frac{d-q+1}{2}} K_{\frac{d+1-q}{2}}\left(2 \pi l \beta \sqrt{n_{\iota} h^{\iota \kappa} n_{\kappa}}\right) e^{2 \pi i l n_{\iota} \alpha^{j} \theta_{j}^{\iota}} .
\end{aligned}
$$

When taking into account (4.62), equation (4.65) becomes, in terms of the dimensionless parameter $b=\left(\mathrm{V}_{q}\right)^{-\frac{1}{q}} \beta$,

$$
\begin{aligned}
& \ln \mathrm{Z}_{d, q}\left(\beta, \alpha^{j}, h\right)=\frac{V_{d-q}}{\left(\mathrm{~V}_{q}\right)^{\frac{d-q}{q}}}\left[b \xi(d+1, q ; \hat{h})+\xi(d+1-q) b^{-(d-q)}\right. \\
& \left.+2 b^{-\frac{d-q-1}{2}} \sum_{n_{\iota} \in \mathbb{Z}^{q}}^{\prime} \sum_{l \in \mathbb{N}^{*}}\left(\frac{\sqrt{n_{\iota} \hat{h}^{\iota \kappa} n_{\kappa}}}{l}\right)^{\frac{d+1-q}{2}} K_{\frac{d+1-q}{2}}\left(2 \pi l b \sqrt{n_{\iota} \hat{h}^{\iota \kappa} n_{\kappa}}\right) e^{2 \pi i l n_{\iota} N^{\iota}}\right] \text {. }
\end{aligned}
$$

\section{Remarks.}

(i) For $q=1$, this expression correctly reduces to (3.63) with the identifications $V_{1}=L_{d}$, $b=\tau_{2}$ and $N^{1}=\tau_{1}$. 
(ii) Using the $\mathrm{ADM}$ parametrization, $\mathrm{V}_{q+1}=\mathrm{V}_{q} \beta$ and hence the result for the partition function in (4.22) reads

$$
\ln \mathrm{Z}_{\mathrm{d}, \mathrm{q}}\left(\beta, \alpha^{j}, h\right)=\frac{V_{d-q}}{\left(\mathrm{~V}_{q} \beta\right)^{\frac{d-q}{q+1}}} \xi\left(d+1, q+1 ; \hat{g}^{\mathrm{ADM}}\right),
$$

where

$$
\begin{gathered}
\hat{g}_{\alpha \beta}^{\mathrm{ADM}}=b^{-\frac{2}{q+1}}\left(\begin{array}{cc}
\hat{h}_{\iota \kappa} & \hat{h}_{\kappa \lambda} N^{\lambda} \\
\hat{h}_{\iota \lambda} N^{\lambda} & b^{2}+\hat{h}_{\lambda \xi} N^{\lambda} N^{\xi}
\end{array}\right), \\
\hat{g}_{\mathrm{ADM}}^{\alpha \beta}=b^{-\frac{2 q}{q+1}}\left(\begin{array}{cc}
b^{2} \hat{h}^{\iota \kappa}+N^{\iota} N^{\kappa} & -N^{\kappa} \\
-N^{\iota} & 1
\end{array}\right) .
\end{gathered}
$$

Comparing (4.67) and (4.66), suggests that the Fourier transform of the completed $\mathrm{SL}(n, \mathbb{Z})$ Eisenstein series for an $n$-dimensional metric $\hat{g}_{\alpha \beta}^{\mathrm{ADM}}$ with unit determinant parametrized in $\mathrm{ADM}$ form is

$$
\begin{aligned}
\xi\left(z, n ; \hat{g}^{\mathrm{ADM}}\right)= & b^{\frac{z}{n}} \xi(z, n-1 ; \hat{h})+b^{\frac{(n-z)(n-1)}{n}} \xi(z-n+1) \\
& +2 b^{\frac{2(z-n)-(z-n-1) n}{2 n}} \sum_{n_{\iota} \in \mathbb{Z}^{n-1}}^{\prime} \sum_{l \in \mathbb{N}^{*}}\left(\frac{\sqrt{n_{\iota} \hat{h}^{\iota \kappa} n_{\kappa}}}{l}\right)^{\frac{z-n+1}{2}} \\
& K_{\frac{z-n+1}{2}}\left(2 \pi l b \sqrt{n_{\iota} \hat{h}^{\iota \kappa} n_{\kappa}}\right) e^{2 \pi i l n_{\iota} N^{\iota}}
\end{aligned}
$$

in the sense that the equality of (4.67) and (4.66) corresponds to the case $z=d+1$ and $n=q+1$.

(iii) At low temperature/small box $b \gg 1$, the leading term in the expansion to the partition function is directly related to the Casimir energy. The leading correction is the contribution of the modes with spatial frequencies $n_{\iota}=0$. It coincides with the black body result (2.24) of a massless scalar field in $d-q$ spatial dimensions. It is independent of $\mathrm{V}_{q}$ and does not contribute to the Casimir pressure,

$$
\mathrm{p}_{d, q}\left(\beta, \alpha^{j}, h\right)=\frac{1}{V_{d-q}} \frac{\partial\left(\beta^{-1} \ln \mathrm{Z}_{d, q}\left(\beta, \alpha^{j}, h\right)\right)}{\partial \mathrm{V}_{q}} .
$$

The asymptotic behavior of the modified Bessel functions in (3.55) implies that all other terms are exponentially suppressed. The low-temperature/small box expansion of the Casimir pressure is thus,

$$
\mathrm{p}_{d, q}^{\text {low }}\left(\beta, \alpha^{j}, h\right)=-\frac{d-q+1}{q} \frac{1}{\mathrm{~V}_{q}^{\frac{d+1}{q}}} \xi(d+1, q ; \hat{h})+\ldots,
$$

where the dots denote exponentially suppressed terms. For the low-temperature/small box expansion of the entropy

$$
\mathrm{S}_{d, q}\left(\beta, \alpha^{j}, h\right)=\left(1-\beta \partial_{\beta}\right) \ln \mathrm{Z}_{d, q}\left(\beta, \alpha^{j}, h\right),
$$


on the other hand, the first term in (4.66) proportional to the Casimir energy drops out since it is linear in $\beta$, while the leading term now comes from the lower dimensional scalar field, i.e., the modes with $n_{\iota}=0$,

$$
\mathrm{S}_{d, q}^{\text {low }}\left(\beta, \alpha^{j}, h\right)=(d+1-q) \xi(d+1-q) \frac{V_{d-q}}{\beta^{d-q}}+\ldots
$$

Since the leading terms in the low temperature expansions (4.72), (4.74) do not depend on $\alpha^{j}$, they are the same when starting from $\ln Z_{d, q}\left(\beta, \mu^{j}, h\right)$ with $\alpha^{j}=\beta \mu^{j}$.

(iv) In order to discuss the high temperature/large box limit, we start by using the reflection formula (4.21) on the partition function in (4.22) with a metric in ADM parametrization gives

$$
\ln Z_{d, q}\left(g^{\mathrm{ADM}}\right)=\frac{V_{d-q}}{\left(\beta \mathrm{V}_{q}\right)^{\frac{d-q}{q+1}}} \xi\left(q-d, q+1 ; \hat{g}_{\mathrm{ADM}}^{-1}\right) .
$$

For the Fourier transform in (4.70), we need to parametrize $\hat{g}_{\mathrm{ADM}}^{-1}$ in ADM form of a metric with unit determinant rather than the ADM form of an inverse metric with unit determinant. This implies that one now parametrizes the frame and co-frame as

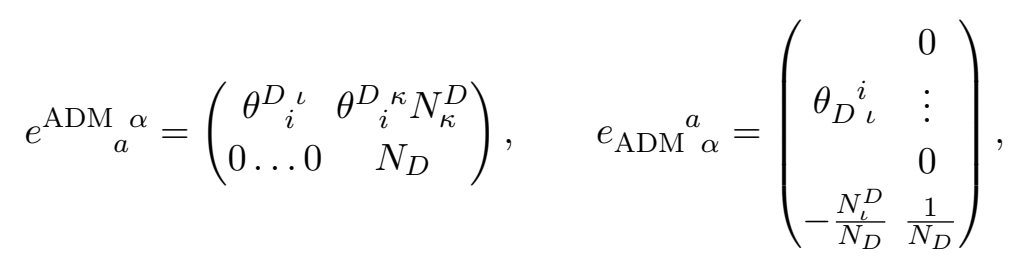

and one parametrizes the associated metric and its inverse as

$$
g_{\mathrm{ADM}}^{\alpha \beta}=\left(\begin{array}{cc}
h_{D}{ }^{\iota \kappa} & N_{D}^{\kappa} \\
N_{D}^{\iota} & N_{D}^{2}+N_{\lambda}^{D} N_{D}^{\lambda}
\end{array}\right), \quad g_{\alpha \beta}^{\mathrm{ADM}}=\left(\begin{array}{cc}
h^{D}{ }_{\iota \kappa}+\frac{N_{\iota}^{D} N_{\kappa}^{D}}{N_{D}^{2}} & -\frac{N_{\kappa}^{D}}{N_{D}^{2}} \\
-\frac{N_{l}^{D}}{N_{D}^{2}} & \frac{1}{N_{D}^{2}}
\end{array}\right),
$$

with $N_{D}^{\iota}=h_{D}{ }^{\iota \kappa} N_{\kappa}^{D}$ and $h_{D}{ }^{\iota \kappa}=\theta^{D}{ }_{i}{ }^{\iota} \delta^{i j} \theta^{D}{ }_{j}{ }^{\kappa}$. Comparing to the usual ADM parametrization in (4.39), it follows that the dual and standard ADM parameters are related as

$$
\begin{aligned}
& h_{D}^{\iota \kappa}=h^{\iota \kappa}+\frac{N^{\iota} N^{\kappa}}{N^{2}}, \quad \frac{1}{N_{D}^{2}}=N^{2}+N_{\lambda} N^{\lambda}, \quad N_{D}^{\iota}=-\frac{N^{\iota}}{N^{2}}, \\
& h_{\iota \kappa}^{D}=h_{\iota \kappa}-\frac{N_{\iota} N_{\kappa}}{N^{2}+N_{\lambda} N^{\lambda}},
\end{aligned}
$$

and then also as,

$$
\frac{1}{N^{2}}=N_{D}^{2}+N_{\lambda}^{D} N_{D}^{\lambda}, \quad N_{\iota}=-\frac{N_{\iota}^{D}}{N_{D}^{2}}, \quad h_{\iota \kappa}=h_{\iota \kappa}^{D}+\frac{N_{\iota}^{D} N_{\kappa}^{D}}{N_{D}^{2}} .
$$

From (4.77), it follows that the determinant of $g_{\alpha \beta}^{\mathrm{ADM}}$ which is $N^{2} \mathrm{~V}_{q}^{2}$ in the standard parametrization, is given by $\operatorname{det} g^{\mathrm{ADM}}=N_{D}^{-2} \operatorname{det} h^{D} \equiv N_{D}^{-2}\left(\mathrm{~V}_{q}^{D}\right)^{2}$ in the dual parametrization. As a consequence, $\mathrm{V}_{q}^{D}=\mathrm{V}_{q} N N_{D}$, and using (4.78),

$$
\mathrm{V}_{q}^{D}=\mathrm{V}_{q} \frac{N}{\sqrt{N^{2}+N_{\lambda} N^{\lambda}}}
$$


Introducing the adimensional parameter $b_{D}=N_{D}\left(\mathrm{~V}_{q}^{D}\right)^{\frac{1}{q}}$, we have for the normalized metric,

$$
\begin{aligned}
& \hat{g}_{\alpha \beta}^{\mathrm{ADM}}=\left(b_{D}\right)^{\frac{-2 q}{q+1}}\left(\begin{array}{cc}
b_{D}^{2} \hat{h}_{\iota \kappa}^{D}+N_{\iota}^{D} N_{\kappa}^{D} & -N_{\kappa}^{D} \\
-N_{\iota}^{D} & 1
\end{array}\right), \\
& \hat{g}_{\mathrm{ADM}}^{\alpha \beta}=\left(b_{D}\right)^{-\frac{2}{q+1}}\left(\begin{array}{cc}
\hat{h}_{D}^{\iota \kappa} & N_{D}^{\kappa} \\
N_{D}^{\iota} & b_{D}^{2}+\hat{h}_{\iota \kappa}^{D} N_{D}^{\iota} N_{D}^{\kappa}
\end{array}\right) .
\end{aligned}
$$

In terms of the dual chemical potentials $\mu_{D}^{j}=\frac{\theta_{D}{ }^{j}{ }^{\imath} N_{D}^{\iota}}{N_{D}}=\frac{\alpha_{D}^{j}}{N_{D}}$, it follows that equations (4.78) are equivalent to

$$
\begin{aligned}
& b_{D}=\frac{1}{b} \frac{1}{\left(1+\mu_{i} \mu^{i}\right)^{\frac{q+1}{2 q}}}, \quad \hat{\theta}_{D}^{j}{ }_{D^{\iota}} b_{D} \mu_{j}^{D}=-\frac{1}{b} \frac{1}{\left(1+\mu_{i} \mu^{i}\right)} \hat{\theta}^{j}{ }_{\iota} \mu_{j}, \\
& \hat{h}_{\iota \kappa}^{D}=\left(1+\mu_{i} \mu^{i}\right)^{\frac{1}{q}}\left(\hat{h}_{\iota \kappa}-\hat{\theta}^{j}{ }_{\iota} \hat{\theta}^{k}{ }_{\kappa} \frac{\mu_{j} \mu_{k}}{1+\mu_{i} \mu^{i}}\right),
\end{aligned}
$$

and that $\mathrm{V}_{q}^{D}=\mathrm{V}_{q}\left(1+\mu_{i} \mu^{i}\right)^{-\frac{1}{2}}$. Note in particular that in the case $q=1$, the first two equations reduce to the $S$ transformation of (3.65) while the last equation reduces to a trivial identity.

We are now in a position to apply the Fourier transform (4.70) at $z=q-d, n=q+1$, to $(4.75)$,

$$
\begin{aligned}
& \ln Z_{d, q}\left(g^{\mathrm{ADM}}\right)=\frac{V_{d-q}}{\left(\beta \mathrm{V}_{q}\right)^{\frac{d-q}{q+1}}}\left[b_{D}^{\frac{q-d}{q+1}} \xi\left(q-d, q ; \hat{h}_{D}^{-1}\right)+b_{D}^{\frac{q(d+1)}{q+1}} \xi(-d)\right. \\
& +2 b_{D}^{\frac{d q+2 q-d}{2(q+1)}} \sum_{m^{\iota} \in \mathbb{Z}^{q}}^{\prime} \sum_{l \in \mathbb{N}^{*}}\left(\frac{\sqrt{m^{\iota} \hat{h}_{\iota \kappa}^{D} m^{\kappa}}}{l}\right)^{-\frac{d}{2}} K_{-\frac{d}{2}}\left(2 \pi l b_{D} \sqrt{m^{\iota} \hat{h}_{\iota \kappa}^{D} m^{\kappa}}\right) e^{\left.2 \pi i l m^{\iota} N_{\iota}^{D}\right]},
\end{aligned}
$$

or, after using the reflection formulas on the first two terms, $K_{\nu}(z)=K_{-\nu}(z)$, and simplifying,

$$
\begin{aligned}
& \ln Z_{d, q}\left(g^{\mathrm{ADM}}\right)=\frac{V_{d-q}}{\left(\mathrm{~V}_{q}^{D}\right)^{\frac{d-q}{q}}\left[b_{D}^{d} \xi(d+1)+\xi\left(d, q ; \hat{h}_{D}\right)\right.} \\
& \left.+2 b_{D}^{\frac{d}{2}} \sum_{m^{\iota} \in \mathbb{Z}^{q}}^{\prime} \sum_{l \in \mathbb{N}^{*}}\left(\frac{\sqrt{m^{\iota} \hat{h}_{\iota \kappa}^{D} m^{\kappa}}}{l}\right)^{-\frac{d}{2}} K_{\frac{d}{2}}\left(2 \pi l b_{D} \sqrt{m^{\iota} \hat{h}_{\iota \kappa}^{D} m^{\kappa}}\right) e^{2 \pi i l m^{\iota} N_{\iota}^{D}}\right],
\end{aligned}
$$

where $N_{\iota}^{D}=\hat{\theta}_{D \iota}^{i} b_{D} \mu_{i}^{D}$. The high-temperature/large volume limit is now defined by $b \ll 1$ so that $b_{D} \sim b^{-1} \gg 1$. This agrees with the discussion after (3.67) in the case $q=1$. Furthermore, in that case, the appropriate powers of $\left(1+\mu^{2}\right)$ that relate the high and low temperature expansions may be obtained from the definitions of $b_{D}$ and 
$\mathrm{V}_{q}^{D}$ above. The asymptotic behaviour of the Bessel function in (3.55) implies that all the terms appearing in the sums in (4.85) are exponentially suppressed. We thus get in the high-temperature/large volume limit,

$$
\begin{aligned}
\ln Z_{d, q}^{\text {high }}\left(\beta, \mu^{i}, h\right)= & \frac{V_{d-q} \mathrm{~V}_{q}}{\beta^{d}} \xi(d+1)\left(1+\mu_{i} \mu^{i}\right)^{-\frac{d+1}{2}} \\
& +\frac{V_{d-q}}{\left(\mathrm{~V}_{q}\right)^{\frac{d-q}{q}}}\left(1+\mu_{i} \mu^{i}\right)^{\frac{d-q}{2 q}} \xi\left(d, q ; \hat{h}_{D}\right)+\ldots
\end{aligned}
$$

The first term of this expression is the black body term, suitably corrected by the coupling of the linear momenta, while the second reduces, in the $q=1$ case, to the second of (3.69). Note that, while in the $q=1$ case, the high-temperature limit of the partition function could be simply obtained by using modular covariance (3.15) of $\ln \mathcal{Z}_{d}(\tau, \bar{\tau})$ under the S-transformation $\tau^{\prime}=-\frac{1}{\tau}$ as shown in (3.67) and (3.69), in the case $q>1$, we had to use a construction involving the dual parametrization in this derivation here. The associated expansion of the Casimir pressure and the entropy are

$$
\begin{aligned}
p_{d, q}^{\text {high }}\left(\beta, \mu^{j}, h\right)= & \frac{\xi(d+1)}{\beta^{d+1}}\left(1+\mu_{i} \mu^{i}\right)^{-\frac{d+1}{2}} \\
& -\frac{(d-q) \xi\left(d, q ; \hat{h}_{D}\right)}{q \beta\left(\mathrm{V}_{q}\right)^{\frac{d}{q}}}\left(1+\mu_{i} \mu^{i}\right)^{\frac{d-q}{2 q}}+\ldots
\end{aligned}
$$

and

$$
\begin{aligned}
S_{d, q}^{\mathrm{high}}\left(\beta, \mu^{j}, h\right)= & \frac{V_{d-q} \mathrm{~V}_{q}}{\beta^{d}}(d+1) \xi(d+1)\left(1+\mu_{i} \mu^{i}\right)^{-\frac{d+1}{2}} \\
& +\frac{V_{d-q}}{\left(\mathrm{~V}_{q}\right)^{\frac{d-q}{q}}}\left(1+\mu_{i} \mu^{i}\right)^{\frac{d-q}{2 q}} \xi\left(d, q ; \hat{h}_{D}\right)+\ldots,
\end{aligned}
$$

respectively. The associated asymptotic micro-canonical density of states is now

$$
\ln \rho_{d}\left(E, \mu^{j}, h\right) \approx(d+1)\left(\frac{E}{d}\right)^{\frac{d}{d+1}}\left[\xi(d+1) V_{d-q} \mathrm{~V}_{q}\right]^{\frac{1}{d+1}} \frac{1}{\sqrt{1+\mu_{i} \mu^{i}}},
$$

that can be also written as

$$
\ln \rho_{d}\left(E, \mu^{j}, h\right) \approx(d+1)\left[\frac{E\left(\mathrm{~V}_{q}\right)^{\frac{1}{q}}}{d}\right]^{\frac{d}{d+1}}\left(\frac{\pi c_{d, q}}{6}\right)^{\frac{1}{d+1}} \frac{1}{\sqrt{1+\mu_{i} \mu^{i}}},
$$

where

$$
c_{d, q}=\frac{6 \xi(d+1)}{\pi} \frac{V_{d-q}}{\left(\mathrm{~V}_{q}\right)^{\frac{d-q}{q}}}
$$

reduces to (3.28) for $q=1$. Note however that, unlike what happens in the $q=1$ case, here $c_{d, q}$ cannot be related to the Casimir energy $E_{0}^{d, q}(h)$. 
(v) The partition function contains additional integrated observables through the dependence of the Hamiltonian on the parameters encoded in $h^{\iota \kappa}$. Defining,

$$
\begin{aligned}
{ }^{(q)} \mathcal{T}_{\iota \kappa} & =\frac{2}{\sqrt{h}} \frac{\partial S_{H}^{E}\left[\phi, \pi ; g^{\mathrm{ADM}}\right]}{\partial h^{\iota \kappa}} \\
& =\int_{V_{d-q}} \mathrm{~d}^{p} x \mathrm{~d}^{q+1} y N\left[\partial_{\iota} \phi \partial_{\kappa} \phi-\frac{1}{2} h_{\iota \kappa}\left(-\frac{1}{h} \pi^{2}+h^{\lambda \xi} \partial_{\lambda} \phi \partial_{\xi} \phi+\partial_{I} \phi \partial^{I} \phi\right)\right],
\end{aligned}
$$

which correspond to

$$
\begin{aligned}
{ }^{(q)} \mathcal{T}_{i k} & =\sqrt{h} \theta_{i}{ }^{\iota} \theta_{k}{ }^{\kappa(q)} \mathcal{T}_{\iota \kappa} \\
& =\int_{V_{d+1}} \mathrm{~d}^{d+1} x\left[\partial_{i} \phi \partial_{k} \phi-\frac{1}{2} \delta_{i k}\left(-\pi^{2}+\partial_{i} \phi \partial^{i} \phi+\partial_{I} \phi \partial^{I} \phi\right)\right],
\end{aligned}
$$

in $x$ coordinates, it follows that

$$
\left\langle{ }^{(q)} \widehat{\mathcal{T}}_{\iota \kappa}\right\rangle=-\frac{2}{\sqrt{h}} \frac{\partial}{\partial h^{\iota \kappa}} \ln Z_{d, q}(g) .
$$

As in the covariant case, one may consider instead the variable $v$ defined by $h_{\iota \kappa}=v \hat{h}_{\iota \kappa}$ with $\mathrm{V}_{q}=\sqrt{h}=(v)^{\frac{q}{2}}, N=b\left(\mathrm{~V}_{q}\right)^{\frac{1}{q}}=b(v)^{\frac{1}{2}}$, and the integrated observables

$$
{ }^{(q)} \mathcal{D}_{\iota \kappa}=\int \mathrm{d}^{p} x \mathrm{~d}^{q+1} y \partial_{\iota} \phi \partial_{\kappa} \phi,
$$

such that

$$
\begin{aligned}
\frac{\partial S_{H}^{E}\left[\phi, \pi ; g^{\mathrm{ADM}}\right]}{\partial \hat{h}_{\iota \kappa}} & =-\frac{\sqrt{h}}{2} v^{(q)} J_{\xi \lambda}{ }^{\iota \kappa(q)} \mathcal{T}^{\xi \lambda}=-\frac{1}{2}(v)^{\frac{q-1}{2}} b^{(q)} \hat{J}^{\iota \kappa \xi \lambda(q)} \mathcal{D}_{\xi \lambda}, \\
v \frac{\partial S_{H}^{E}\left[\phi, \pi ; g^{\mathrm{ADM}}\right]}{\partial v} & =-\frac{1}{2}(v)^{\frac{q}{2}} h^{\iota \kappa(q)} \mathcal{T}_{\iota \kappa} .
\end{aligned}
$$

Notice that the last observable is directly related to the Casimir pressure. It then follows from (A.18) that

$$
h^{\iota \kappa}\left\langle{ }^{(q)} \hat{\mathcal{T}}_{\iota \kappa}\right\rangle=(q-d) \frac{1}{\mathrm{~V}_{q}} \ln Z_{d, q}\left(g^{\mathrm{ADM}}\right) .
$$

More generally, the variables of the Hamiltonian formalism may be chosen as $v$, $\hat{h}_{\iota \kappa}, N^{\iota}, b$, while those of the Lagrangian formalism as $t, \hat{g}_{\alpha \beta}$. When using the ADM parametrization (4.68) together with $t=v(b)^{\frac{2}{q+1}}$, the equivalence of the Lagrangian and Hamiltonian path integrals implies not only

$$
g^{\alpha \beta}\left\langle\hat{\mathcal{T}}_{\alpha \beta}\right\rangle=\frac{1}{(v)^{\frac{1}{2} b}} h^{\iota \kappa}\left\langle{ }^{(q)} \hat{\mathcal{T}}_{\iota \kappa}\right\rangle,
$$

in agreement with (4.97) and (4.38), but yields more generally the relations between Lagrangian and Hamiltonian observables inside correlation functions. In particular, when expressed in terms of Hamiltonian observables, the relation (4.37) will be equivalent to applying the $\mathrm{ADM}$ form of the $\mathrm{SL}(q+1)$ Laplacian in (A.28) to (4.67). 
Indeed, to use this Laplacian, it is the spacetime volume $\mathrm{V}_{q+1}$ that is kept fixed, so that this Laplacian acts on (4.67) with fixed pre-factor $V_{d-q}\left(\mathrm{~V}_{q+1}\right)^{-\frac{d-q}{q+1}} \xi\left(d+1, q+1 ; g^{\mathrm{ADM}}\right)$ and the powers of $b$ are those in (4.70) with $z=d+1, n=q+1$ rather than those in (4.66).

(vi) In the case $q=1$, the issue of modular covariance of $\ln \mathcal{Z}_{d}(\tau, \bar{\tau})$ in (3.13) versus $\operatorname{SL}(2, \mathbb{Z})$ invariance of $\ln Z_{d}(g)$ in (4.22) can be clarified when working with homogeneous coordinates. A general vielbein characterizing $\mathbb{T}^{2}$ and its Iwasawa decomposition may be written in terms of two complex variables $z_{1}=x_{1}+i y_{1}, z_{2}=x_{2}+i y_{2}$ as

$$
\begin{aligned}
e^{a}{ }_{\alpha} & =\left(\begin{array}{ll}
x_{1} & x_{2} \\
y_{1} & y_{2}
\end{array}\right) \\
& =\left(\begin{array}{cc}
\frac{x_{1}}{\left|z_{1}\right|} & -\frac{y_{1}}{\left|z_{1}\right|} \\
y_{1} & \frac{x_{1}}{\left|z_{1}\right|}
\end{array}\right) \sqrt{\mathfrak{I m}\left(\bar{z}_{1} z_{2}\right)}\left(\begin{array}{cc}
\frac{\left|z_{1}\right|}{\sqrt{\mathfrak{I m}\left(\bar{z}_{1} z_{2}\right)}} & 0 \\
0 & \frac{\sqrt{\mathfrak{I m}\left(\bar{z}_{1} z_{2}\right)}}{\left|z_{1}\right|}
\end{array}\right)\left(\begin{array}{cc}
1 & \frac{\mathfrak{R e}\left(\bar{z}_{1} z_{2}\right)}{\left|z_{1}\right|^{2}} \\
0 & 1
\end{array}\right),
\end{aligned}
$$

where we assume $\mathfrak{I m}\left(\bar{z}_{1} z_{2}\right)>0$. This gives

$$
g_{\alpha \beta}=\left(\begin{array}{cc}
\left|z_{1}\right|^{2} & \mathfrak{R e}\left(\bar{z}_{1} z_{2}\right) \\
\mathfrak{R e}\left(\bar{z}_{1} z_{2}\right) & \left|z_{2}\right|^{2}
\end{array}\right), \quad g^{\alpha \beta}=\frac{1}{\left(\mathfrak{I m}\left(\bar{z}_{1} z_{2}\right)\right)^{2}}\left(\begin{array}{cc}
\left|z_{2}\right|^{2} & -\mathfrak{R e}\left(\bar{z}_{1} z_{2}\right) \\
-\mathfrak{R e}\left(\bar{z}_{1} z_{2}\right) & \left|z_{1}\right|^{2}
\end{array}\right),
$$

where the relation

$$
\left|z_{2}\right|^{2}=\frac{\left(\mathfrak{R e}\left(\bar{z}_{1} z_{2}\right)\right)^{2}+\left(\mathfrak{I m}\left(\bar{z}_{1} z_{2}\right)\right)^{2}}{\left|z_{1}\right|^{2}}
$$

holds. Note that we have added the matrix element $y_{1}=e_{d}^{d+1}$, which was absent in (4.30), so that the earlier discussion is recovered when $x_{1}=L_{d}, y_{1}=0, x_{2}=\alpha$, $y_{2}=\beta$.

Under an $\mathrm{SL}(2, \mathbb{Z})$ transformation as in $(4.33),\left(e^{\prime}\right)^{a}{ }_{\alpha}=e^{a}{ }_{\beta} S_{\alpha}{ }^{\beta}$, we have

$$
\left(\begin{array}{ll}
x_{1}^{\prime} & x_{2}^{\prime} \\
y_{1}^{\prime} & y_{2}^{\prime}
\end{array}\right)=\left(\begin{array}{ll}
d x_{1}+c x_{2} & b x_{1}+a x_{2} \\
d y_{1}+c y_{2} & b y_{1}+a y_{2}
\end{array}\right),
$$

while the modular parameter, which according to (4.32) is now given by

$$
\tau=\frac{z_{2}}{z_{1}}
$$

continues to transform as in (1.2). The ADM form of the metric (4.100) is obtained from the change of variables

$$
N=\frac{\mathfrak{I m}\left(\bar{z}_{1} z_{2}\right)}{\left|z_{1}\right|}, \quad N^{1}=\frac{\mathfrak{R e}\left(\bar{z}_{1} z_{2}\right)}{\left|z_{1}\right|^{2}}, \quad h_{11}=N_{(1)}^{2}=\left|z_{1}\right|^{2} .
$$

When using (4.52), it follows that temperature and the chemical potential are given in terms of the parameters $z_{1}, z_{2}$ characterizing the torus by the non-linear relations

$$
\beta=N=\frac{\mathfrak{I m}\left(\bar{z}_{1} z_{2}\right)}{\left|z_{1}\right|}, \quad \alpha=\sqrt{h_{11}} N^{1}=\frac{\mathfrak{R e}\left(\bar{z}_{1} z_{2}\right)}{\left|z_{1}\right|} .
$$


For notational convenience, we also introduce

$$
\gamma=\sqrt{h_{11}}=\left|z_{1}\right|
$$

In these terms,

$$
H(\gamma)=\frac{1}{2} \int_{V_{d-1}} \mathrm{~d}^{p} x \mathrm{~d}^{2} y\left[\frac{1}{\gamma}\left(\pi^{2}+\partial_{1} \phi \partial_{1} \phi\right)+\gamma \partial_{I} \phi \partial^{I} \phi\right],
$$

and

$$
{ }^{(1)} \mathcal{T}_{11}=-\frac{1}{2} \int_{V_{d-1}} \mathrm{~d}^{p} x \mathrm{~d}^{2} y \beta\left[\pi^{2}+\partial_{1} \phi \partial_{1} \phi-\gamma^{2} \partial_{I} \phi \partial^{I} \phi\right] .
$$

Writing

$$
\mathrm{Z}_{\mathrm{d}}(\beta, \alpha, \gamma)=\operatorname{Tr} e^{-\beta \hat{H}(\gamma)+i \alpha P_{d}},
$$

it follows from (4.67) that

$$
\ln \mathrm{Z}_{\mathrm{d}, 1}(\beta, \alpha, \gamma)=\frac{V_{d-1}}{(\gamma \beta)^{\frac{d-1}{2}}} \xi\left(d+1,2 ; \hat{g}^{\mathrm{ADM}}\right),
$$

where the ADM metric with unit determinant is expressed in terms of the chemical potentials $\beta, \alpha, \gamma$ as

$$
\hat{g}_{\alpha \beta}^{\mathrm{ADM}}=\left(\begin{array}{cc}
\frac{\gamma}{\beta} & \frac{\alpha}{\beta} \\
\frac{\alpha}{\beta} & \frac{\beta}{\gamma}+\frac{\alpha^{2}}{\beta \gamma}
\end{array}\right) .
$$

The transformation laws of the chemical potentials under $\operatorname{SL}(2, \mathbb{Z})$ transformation can be obtained from those of $z_{1}, z_{2}$ in (4.102) and are explicitly given by

$$
\begin{aligned}
\gamma^{\prime} & =\sqrt{d^{2} \gamma^{2}+2 c d \gamma \alpha+c^{2}\left(\alpha^{2}+\beta^{2}\right)}, \\
\beta^{\prime} & =\frac{\beta \gamma}{\sqrt{d^{2} \gamma^{2}+2 c d \gamma \alpha+c^{2}\left(\alpha^{2}+\beta^{2}\right)}}, \\
\alpha^{\prime} & =\frac{d b \gamma^{2}+a c\left(\alpha^{2}+\beta^{2}\right)+(a d+b c) \alpha \gamma}{\sqrt{d^{2} \gamma^{2}+2 c d \gamma \alpha+c^{2}\left(\alpha^{2}+\beta^{2}\right)}} .
\end{aligned}
$$

It follows that $(4.110)$ is fully $\operatorname{SL}(2, \mathbb{Z})$ invariant under these transformations, whereas it is covariant under modular transformations of the modular parameter $\tau$ alone.

Finally, we note that in terms of the modular parameter (4.103), the ADM metric with unit determinant keeps the same form as in (4.30),

$$
\hat{g}_{\alpha \beta}^{\mathrm{ADM}}=\frac{1}{\tau_{2}}\left(\begin{array}{cc}
1 & \tau_{1} \\
\tau_{1} & |\tau|^{2}
\end{array}\right) .
$$

In the particular case where $x_{1}=L_{d}=\gamma, y_{1}=0, x_{2}=\alpha, y_{2}=\beta$, the partition function in (4.110), when expressed in terms of $\tau$ and $\bar{\tau}$, reduces to (3.13) as it should. 


\subsection{Worldline approach}

In the world-line approach, one considers a particle on the skewed Euclidean torus $\mathbb{T}^{q+1}$. The Hamiltonian is

$$
\hat{\mathrm{H}}_{\mathbb{T}^{q+1}}=\delta^{a b} \hat{p}_{a} \hat{p}_{b}=g^{\alpha \beta} \hat{p}_{\alpha} \hat{p}_{\beta},
$$

$\hat{p}_{\alpha}=-i \partial_{\alpha}$. The basis of eigenfunctions of the Laplacian on $\mathbb{T}^{q+1}$ are the $e_{n_{\alpha}}(y)=\langle y \mid p\rangle$ given in (4.10), where $|y\rangle \equiv\left|y^{\alpha}\right\rangle$ and $|p\rangle \equiv\left|p_{\alpha}\right\rangle$ with $p_{\alpha}=2 \pi n_{\alpha}$. The heat kernel is

$$
\begin{aligned}
& K_{\mathbb{T}^{q+1}}\left(y^{\prime \alpha}, y^{\alpha} ; t\right)=\left\langle y^{\prime}\left|e^{-t \hat{\mathrm{H}}_{\mathbb{T}^{q+1}}}\right| y\right\rangle=\sum_{n_{\alpha} \in \mathbb{Z}^{d+1}} \sum_{n_{\alpha}^{\prime} \in \mathbb{Z}^{d+1}}\left\langle y^{\prime} \mid p^{\prime}\right\rangle\left\langle p^{\prime}\left|e^{-t \hat{\mathrm{H}}_{\mathbb{T}^{q+1}}}\right| p\right\rangle\langle p \mid y\rangle \\
& \quad=\frac{1}{\mathrm{~V}_{q+1}} \sum_{n_{\alpha} \in \mathbb{Z}^{d+1}} e^{2 \pi i n_{\alpha}\left(y^{\prime \alpha}-y^{\alpha}\right)} e^{-t(2 \pi)^{2} g^{\alpha \beta} n_{\alpha} n_{\beta}}=\frac{1}{\mathrm{~V}_{q+1}} \vartheta_{d+1}\left(y^{\prime \alpha}-y^{\alpha} \mid i t 4 \pi g^{-1}\right),
\end{aligned}
$$

where a resolution of the identity in terms of momentum eigenstates has been used and the result has been expressed in terms of the Riemann theta function in (3.88). Including also the contribution of the large $d-q$ dimensions, the heat kernel on $\mathbb{R}^{p} \times \mathbb{T}^{q+1}$ is

$$
\begin{aligned}
& K_{\mathbb{R}^{p} \times \mathbb{T}^{q+1}}\left(x^{\prime I}, y^{\prime \alpha}, x^{I}, y^{\alpha} ; t\right) \\
& =\frac{1}{(4 \pi t)^{\frac{p}{2}} \mathrm{~V}_{q+1}} e^{-\frac{1}{4 t}\left(x_{I}^{\prime}-x_{I}\right)\left(x^{\prime I}-x^{I}\right)} \vartheta_{q+1}\left(y^{\prime \alpha}-y^{\alpha} \mid i t 4 \pi g^{-1}\right) .
\end{aligned}
$$

Its trace reduces to

$$
\operatorname{Tr} e^{-t \hat{\mathrm{H}}_{\mathbb{R}^{p} \times \mathbb{T}^{q+1}}}=\frac{V_{p}}{(4 \pi t)^{\frac{d-q}{2}}} \sum_{n_{\alpha} \in \mathbb{Z}^{q+1}} e^{-t(2 \pi)^{2} g^{\alpha \beta} n_{\alpha} n_{\beta}}=\frac{V_{p}}{(4 \pi t)^{\frac{d-q}{2}}} \theta_{q+1}\left(t 4 \pi g^{-1}\right),
$$

while the partition function becomes

$$
\begin{aligned}
& \ln Z_{d, q}(g)=\frac{V_{d-q}}{2} \sum_{n_{\alpha} \in \mathbb{Z}^{q+1}}^{\prime} \int_{0}^{\infty} \frac{\mathrm{d} t}{t} \frac{1}{(4 \pi t)^{\frac{d-q}{2}}} e^{-t(2 \pi)^{2} g^{\alpha \beta} n_{\alpha} n_{\beta}} \\
& =\frac{1}{2} \frac{V_{d-q}}{\left(\mathrm{~V}_{q+1}\right)^{\frac{d-q}{q+1}}} \int_{0}^{\infty} \mathrm{d} t t^{\frac{q-d}{2}-1}\left(\theta_{q+1}\left(t \hat{g}^{-1}\right)-1\right) \text {. }
\end{aligned}
$$

Alternatively, a path integral computation for the heat kernel on $\mathbb{T}^{q+1}$ that includes paths winding around the cycles of the torus yields

$$
K_{\mathbb{T}^{q+1}}\left(y^{\prime \alpha}, y^{\alpha} ; t\right)=\frac{1}{(4 \pi t)^{\frac{q+1}{2}}} \sum_{m^{\alpha} \in \mathbb{Z}^{q+1}} e^{-\frac{1}{4 t} g_{\alpha \beta}\left(y^{\prime \alpha}-y^{\alpha}+m^{\alpha}\right)\left(y^{\prime \beta}-y^{\beta}+m^{\beta}\right)},
$$

which can be shown to be equivalent to (4.115) by using the Poisson summation formula (2.56). For $\mathbb{R}^{p} \times \mathbb{T}^{q+1}$, one finds now

$$
\frac{K_{\mathbb{R}^{p} \times \mathbb{T}^{q+1}}\left(x^{\prime I}, y^{\prime \alpha}, x^{I}, y^{\alpha} ; t\right)=}{\sum_{m^{\alpha} \in \mathbb{Z}^{q+1}} \frac{1}{(4 \pi t)^{\frac{d+1}{2}}} e^{-\frac{1}{4 t}\left[\left(x_{I}^{\prime}-x_{I}\right)\left(x^{\prime I}-x^{I}\right)+\left(y^{\prime \alpha}-y^{\alpha}+m^{\alpha}\right) g_{\alpha \beta}\left(y^{\prime \beta}-y^{\beta}+m^{\beta}\right)\right]},}
$$


with trace

$$
\operatorname{Tr} e^{-t \hat{\mathrm{H}}_{\mathbb{R}}{ } \times \mathbb{T}^{q+1}}=\frac{V_{d-q} \mathrm{~V}_{q+1}}{(4 \pi t)^{\frac{d+1}{2}}} \sum_{m^{\alpha} \in \mathbb{Z}^{q+1}} e^{-\frac{1}{4 t} m^{\alpha} g_{\alpha \beta} m^{\beta}}
$$

while the partition function, after changing the integration variable $t^{\prime}=\frac{1}{4 \pi t}\left(\mathrm{~V}_{q+1}\right)^{\frac{2}{q+1}}$, is given by

$$
\ln Z_{d, q}(g)=\frac{V_{d-q}}{2\left(\mathrm{~V}_{q+1}\right)^{\frac{d-q}{q+1}}} \int_{0}^{\infty} \mathrm{d} t t^{\frac{d+1}{2}-1}\left(\theta_{q+1}(t \hat{g})-1\right) .
$$

When comparing this expression with equation (4.22), it is consistent with the suggestion that the completed $\mathrm{SL}(n, \mathbb{Z})$ Eisenstein series is related to the Mellin transform of the Riemann theta function $\theta_{n}(t \hat{g})$ minus 1 ,

$$
\xi(z, n ; \hat{g})=\frac{1}{2} \int_{0}^{\infty} \mathrm{d} t t^{\frac{z}{2}-1}\left(\theta_{n}(t \hat{g})-1\right), \quad \mathfrak{R e}(z)>1
$$

in the sense that the equality of (4.22) and (4.122) corresponds to the case $z=d+1$, $n=q+1$.

From the world-line approach, the Fourier transform and the high temperature/large box expansion may also be obtained directly without necessarily passing through canonical quantization by the following generalization of the approach of [1], section 2.4. The argument of the exponential, $-\pi t n_{\alpha} \hat{g}^{\alpha \beta} n_{\beta}$ in (4.118), when using the dual ADM parametrization in (4.82) together with $N_{D}^{\iota}=\hat{\theta}_{i}^{D \iota} b_{D} \mu_{D}^{i}$, becomes

$$
\begin{aligned}
-\pi t b_{D}^{-\frac{2}{q+1}}\left[n_{\iota} \hat{h}_{D}^{\iota \kappa} n_{\kappa}+\right. & \left.2 n_{\iota} N_{D}^{\iota} n_{d+1}+\left(b_{D}^{2}+N_{D}^{\iota} N_{\iota}^{D}\right) n_{d+1}^{2}\right] \\
& =-\pi t b_{D}^{-\frac{2}{q+1}}\left[\left(n_{\iota}+N_{\iota}^{D} n_{d+1}\right) \hat{h}_{D}^{\iota \kappa}\left(n_{\kappa}+N_{\kappa}^{D} n_{d+1}\right)+b_{D}^{2} n_{d+1}^{2}\right] .
\end{aligned}
$$

One then splits the sum over $n_{\alpha} \in \mathbb{Z}^{q+1} /(0, \ldots, 0)$. The first part is given by $n_{d+1}=0$. After the change of variables $t^{\prime}=\left(b_{D}\right)^{-\frac{2}{q+1}} t$ and using $\mathrm{V}_{q+1}=\left(\mathrm{V}_{q}\right)^{\frac{q+1}{q}} b$, the result is

$$
\frac{V_{d-q}}{\left(\mathrm{~V}_{q}\right)^{\frac{d-q}{q}}} \frac{1}{\left(b b_{D}\right)^{\frac{d-q}{q+1}}} \xi\left(q-d, q ; \hat{g}_{D}^{-1}\right) .
$$

After using the reflection formula (4.21) and also the first of (4.83), this reduces to the second term of (4.85).

For the second part, since it involves $n_{d+1} \neq 0$, one may use a Poisson resummation for the free sum over $n_{\iota} \in \mathbb{Z}^{q}$ to get

$$
\begin{aligned}
& \frac{V_{d-q}}{2\left(\mathrm{~V}_{q}\right)^{\frac{d-q}{q}}} \frac{\left(b_{D}\right)^{\frac{q}{q+1}}}{(b)^{\frac{d-q}{q+1}}} \int_{0}^{\infty} \mathrm{d} t t^{-\frac{d}{2}-1} \sum_{n_{d+1} \in \mathbb{Z}}^{\prime} \sum_{m^{\iota} \in \mathbb{Z}^{q}} \\
& \quad e^{-\pi t^{-1} b_{D}^{\frac{2}{q+1}}\left[\left(m^{\iota}-i t b_{D}^{-\frac{2}{q+1}} N_{D}^{\iota} n_{d+1}\right) \hat{h}_{\iota \kappa}^{D}\left(m^{\kappa}-i t b_{D}^{-\frac{2}{q+1}} N_{D}^{\kappa} n_{d+1}\right)\right]-\pi t b_{D}^{-\frac{2}{q+1}}\left(b_{D}^{2}+N_{\iota}^{D} N_{D}^{\iota}\right) n_{d+1}^{2} .}
\end{aligned}
$$


If all $m^{\iota}$ vanish, this reduces to

$$
\frac{V_{d-q}}{2\left(\mathrm{~V}_{q}\right)^{\frac{d-q}{q}}} \frac{\left(b_{D}\right)^{\frac{q}{q+1}}}{(b)^{\frac{d-q}{q+1}}} \int_{0}^{\infty} \mathrm{d} t t^{-\frac{d}{2}-1} \sum_{n_{d+1} \in \mathbb{Z}}^{\prime} e^{-\pi t b_{D}^{\frac{2 q}{q+1}} n_{d+1}^{2}}=\frac{V_{d-q}}{\left(\mathrm{~V}_{q}\right)^{\frac{d-q}{q}}}\left(1+\mu_{i} \mu^{i}\right)^{\frac{d-q}{2 q}} b_{D}^{d} \xi(-d),
$$

where the change of integration variables $t^{\prime}=t\left(b_{D}\right)^{\frac{2 q}{q+1}}$ has been used. After using the reflection formula (2.42), this agrees with the "corrected" black body result, that is to say first term of (4.85) or of (4.86).

The remaining sum is

$$
\begin{aligned}
\frac{V_{d-q}}{2\left(\mathrm{~V}_{q}\right)^{\frac{d-q}{q}}}\left(1+\mu_{1} \mu^{i}\right)^{\frac{d-q}{2 q}} b_{D}^{\frac{d}{q+1}} \sum_{n_{d+1} \in \mathbb{Z} m^{\iota} \in \mathbb{Z}^{q}}^{\prime} & \sum^{\prime} \\
& \int_{0}^{\infty} d t t^{-\frac{d}{2}-1} e^{-\pi t^{-1} b_{D}^{\frac{2}{q+1}} m^{\iota} \hat{h}_{\iota \kappa}^{D} m^{\kappa}-\pi t\left(b_{D}\right)^{\frac{2 q}{q+1}} n_{d+1}^{2}+2 \pi i m^{\iota} N_{\iota}^{D} n_{d+1} .}
\end{aligned}
$$

When using (8.432.6) of [49],

$$
\int_{0}^{\infty} \mathrm{d} t t^{-\nu-1} e^{-t-\frac{z^{2}}{4 t}}=2\left(\frac{z}{2}\right)^{-\nu} K_{\nu}(z)
$$

it follows that

$$
\begin{aligned}
\int_{0}^{\infty} \mathrm{d} t t^{-\frac{d}{2}-1} e^{-\pi t^{-1}} b_{D}^{\frac{2}{q+1}} m^{\iota(q)} \hat{g}_{\iota \kappa}^{D} m^{\kappa}-\pi t b_{D}^{\frac{2 q}{q+1}} n_{d+1}^{2} \\
=2\left(b_{D}\right)^{\frac{d(q-1)}{2(q+1)}}\left(\frac{\sqrt{m^{\iota} \hat{h}_{\iota \kappa}^{D} m^{\kappa}}}{n_{d+1}}\right)^{-\frac{d}{2}} K_{\frac{d}{2}}\left(2 \pi n_{d+1} b_{D} \sqrt{m^{\iota} \hat{h}_{\iota \kappa}^{D} m^{\kappa}}\right),
\end{aligned}
$$

the remaining sum agrees with the last line of (4.85).

\subsection{Partition function on $\mathbb{T}^{d+1}$ and harmonic anomaly}

The partition function on $\mathbb{T}^{d+1}$, computed as in (4.22), is given by the divergent expression

$$
\ln Z_{d, d}^{\text {div }}(g)=\xi(d+1, d+1 ; \hat{g}) .
$$

It may be regularized by studying the behavior of $\xi(\epsilon+d+1, d+1 ; \hat{g})$ in the limit $\epsilon \rightarrow 0$,

$$
\ln Z_{d+\epsilon, d}^{\mathrm{reg}}(g)=\xi(d+1+\epsilon, d+1 ; \hat{g}) .
$$

According to (4.70), its Fourier expansion is given by

$$
\begin{aligned}
\ln Z_{d+\epsilon, d}^{\mathrm{reg}}\left(g^{\mathrm{ADM}}\right)= & b^{1+\frac{\epsilon}{d+1}} \xi(d+1+\epsilon, d ; \hat{h})+b^{-\frac{\epsilon d}{d+1}} \xi(1+\epsilon)+2 b^{\frac{2 \epsilon-(\epsilon-1)(d+1)}{2(d+1)}} \\
& \sum_{n_{\iota} \in \mathbb{Z}^{d}}^{\prime} \sum_{l \in \mathbb{N}^{*}}\left(\frac{\sqrt{n_{\iota} \hat{h}^{\iota \kappa} n_{\kappa}}}{l}\right)^{\frac{\epsilon+1}{2}} K_{\frac{\epsilon+1}{2}}\left(2 \pi l b \sqrt{n_{\iota} \hat{h}^{\iota \kappa} n_{\kappa}}\right) e^{2 \pi i l n_{\iota} N^{\iota}} .
\end{aligned}
$$


For $\epsilon \rightarrow 0$, the first and last terms are finite and given respectively by $b \xi(d+1, d ; \hat{h})$ and, when using that $K_{\frac{1}{2}}(x)=\sqrt{\frac{\pi}{2 x}} e^{-x}$,

$$
\sum_{n_{\iota} \in \mathbb{Z}^{d}}^{\prime} \sum_{l \in \mathbb{N}^{*}} \frac{e^{2 \pi i l\left(n_{\iota} N^{\iota}+i b \sqrt{n_{\iota} \hat{h}^{\iota \kappa} n_{\kappa}}\right)}}{l}=-\sum_{n_{\iota} \in \mathbb{Z}^{d}}^{\prime} \ln \left(1-e^{2 \pi i\left(n_{\iota} N^{\iota}+i b \sqrt{n_{\iota} \hat{h}^{\iota \kappa} n_{\kappa}}\right)}\right) .
$$

The second term is divergent for $\epsilon \rightarrow 0$. When using (3.107), it is explicitly given by

$$
\begin{aligned}
b^{-\frac{\epsilon d}{d+1}} \xi(\epsilon+1) & =\frac{\Gamma\left(\frac{\epsilon+1}{2}\right)}{\pi^{\frac{\epsilon+1}{2}}} b^{-\frac{\epsilon d}{d+1}} \zeta(\epsilon+1) \\
& =\frac{1}{\epsilon}+\gamma+\frac{1}{2}\left(\frac{\Gamma^{\prime}\left(\frac{1}{2}\right)}{\Gamma\left(\frac{1}{2}\right)}-\sqrt{\pi} \ln \pi\right)-\frac{d}{d+1} \ln b+O(\epsilon)
\end{aligned}
$$

Putting the terms back together, and using $\frac{\Gamma^{\prime}\left(\frac{1}{2}\right)}{\Gamma\left(\frac{1}{2}\right)}=-2 \ln 2-\gamma$, it follows that

$$
\begin{aligned}
\ln Z_{d+\epsilon, d}^{\mathrm{reg}}\left(g^{\mathrm{ADM}}\right)= & b \xi(d+1, d ; \hat{h})-\sum_{n_{\iota} \in \mathbb{Z}^{d}}^{\prime} \ln \left(1-e^{2 \pi i\left(n_{\iota} N^{\iota}+i b \sqrt{n_{\iota} \hat{h}^{\iota \kappa} n_{\kappa}}\right)}\right) \\
& +\frac{1}{\epsilon}+\frac{\gamma-\sqrt{\pi} \ln \pi}{2}-\ln 2-\frac{d}{d+1} \ln b+O(\epsilon) .
\end{aligned}
$$

The renormalized partition function is then defined by subtracting the pole, together with irrelevant purely numerical factors, and then taking the limit,

$$
\begin{aligned}
\ln Z_{d, d}^{\mathrm{ren}}\left(g^{\mathrm{ADM}}\right) & =\lim _{\epsilon \rightarrow 0}\left[\ln Z_{d+\epsilon, d}^{\mathrm{reg}}\left(g^{\mathrm{ADM}}\right)-\left(\frac{1}{\epsilon}+\frac{\gamma-\sqrt{\pi} \ln \pi}{2}-\ln 2\right)\right] \\
& =b \xi(d+1, d ; \hat{h})-\sum_{n_{\iota} \in \mathbb{Z}^{d}}^{\prime} \ln \left(1-e^{2 \pi i\left(n_{\iota} N^{\iota}+i b \sqrt{n_{\iota} \hat{h}^{\iota \kappa} n_{\kappa}}\right)}\right)-\frac{d}{d+1} \ln b .
\end{aligned}
$$

\section{Remarks}

(i) The first term contains the Casimir energy for $\mathbb{T}^{d}$. Together with the second, their exponential is what replaces the expression in terms of the Dedekind $\eta$ function and its complex conjugate in (3.100). The last term is a non-trivial contributions due to the pole.

(ii) As in [1], Proposition 2, one notes that the renormalized result continues to be $\mathrm{SL}(d+1, \mathbb{Z})$ invariant since the residue of the pole and the numerical factors do not depend on the parameters of the torus.

(iii) When applying (A.18) to the formal divergent expression $\ln Z_{d, d}^{\text {div }}(g)$ in (4.131), it would follow naively that $\Delta_{\mathrm{SL}(d+1)} \ln Z_{d, d}^{\text {div }}=0$. However, for the regularized partition function one finds,

$$
\Delta_{\mathrm{SL}(d+1)} \ln Z_{d+\epsilon, d}^{\mathrm{reg}}\left(g^{\mathrm{ADM}}\right)=\frac{\epsilon(d+1+\epsilon) d}{4(d+1)} \ln Z_{d+\epsilon, d}^{\mathrm{reg}}\left(g^{\mathrm{ADM}}\right) .
$$


Using now that $\Delta_{\mathrm{SL}(d+1)}$ does not depend on $\epsilon$, while the subtracted terms do not involve parameters of $g_{\alpha \beta}^{\mathrm{ADM}}$, the definition of the renormalized partition function in terms of the regularized one in the first line of (4.137), then yields the harmonic anomaly [1],

$$
\Delta_{\mathrm{SL}(d+1)} \ln Z_{d, d}^{\mathrm{ren}}\left(g^{\mathrm{ADM}}\right)=\frac{d}{4} .
$$

As a consequence, one now finds

$$
(d+1) t^{d-1} \hat{J}^{\alpha \beta \gamma \delta}\left\langle\Delta \hat{\mathcal{D}}_{\alpha \beta} \Delta \hat{\mathcal{D}}_{\gamma \delta}\right\rangle-d(d+3) t^{\frac{d-1}{2}} \hat{g}^{\alpha \beta}\left\langle\hat{\mathcal{D}}_{\alpha \beta}\right\rangle=d(d+1),
$$

rather than the naive result of 0 on the right hand side that one would get by extrapolating (4.37) to $q=d$.

When directly applying the ADM form of the Laplacian in equation (A.26) to the renormalized result, it follows that the harmonic anomaly comes purely from the $-\frac{d}{d+1} \ln b$ term, and as a consequence,

$$
\Delta_{\mathrm{SL}(d+1), \mathrm{ADM}}\left[b \xi(d+1, d ; \hat{h})-\sum_{n_{\iota} \in \mathbb{Z}^{d}}^{\prime} \ln \left(1-e^{2 \pi i\left(n_{\iota} N^{\iota}+i b \sqrt{n_{\iota} \hat{h}^{\iota \kappa} n_{\kappa}}\right)}\right)\right]=0 .
$$

\section{Outlook}

For a free massless boson on flat backgrounds, we have obtained exact analytic results for the partition function in terms of well-studied modular forms. High respectively low temperature expansions can be re-summed to all orders and various conjectures about modular invariance in higher dimensions can be explicitly checked. The detailed understanding that has been achieved should prove useful in more non-trivial situations where approximation methods adapted to either the high or low temperature regime have to be applied.

From the viewpoint of boundary conditions, the most symmetric approach consists in taking the field periodic in all spacetimes dimensions, or more generally, to compute the partition function on $\mathbb{T}^{d+1}$. The detailed analysis shows however that the presence of large spatial dimensions acts as a regulator so that certain divergences and zero mode issues do not show up in that case.

It would be interesting to generalize the computation of free field partition functions here to that of thermal correlators in interacting theories (see e.g. [50, 51] for recent work in this context) and to understand what survives of the present considerations in the case of non-flat backgrounds.

From a mathematical perspective, relations (2.71), (3.64), (3.92), (4.123) (4.70) between zeta functions or Eisenstein series and their Fourier series or (inverse) Mellin transforms that follow from the equivalence of various quantization methods hold for integer $z=d+1$ or half integer variables $s=\frac{d+1}{2}$. In order to access more general complex values of $z$ or $s$, one may think about the computation of these partition functions in the framework of dimensional regularization, as discussed in the last section. 
More obvious generalizations include the study of the massive case as well as that of fermions. Finally, it would also be interesting to understand from first principles if there is a reason why the same Eisenstein series occur in string theory and in these scalar field partition functions and why the partition function for a massless scalar field on $R^{d-q} \times T^{q+1}$ should be an eigenfunction of the $S L(q+1)$ Laplacian.

\section{Acknowledgments}

The authors are grateful to A. Kleinschmidt for helpful suggestions and to T. Petkou and A. Aggarwal for useful discussions. This work is supported by the F.R.S.-FNRS Belgium through conventions FRFC PDR T.1025.14 and IISN 4.4503.15, as well as a research fellowship for M. Bonte. The work of F. Alessio has been partially supported by a fellowship from the "Fondazione Angelo Della Riccia". G. B. is grateful to the Erwin Schrödinger International Institute for Mathematics and Physics for hospitality during the final stages of this work.

\section{A $\operatorname{GL}(n, \mathbb{R}) / \operatorname{SO}(n, \mathbb{R})$ and $\operatorname{SL}(n, \mathbb{R}) / \operatorname{SO}(n, \mathbb{R})$ Laplacians}

In this appendix, we provide some background on the Laplacians that are used in section 4.3.

One starts from the (generalized) DeWitt supermetric [52] (see also e.g. [53, 54])

$$
G^{\alpha \beta \gamma \delta}(\eta, \lambda ; n)=\frac{g^{\frac{\eta}{2}}}{2}\left(g^{\alpha \gamma} g^{\beta \delta}+g^{\alpha \delta} g^{\beta \gamma}-2 \lambda g^{\alpha \beta} g^{\gamma \delta}\right),
$$

with $\lambda=1, \eta=1, n=3$ in [52] and $\lambda=0=\eta$ in [1]. The inverse supermetric exists when $\lambda \neq \frac{1}{n}$ and is given by

$$
\begin{aligned}
G_{\alpha \beta \gamma \delta}(\eta, \lambda ; n) & =\frac{g^{\frac{-\eta}{2}}}{2}\left(g_{\alpha \gamma} g_{\beta \delta}+g_{\alpha \delta} g_{\beta \gamma}-\frac{2 \lambda}{\lambda n-1} g_{\alpha \beta} g_{\gamma \delta}\right), \\
G_{\alpha \beta \mu \nu}(\eta, \lambda ; n) G^{\mu \nu \gamma \delta}(\eta, \lambda ; n) & =\frac{1}{2}\left(\delta_{\alpha}^{\gamma} \delta_{\beta}^{\delta}+\delta_{\alpha}^{\delta} \delta_{\beta}^{\gamma}\right) .
\end{aligned}
$$

For the determinant $G(\eta, \lambda ; n)=\operatorname{det} G^{\alpha \beta \gamma \delta}(\eta, \lambda ; n)$, one uses that

$$
-\delta \ln G(\eta, \lambda ; n)=G^{\alpha \beta \gamma \delta}(\eta, \lambda ; n) \delta G_{\alpha \beta \gamma \delta}(\eta, \lambda ; n)=(n+1)\left(1-\frac{n \eta}{4}\right) \delta \ln g,
$$

to infer that

$$
G(\eta, \lambda ; n)=a g^{-(n+1)\left(1-\frac{n \eta}{4}\right)},
$$

for some constant $a$. One then finds $G(1,1 ; 3)=a g^{-1}$ as in $[52]$ and $G(0,0 ; n)=a g^{-(n+1)}$ for the case relevant to [1] and to us here. For simplicity, we now denote $G^{\alpha \beta \gamma \delta}(0,0 ; n)=G^{\alpha \beta \gamma \delta}$ in the following.

According to [1], the metric on $\mathrm{GL}(n, \mathbb{R}) / \mathrm{SO}(n, \mathbb{R})$ is given by the supermetric

$$
d s_{\mathrm{GL}(n)}^{2}=G^{\alpha \beta \gamma \delta} \mathrm{d} g_{\alpha \beta} \mathrm{d} g_{\gamma \delta}, \quad G^{\alpha \beta \gamma \delta}=\frac{1}{2}\left(g^{\alpha \gamma} g^{\beta \delta}+g^{\alpha \delta} g^{\beta \gamma}\right) .
$$


The inverse is $G_{\alpha \beta \gamma \delta}$, with indices on the supermetric lowered and raised with $g_{\alpha \beta}$ and its inverse. In particular,

$$
G_{\alpha \beta}^{\gamma \delta}=\delta_{\alpha \beta}^{\gamma \delta}=\frac{1}{2}\left(\delta_{\alpha}^{\gamma} \delta_{\beta}^{\delta}+\delta_{\beta}^{\gamma} \delta_{\alpha}^{\delta}\right)
$$

Since the determinant of the supermetric is $G=a g^{-(n+1)}$, the associated Laplacian is

$$
\Delta_{\mathrm{GL}(n)}=G^{-\frac{1}{2}} \frac{\partial}{\partial g_{\alpha \beta}}\left(G^{\frac{1}{2}} G_{\alpha \beta \gamma \delta}\right) \frac{\partial}{\partial g_{\gamma \delta}}, \quad \frac{\partial g_{\alpha \beta}}{\partial g_{\gamma \delta}}=\delta_{\alpha \beta}^{\gamma \delta},
$$

or explicitly,

$$
\Delta_{\mathrm{GL}(n)}=G_{\alpha \beta \gamma \delta} \frac{\partial^{2}}{\partial g_{\alpha \beta} \partial g_{\gamma \delta}}+\frac{n+1}{2} g_{\alpha \beta} \frac{\partial}{\partial g_{\alpha \beta}} .
$$

When applied to the Eisenstein series, one finds

$$
\begin{aligned}
\Delta_{\mathrm{GL}(n)} f_{s}(n ; g) & =\frac{s(2 s-n+1)}{2} f_{s}(n ; g), \\
\Delta_{\mathrm{GL}(n)} \xi(z, n ; g) & =\frac{z(z-n+1)}{4} \xi(z, n ; g) .
\end{aligned}
$$

Consider now the change of variables from $g_{\alpha \beta}$ to $t, \hat{g}_{\alpha \beta}$,

$$
g_{\alpha \beta}=t \hat{g}_{\alpha \beta}
$$

with $t=g^{\frac{1}{n}}, \hat{g}=1$. It follows that

$$
\begin{aligned}
& \frac{\partial t}{\partial g_{\alpha \beta}}=\frac{1}{n} \operatorname{tg}^{\alpha \beta}, \quad \frac{\partial g_{\alpha \beta}}{\partial t}=\hat{g}_{\alpha \beta}, \quad g_{\alpha \beta} \frac{\partial \hat{g}_{\gamma \delta}}{\partial g_{\alpha \beta}}=0=g^{\alpha \beta} \frac{\partial g_{\alpha \beta}}{\partial \hat{g}_{\gamma \delta}}, \\
& \frac{\partial \hat{g}_{\alpha \beta}}{\partial g_{\gamma \delta}}=\frac{1}{t} J_{\alpha \beta}^{\gamma \delta}, \quad \frac{\partial g_{\alpha \beta}}{\partial \hat{g}_{\gamma \delta}}=t J_{\alpha \beta}^{\gamma \delta}, \quad J_{\alpha \beta}^{\gamma \delta}=\frac{1}{2}\left(\delta_{\alpha}^{\gamma} \delta_{\beta}^{\delta}+\delta_{\alpha}^{\delta} \delta_{\beta}^{\gamma}-\frac{2}{n} g^{\gamma \delta} g_{\alpha \beta}\right) .
\end{aligned}
$$

with

$$
\begin{aligned}
J_{\alpha \beta}^{\gamma \delta} J_{\gamma \delta}{ }^{\rho \sigma} & =J_{\alpha \beta}{ }^{\rho \sigma}, & g^{\alpha \beta} J_{\alpha \beta}^{\gamma \delta} & =0=J_{\alpha \beta}^{\gamma \delta} g_{\gamma \delta}, \\
J_{\alpha \beta}{ }^{\alpha \beta} & =\frac{n(n+1)-2}{2}, & J_{\alpha}{ }^{\beta \gamma \alpha} & =\frac{n(n+1)-2}{2 n} g^{\beta \gamma} .
\end{aligned}
$$

Let $\hat{G}^{\alpha \beta \gamma \delta}, \hat{J}_{\alpha \beta}^{\gamma \delta}$ be the same expressions as $\hat{G}^{\alpha \beta \gamma \delta}, \hat{J}_{\alpha \beta}{ }^{\gamma \delta}$ with $g_{\alpha \beta}$ and its inverse replaced by $\hat{g}_{\alpha \beta}$ and its inverse, including for raising and lowering indices. In particular $\hat{J}_{\alpha \beta}{ }^{\gamma \delta}=J_{\alpha \beta}{ }^{\gamma \delta}$. In the new parametrization, the supermetric becomes

$$
d s_{\mathrm{GL}(n)}^{2}=\hat{g}^{\alpha \gamma} \hat{g}^{\beta \delta} \mathrm{d} \hat{g}_{\alpha \beta} \mathrm{d} \hat{g}_{\gamma \delta}+n \mathrm{~d}(\ln t)^{2}=\hat{J}^{\alpha \beta \gamma \delta} \mathrm{d} \hat{g}_{\alpha \beta} \mathrm{d} \hat{g}_{\gamma \delta}+n(\mathrm{~d} \ln t)^{2},
$$

so that the metric on $\mathrm{SL}(n, \mathbb{R}) / \mathrm{SO}(n, \mathbb{R})$ is given by

$$
d s_{\mathrm{SL}(n)}^{2}=\hat{J}^{\alpha \beta \gamma \delta} \mathrm{d} \hat{g}_{\alpha \beta} \mathrm{d} \hat{g}_{\gamma \delta}=J^{\alpha \beta \gamma \delta} \mathrm{d} g_{\alpha \beta} \mathrm{d} g_{\gamma \delta} .
$$

It follows from (A.14) that the associated Laplacian satisfies

$$
\Delta_{\mathrm{GL}(n)}=\Delta_{\mathrm{SL}(n)}+\frac{1}{n}\left(t \frac{\partial}{\partial t}\right)^{2}
$$


and is thus explicitly given by

$$
\begin{aligned}
\Delta_{\mathrm{SL}(n)} & =J_{\alpha \beta \gamma \delta} \frac{\partial^{2}}{\partial g_{\alpha \beta} \partial g_{\gamma \delta}}+\frac{n(n+1)-2}{2 n} g_{\alpha \beta} \frac{\partial}{\partial g_{\alpha \beta}} \\
& =\hat{J}_{\alpha \beta \gamma \delta} \frac{\partial^{2}}{\partial \hat{g}_{\alpha \beta} \partial \hat{g}_{\gamma \delta}} .
\end{aligned}
$$

It follows that

$$
\begin{gathered}
\Delta_{\mathrm{SL}(n)} f_{s}(n ; \hat{g})=\frac{s(n-1)(2 s-n)}{2 n} f_{s}(n ; \hat{g}), \\
\Delta_{\mathrm{SL}(n)} \xi(z, n ; \hat{g})=\frac{z(n-1)(z-n)}{4 n} \xi(z, n ; \hat{g}) .
\end{gathered}
$$

In particular, setting $n=2$, yields $\Delta_{\mathrm{SL}(2)} f_{s}(2 ; \hat{g})=\frac{s(s-1)}{2} f_{s}(2 ; \hat{g})$. Since

$$
\begin{aligned}
\mathrm{d} s_{\mathrm{SL}(2)}^{2}=\left(\hat{g}^{11}\right)^{2}\left(\mathrm{~d} \hat{g}_{11}\right)^{2}+4 \hat{g}^{11} \hat{g}^{12} \mathrm{~d} \hat{g}_{11} \mathrm{~d} \hat{g}_{12}+2\left(\hat{g}^{12}\right)^{2} \mathrm{~d} \hat{g}_{11} \mathrm{~d} \hat{g}_{22} \\
+2\left[\hat{g}^{11} \hat{g}^{22}+\left(\hat{g}^{12}\right)^{2}\right]\left(\mathrm{d} \hat{g}_{12}\right)^{2}+4 \hat{g}^{12} \hat{g}^{22} \mathrm{~d} \hat{g}_{12} \mathrm{~d} \hat{g}_{22}+\left(\hat{g}^{22}\right)^{2}\left(\mathrm{~d} \hat{g}_{22}\right)^{2},
\end{aligned}
$$

reduces to

$$
\mathrm{d} s_{\mathrm{SL}(2)}^{2}=\frac{2}{\tau_{2}^{2}}\left(\mathrm{~d} \tau_{1}^{2}+\mathrm{d} \tau_{2}^{2}\right)
$$

when using the parametrization in the last two lines of (4.30), it follows that

$$
\Delta_{\mathrm{SL}(2)}=\frac{\tau_{2}^{2}}{2}\left(\partial_{\tau_{1}}^{2}+\partial_{\tau_{2}}^{2}\right)=\frac{1}{2} \Delta_{\mathbb{H}}
$$

In order to work out the supermetric and the Laplacian in ADM parametrization, we start with the decomposition

$$
\begin{aligned}
& g^{\alpha \gamma} g^{\beta \delta} \mathrm{d} g_{\alpha \beta} \mathrm{d} g_{\gamma \delta}=g^{\iota \xi} g^{\kappa \lambda} \mathrm{d} g_{\iota \kappa} \mathrm{d} g_{\xi \lambda}+4 g^{\iota \xi} g^{\kappa, q+1} \mathrm{~d} g_{\iota \kappa} d g_{\xi, q+1} \\
& \quad+2 g^{\iota, q+1} g^{\kappa, q+1} \mathrm{~d} g_{\iota \kappa} \mathrm{d} g_{q+1, q+1}+2\left(g^{\iota \kappa} g^{q+1, q+1}+g^{\iota, q+1} g^{\kappa, q+1}\right) \mathrm{d} g_{\iota, q+1} \mathrm{~d} g_{\kappa, q+1} \\
& \quad+4 g^{\iota, q+1} g^{q+1, q+1} \mathrm{~d} g_{\iota, d+1} \mathrm{~d} g_{q+1, q+1}+\left(g^{q+1, q+1}\right)^{2}\left(\mathrm{~d} g_{q+1, q+1}\right)^{2} .
\end{aligned}
$$

In terms of the ADM parametrization (4.39), the supermetric becomes

$$
\begin{aligned}
\mathrm{d} s_{\mathrm{GL}(q+1), \mathrm{ADM}}^{2} & =G_{\mathrm{ADM}}^{\alpha \beta \gamma \delta} \mathrm{d} g_{\alpha \beta}^{\mathrm{ADM}} \mathrm{d} g_{\gamma \delta}^{\mathrm{ADM}} \\
& ={ }^{(q)} G^{\iota \kappa \lambda \xi} \mathrm{d} h_{\iota \kappa} \mathrm{d} h_{\lambda \xi}+\frac{2}{N^{2}} h_{\iota \kappa} \mathrm{d} N^{\iota} d N^{\kappa}+4(\mathrm{~d} \ln N)^{2} .
\end{aligned}
$$

Since $g_{\alpha \beta}^{\mathrm{ADM}}=(N \sqrt{h})^{2} \hat{g}_{\alpha \beta}^{\mathrm{ADM}}$, it follows from (A.14) that

$$
\begin{aligned}
\mathrm{d} s_{\mathrm{SL}(q+1), \mathrm{ADM}}^{2}= & \hat{G}_{\mathrm{ADM}}^{\alpha \beta \gamma \delta} \mathrm{d} \hat{g}_{\alpha \beta}^{\mathrm{ADM}} \mathrm{d} \hat{g}_{\gamma \delta}^{\mathrm{ADM}}={ }^{(q)} G^{\iota \kappa \lambda \xi} \mathrm{d} h_{\iota \kappa} \mathrm{d} h_{\lambda \xi} \\
& +\frac{2}{N^{2}} h_{\iota \kappa} \mathrm{d} N^{\iota} \mathrm{d} N^{\kappa}+4(\mathrm{~d} \ln N)^{2}-\frac{4}{q+1}\left(\mathrm{~d} \ln N+\frac{1}{2} \mathrm{~d} \ln h\right)^{2} .
\end{aligned}
$$

Since $h_{\iota \kappa}=(h)^{\frac{1}{q}} \hat{h}_{\iota \kappa}$ it follows by using again (A.14) that

$$
\begin{aligned}
& \mathrm{d} s_{\mathrm{SL}(q+1), \mathrm{ADM}}^{2}={ }^{(q)} \hat{G}^{\iota \kappa \lambda \xi} \mathrm{d} \hat{h}_{\iota \kappa} \mathrm{d} \hat{h}_{\lambda \xi} \\
& \quad+\frac{2}{N^{2}} h_{\iota \kappa} \mathrm{d} N^{\iota} \mathrm{d} N^{\kappa}+4(\mathrm{~d} \ln N)^{2}-\frac{4}{q+1}\left(\mathrm{~d} \ln N+\frac{1}{2} \mathrm{~d} \ln h\right)^{2}+\frac{1}{q}(\mathrm{~d} \ln h)^{2} .
\end{aligned}
$$


Changing variables to $b, \mathrm{~V}_{q}$ with $N=b\left(\mathrm{~V}_{q}\right)^{\frac{1}{q}}, h=\left(\mathrm{V}_{q}\right)^{2}$, gives

$$
d s_{\mathrm{SL}(q+1), \mathrm{ADM}}^{2}={ }^{(q)} \hat{G}^{\iota \kappa \lambda \xi} \mathrm{d} \hat{h}_{\iota \kappa} \mathrm{d} \hat{h}_{\lambda \xi}+\frac{2}{b^{2}} \hat{h}_{\iota \kappa} \mathrm{d} N^{\iota} d N^{\kappa}+\frac{4 q}{q+1}(\mathrm{~d} \ln b)^{2} .
$$

The determinant is given by

$$
\hat{G}_{\mathrm{ADM}}={ }^{(q)} \hat{G} \frac{2^{q+2}}{b^{2(q+1)}} \frac{4 q}{q+1},
$$

where ${ }^{(q)} \hat{G}$ does not depend on either $b$ or $N^{\iota}$. The associated Laplacian is then given by

$$
\Delta_{\mathrm{SL}(q+1), \mathrm{ADM}}=\Delta_{\mathrm{SL}(q)}+\frac{b^{2} \hat{h}^{\iota \kappa}}{2} \frac{\partial}{\partial N^{\iota}} \frac{\partial}{\partial N^{\kappa}}+\frac{q+1}{4 q}\left[b^{2} \frac{\partial^{2}}{\partial b^{2}}-(q-1) b \frac{\partial}{\partial b}\right] .
$$

When taking into account that $\Delta_{\mathrm{SL}(1), \mathrm{ADM}}$ vanishes and identifying $b=\tau_{2}, N^{1}=\tau_{1}$, this formula correctly reduces to the $\mathrm{SL}(2, \mathbb{Z})$ Laplacian (A.21). It may be used recursively to construct an explicit expression of the $\mathrm{SL}(q+1, \mathbb{Z})$ Laplacian by using a similar parametrization of $\hat{h}_{\iota \kappa}$ in terms of variables $b_{(q)}, N_{(q)}^{\iota d-1}$.

\section{B Trigonometric expansions}

In this appendix, we derive expansions for the Epstein zeta function and the Eisenstein series in terms of trigonometric functions.

The Epstein zeta function is defined as

$$
\zeta\left(s ; a_{1}, \ldots, a_{p}\right)=\sum_{n_{1}, \ldots, n_{p} \in \mathbb{Z}^{p}}^{\prime} \frac{1}{\left(a_{1} n_{1}^{2}+\ldots+a_{p} n_{p}^{2}\right)^{s}},
$$

and satisfies the functional relation

$$
\Gamma(s) \zeta\left(s ; a_{1}, \ldots, a_{p}\right)=\frac{\pi^{2 s-\frac{p}{2}}}{\sqrt{a_{1} \ldots a_{p}}} \Gamma\left(\frac{p}{2}-s\right) \zeta\left(\frac{p}{2}-s ; \frac{1}{a_{1}}, \ldots, \frac{1}{a_{p}}\right) .
$$

The real analytic Eisenstein series $f_{s}(\tau, \bar{\tau})$ and the Epstein zeta function are related by

$$
f_{s}\left(i \tau_{2},-i \tau_{2}\right)=\sum_{(n, m) \in \mathbb{Z}^{2}}^{\prime} \frac{\tau_{2}^{s}}{\left(n^{2} \tau_{2}^{2}+m^{2}\right)^{s}}=\tau_{2}^{s} \zeta\left(s ; \tau_{2}^{2}, 1\right) .
$$

The Fourier transform of the real analytic Eisenstein series in (3.64), in the particular case of purely imaginary $\tau$, may be directly obtained from a Sommerfeld-Watson transform, which we briefly review first. Consider the series

$$
S=\sum_{m \in \mathbb{Z}} f(m)
$$

and the complex function

$$
F(z)=\pi f(z) \cot (\pi z)
$$


having simple poles in $z=m, m \in \mathbb{Z}$, with residues

$$
\left.\operatorname{Res}[F(z)]\right|_{z=m}=f(m) .
$$

Assuming that

$$
f(z) \stackrel{|z| \rightarrow \infty}{\longrightarrow} \frac{1}{|z|^{1+\epsilon}}, \quad \epsilon>0,
$$

the integral along a circle $C_{R}$ of radius $R$ centred at the origin vanishes as $R \rightarrow \infty$

$$
0=\oint_{C_{\infty}} F(z) d z=2 \pi i\left[\left.\sum_{m \in \mathbb{Z}} \operatorname{Res}[F(z)]\right|_{z=m}+\left.\sum_{i} \operatorname{Res}[F(z)]\right|_{z=z_{i}}\right],
$$

where we used the residue theorem and where $z_{i}$ are the other poles of $F(z)$, at points different from $z=m$. It follows that

$$
\sum_{m \in \mathbb{Z}} f(m)=-\left.\sum_{i} \operatorname{Res}[F(z)]\right|_{z=z_{i}} .
$$

In the particular case of the series,

$$
S(a)=\sum_{m \in \mathbb{Z}} \frac{1}{a^{2}+m^{2}},
$$

one considers the function $F(z)=\frac{\pi}{z^{2}+a^{2}} \cot (\pi z)$ which has two other simple poles in $z= \pm i a$ with residues

$$
\left.\operatorname{Res}\left[\frac{\pi}{z^{2}+a^{2}} \cot (\pi z)\right]\right|_{z= \pm i a}=-\frac{\pi}{2 a} \operatorname{coth}(\pi a) \text {. }
$$

Using (B.9), we thus have

$$
\sum_{m \in \mathbb{Z}} \frac{1}{a^{2}+m^{2}}=\frac{\pi}{a} \operatorname{coth}(\pi a)
$$

which implies that

$$
\begin{aligned}
\sum_{m \in \mathbb{Z}} \frac{1}{\left(a^{2}+m^{2}\right)^{s}} & =\frac{(-)^{s-1}}{2^{s-1}(s-1) !}\left(\frac{1}{a} \frac{\mathrm{d}}{\mathrm{d} a}\right)^{s-1} \sum_{m \in \mathbb{Z}} \frac{1}{a^{2}+m^{2}} \\
& =\frac{(-)^{s-1}}{2^{s-1}(s-1) !}\left(\frac{\mathrm{d}}{\mathrm{d} x}\right)^{s-1}\left(\operatorname{coth}(\pi \sqrt{2 x}) \frac{\pi}{\sqrt{2 x}}\right) \\
& =\sum_{m=0}^{s-1} \frac{\pi^{2 s-2 m-\frac{1}{2}}(-)^{m} \Gamma\left(s-\frac{1}{2}-m\right)}{2^{m} \Gamma(m+1) \Gamma(s-m)(\pi a)^{2 s-2 m-1}} \frac{d^{m}}{d x^{m}} \operatorname{coth}(\pi \sqrt{2 x}),
\end{aligned}
$$

where $x=\frac{1}{2} a^{2}$, and we used the Leibniz rule together with

$$
\frac{\mathrm{d}^{n}}{\mathrm{~d} x^{n}} x^{-\frac{1}{2}}=(-)^{n} \frac{\Gamma\left(\frac{1}{2}+n\right) x^{-\frac{1}{2}-n}}{\sqrt{\pi}},
$$

for $n=s-1-m$ have been used. In order to evaluate $\frac{\mathrm{d}^{n}}{\mathrm{~d} x^{n}} \operatorname{coth}(\pi \sqrt{2 x})$ we use formula (3n) of [55], which gives for $m \geq 1$,

$$
\frac{\mathrm{d}^{m}}{\mathrm{~d} x^{m}}(f \circ g)(x)=\sum_{r=1}^{m}\left(f^{(r)} \circ g\right)\left(\sum_{u=1}^{r} \frac{(-)^{r-u} g^{r-u}}{u !(r-u) !} \frac{\mathrm{d}^{m}}{\mathrm{~d} x^{m}} g^{u}\right),
$$


with $f(x)=\operatorname{coth} x$ and $g=\pi \sqrt{2 x}$ and where the exponent $(r)$ denotes the $r$-th derivative of the function with respect to its argument, and also

$$
\frac{\mathrm{d}^{m}}{d x^{m}} x^{\frac{u}{2}}=\frac{\Gamma\left(1+\frac{u}{2}\right) x^{\frac{u}{2}-m}}{\Gamma\left(1+\frac{u}{2}-m\right)} .
$$

When used together with the reduplication formula (2.22) at $z=u+1$, we get

$$
\begin{gathered}
\sum_{m \in \mathbb{Z}} \frac{1}{\left(a^{2}+m^{2}\right)^{s}}=\frac{\pi^{2 s}}{(\pi a)^{2 s-1}}\left[\frac{\Gamma\left(s-\frac{1}{2}\right)}{\pi^{\frac{1}{2}} \Gamma(s)} \operatorname{coth}(\pi a)+\sum_{m=1}^{s-1} \frac{(-)^{m} \Gamma\left(s-m-\frac{1}{2}\right)}{\Gamma(m+1) \Gamma(s-m)}\right. \\
\left.\sum_{r=1}^{m}(\pi a)^{r} \operatorname{coth}^{(r)}(\pi a)\left(\sum_{u=1}^{r} \frac{(-)^{r-u}}{2^{u} \Gamma\left(\frac{u+1}{2}\right) \Gamma(r-u+1) \Gamma\left(1+\frac{u}{2}-m\right)}\right)\right] .
\end{gathered}
$$

Note that the higher derivatives of $\operatorname{coth}(x)$ may be further expressed in terms of polynomials of $\operatorname{coth}(x)$. Even though we will not explicitly need it here, other useful relations are obtained by starting from (B.12) at $a=i l \tau$ to show that

$$
\sum_{m \in \mathbb{Z}} \frac{1}{(m+l \tau)}=\pi \cot (\pi l \tau)
$$

and then also

$$
\sum_{m \in \mathbb{Z}} \frac{1}{(m+l \tau)^{k}}=\frac{(-)^{k-1}}{(k-1) !} \frac{\pi}{l^{k-1}} \frac{\mathrm{d}^{k-1}}{\mathrm{~d} \tau^{k-1}} \cot (\pi l \tau) .
$$

The Epstein zeta function $\zeta\left(s ; \tau_{2}^{2}, 1\right)$ can be decomposed as

$$
\zeta\left(s ; \tau_{2}^{2}, 1\right)=\sum_{m \in \mathbb{Z}}^{\prime} \frac{1}{m^{2 s}}+\sum_{n \in \mathbb{Z}}^{\prime} \sum_{m \in \mathbb{Z}} \frac{1}{\left(n^{2} \tau_{2}^{2}+m^{2}\right)^{s}} .
$$

Applying the first of (B.13) to the last term in (B.20) with $a=n \tau_{2}$ gives,

$$
\zeta\left(s ; \tau_{2}^{2}, 1\right)=2 \zeta(2 s)+\frac{(-)^{s-1}}{2^{s-1}(s-1) !} \sum_{n \in \mathbb{Z}}^{\prime} \frac{1}{n^{2 s-1}}\left[\left(\frac{1}{\tau_{2}} \frac{\mathrm{d}}{\mathrm{d} \tau_{2}}\right)^{s-1} \frac{\pi}{\tau_{2}} \operatorname{coth}\left(\pi n \tau_{2}\right)\right] .
$$

Similarly, the analogous expansion for the real analytic Eisenstein series, which we give here without proof, is

$$
\tau_{2}^{-s} f_{s}(\tau, \bar{\tau})=2 \zeta(2 s)+\left[\frac{i(-)^{s-1}}{2^{s}(s-1) !} \sum_{n \in \mathbb{Z}}^{\prime} \frac{1}{n^{2 s-1}}\left(\frac{1}{\tau_{2}} \frac{\mathrm{d}}{\mathrm{d} \tau_{2}}\right)^{s-1} \frac{\pi}{\tau_{2}} \cot (\pi n \tau)+\text { c.c. }\right] .
$$

The right hand side of (B.22) correctly reduces to (B.21) when $\tau=i \tau_{2}$. Alternatively, one may use (B.17), to write

$$
\begin{gathered}
\zeta\left(s ; \tau_{2}^{2}, 1\right)=2 \zeta(2 s)+\sum_{n \in \mathbb{Z}}^{\prime} \frac{\pi^{2 s}}{\left(\pi n \tau_{2}\right)^{2 s-1}}\left[\frac{\Gamma\left(s-\frac{1}{2}\right)}{\pi^{\frac{1}{2}} \Gamma(s)} \operatorname{coth}\left(\pi n \tau_{2}\right)+\sum_{m=1}^{s-1} \frac{(-)^{m} \Gamma\left(s-m-\frac{1}{2}\right)}{\Gamma(m+1) \Gamma(s-m)}\right. \\
\left.\sum_{r=1}^{m}\left(\pi n \tau_{2}\right)^{r} \operatorname{coth}^{(r)}\left(\pi n \tau_{2}\right)\left(\sum_{u=1}^{r} \frac{(-)^{r-u}}{2^{u} \Gamma\left(\frac{u+1}{2}\right) \Gamma(r-u+1) \Gamma\left(1+\frac{u}{2}-m\right)}\right)\right], \quad \text { (B.23) }
\end{gathered}
$$


and also

$$
\begin{gathered}
\tau_{2}^{-s} f_{s}(\tau, \bar{\tau})=2 \zeta(2 s)+\sum_{n \in \mathbb{Z}}^{\prime} \frac{\pi^{2 s}}{2\left(\pi n \tau_{2}\right)^{2 s-1}}\left[\frac{\Gamma\left(s-\frac{1}{2}\right)}{\pi^{\frac{1}{2}} \Gamma(s)} i \cot (\pi n \tau)+\sum_{m=1}^{s-1} \frac{(-)^{m} \Gamma\left(s-m-\frac{1}{2}\right)}{\Gamma(m+1) \Gamma(s-m)}\right. \\
\left.\left.\sum_{r=1}^{m}\left(i \pi n \tau_{2}\right)^{r} i \cot ^{(r)}(\pi n \tau)\left(\sum_{u=1}^{r} \frac{(-)^{r-u}}{2^{u} \Gamma\left(\frac{u+1}{2}\right) \Gamma(r-u+1) \Gamma\left(1+\frac{u}{2}-m\right)}\right)+\text { c.c. }\right] . \quad \text { B. } 24\right)
\end{gathered}
$$

Let us briefly consider in more detail the simplest case of $d=3, s=2$, which is directly relevant for standard Casimir physics. In this case, (B.24) gives

$$
f_{2}(\tau, \bar{\tau})=\frac{\pi^{4} \tau_{2}^{2}}{2}\left[\frac{2}{45}+\sum_{l \in \mathbb{N}^{*}}\left(\frac{i \cot \pi l \tau}{\left(\pi l \tau_{2}\right)^{3}}-\frac{1}{\left(\pi l \tau_{2}\right)^{2} \sin ^{2} \pi l \tau}+\text { c.c. }\right)\right],
$$

while the partition function is

$$
\ln \mathcal{Z}_{3}(\tau, \bar{\tau})=\frac{\pi^{2} L_{1} L_{2} \tau_{2}}{4 L_{3}^{2}}\left[\frac{2}{45}+\sum_{l \in \mathbb{N}^{*}}\left(\frac{i \cot \pi l \tau}{\left(\pi l \tau_{2}\right)^{3}}-\frac{1}{\left(\pi l \tau_{2}\right)^{2} \sin ^{2} \pi l \tau}+\text { c.c. }\right)\right] .
$$

This could also have been obtained starting from the canonical approach. Indeed, the integral in (3.52) can be done by direct integrations by parts so that one finds instead of (3.53),

$$
\begin{aligned}
\ln \mathcal{Z}_{3}(\tau, \bar{\tau})= & \frac{\pi^{2} L_{1} L_{2} \tau_{2}}{90 L_{3}^{2}}+\frac{L_{1} L_{2}}{2 \pi\left(L_{3} \tau_{2}\right)^{2}} \zeta(3) \\
& +\frac{L_{1} L_{2}}{2 \pi \beta^{2}} \sum_{n_{3} \in \mathbb{N}^{*}} \sum_{l \in \mathbb{N}^{*}}\left[\frac{\left(1+2 \pi \ln _{3} \tau_{2}\right)}{l^{3}}\left(e^{2 \pi i n_{3} l \tau}+e^{-2 \pi i n_{3} l \bar{\tau}}\right)\right] .
\end{aligned}
$$

The above result now follows when using that $\zeta(3)=\sum_{l \in \mathbb{N}^{*}}\left(\frac{1}{2} l^{-3}+\frac{1}{2} l^{-3}\right)$, and that

$$
\begin{gathered}
\sum_{n_{3} \in \mathbb{N}^{*}} e^{2 \pi i n_{3} l \tau}+\frac{1}{2}=\frac{1}{1-e^{2 \pi i l \tau}}-\frac{1}{2}=\frac{i}{2} \cot \pi l \tau, \\
\sum_{n_{3} \in \mathbb{N}^{*}} 2 \pi n_{3} e^{2 \pi i n_{3} l \tau}=\frac{1}{i l} \frac{\mathrm{d}}{\mathrm{d} \tau} \frac{1}{1-e^{2 \pi i l \tau}}=-\frac{\pi}{2 \sin ^{2} \pi l \tau} .
\end{gathered}
$$

If the chemical potential $\alpha$ vanishes, $\tau=i \tau_{2}=i \frac{\beta}{L_{3}}$, we now have

$$
\zeta\left(2 ; \tau_{2}^{2}, 1\right)=\sum_{l, m}^{\prime} \frac{1}{\left(l^{2}+m^{2} \tau_{2}^{2}\right)^{2}}=\pi^{4}\left[\frac{1}{45}+\sum_{l \in \mathbb{N}^{*}}\left(\frac{\operatorname{coth} \pi l \tau_{2}}{\left(\pi l \tau_{2}\right)^{3}}+\frac{1}{\left(\pi l \tau_{2}\right)^{2} \sinh ^{2} \pi l \tau_{2}}\right)\right],
$$

and

$$
\ln \mathcal{Z}_{3}\left(\tau_{2}\right)=\frac{L_{1} L_{2} \tau_{2}}{2 \pi^{2} L_{3}^{2}} \zeta\left(2 ; \tau_{2}^{2}, 1\right)
$$

which is the form under which the result appears in the Casimir literature when subtraction is done only for the term containing the vacuum energy. 
Open Access. This article is distributed under the terms of the Creative Commons Attribution License (CC-BY 4.0), which permits any use, distribution and reproduction in any medium, provided the original author(s) and source are credited.

\section{References}

[1] N.A. Obers and B. Pioline, Eisenstein series and string thresholds, Commun. Math. Phys. 209 (2000) 275 [hep-th/9903113] [INSPIRE].

[2] P.M. Petropoulos and P. Vanhove, Gravity, strings, modular and quasimodular forms, arXiv: 1206.0571 [INSPIRE].

[3] F.L. Williams and K. Kirsten, eds., A Window into Zeta and Modular Physics, Cambridge University Press (2014).

[4] P. Fleig, H.P.A. Gustafsson, A. Kleinschmidt and D. Persson, Eisenstein Series and Automorphic Representations: With Applications in String Theory, Cambridge Studies in Advanced Mathematics, Cambridge University Press (2018), [https://doi.org/10.1017/9781316995860].

[5] J. Blümlein, C. Schneider and P. Paule eds., Elliptic Integrals, Elliptic Functions and Modular Forms in Quantum Field Theory, Texts \& Monographs in Symbolic Computation, Springer International Publishing (2019).

[6] J. Ambjørn and S. Wolfram, Properties of the Vacuum. 1. Mechanical and Thermodynamic, Annals Phys. 147 (1983) 1 [inSPIRE].

[7] G. Plunien, B. Müller and W. Greiner, The Casimir Effect, Phys. Rept. 134 (1986) 87 [INSPIRE].

[8] H. Gies, K. Langfeld and L. Moyaerts, Casimir effect on the worldline, JHEP 06 (2003) 018 [hep-th/0303264] [INSPIRE].

[9] G. Plunien, B. Müller and W. Greiner, Casimir Energy at Finite Temperature, Physica A $\mathbf{1 4 5}$ (1987) 202 [INSPIRE].

[10] E. Elizalde, S.D. Odintsov, A. Romeo, A.A. Bytsenko and S. Zerbini, Zeta regularization techniques with applications, World Scientific Publishing, Singapore (1994), [DOI] [INSPIRE].

[11] M. Bordag, G.L. Klimchitskaya, U. Mohideen and V.M. Mostepanenko, Advances in the Casimir effect, vol. 145 of Int. Ser. Monogr. Phys., Oxford University Press (2009).

[12] A. Cappelli and A. Coste, On the Stress Tensor of Conformal Field Theories in Higher Dimensions, Nucl. Phys. B 314 (1989) 707 [inSPIRE].

[13] A.A. Belavin, A.M. Polyakov and A.B. Zamolodchikov, Infinite Conformal Symmetry in Two-Dimensional Quantum Field Theory, Nucl. Phys. B 241 (1984) 333 [INSPIRE].

[14] J.L. Cardy, Operator Content of Two-Dimensional Conformally Invariant Theories, Nucl. Phys. B 270 (1986) 186 [INSPIRE].

[15] A. Cappelli, C. Itzykson and J.B. Zuber, Modular Invariant Partition Functions in Two-Dimensions, Nucl. Phys. B 280 (1987) 445 [INSPIRE].

[16] J.L. Cardy, Operator content and modular properties of higher dimensional conformal field theories, Nucl. Phys. B 366 (1991) 403 [INSPIRE]. 
[17] L. Dolan and C.R. Nappi, A modular invariant partition function for the five-brane, Nucl. Phys. B 530 (1998) 683 [hep-th/9806016] [INSPIRE].

[18] E. Shaghoulian, Modular forms and a generalized Cardy formula in higher dimensions, Phys. Rev. D 93 (2016) 126005 [arXiv:1508.02728] [INSPIRE].

[19] E. Shaghoulian, Modular Invariance of Conformal Field Theory on $S^{1} \times S^{3}$ and Circle Fibrations, Phys. Rev. Lett. 119 (2017) 131601 [arXiv:1612.05257] [InSPIRE].

[20] G.T. Horowitz and E. Shaghoulian, Detachable circles and temperature-inversion dualities for $\mathrm{CFT}_{d}$, JHEP 01 (2018) 135 [arXiv:1709.06084] [INSPIRE].

[21] G. Barnich, Black hole entropy from nonproper gauge degrees of freedom: The charged vacuum capacitor, Phys. Rev. D 99 (2019) 026007 [arXiv: 1806.00549] [INSPIRE].

[22] G. Barnich and M. Bonte, Soft degrees of freedom, Gibbons-Hawking contribution and entropy from Casimir effect, in Proceedings, 11th International Symposium on Quantum Theory and Symmetries (QTS2019): Montreal, Canada, July, 1-5, 2019, M.B. Paranjape, R. MacKenzie, Z. Thomova, P. Winternitz and W. Witczak-Krempa, eds., CRM Series in Mathematical Physics, 2021, [arXiv: 1912.12698] [INSPIRE].

[23] D. Deutsch and P. Candelas, Boundary Effects in Quantum Field Theory, Phys. Rev. D 20 (1979) 3063 [INSPIRE].

[24] F. Alessio and G. Barnich, Modular invariance in finite temperature Casimir effect, JHEP 10 (2020) 134 [arXiv: 2007.13334] [INSPIRE].

[25] F. Alessio, G. Barnich and M. Bonte, Gravitons in a Casimir box, JHEP 02 (2021) 216 [Erratum ibid. 03 (2021) 228] [arXiv:2011.14432] [INSPIRE].

[26] C. Lutken and F. Ravndal, A Symmetry in the Finite Temperature Casimir Effect, J. Phys. A 21 (1988) L793.

[27] F. Ravndal and D. Tollefsen, Temperature inversion symmetry in the Casimir effect, Phys. Rev. D 40 (1989) 4191 [INSPIRE].

[28] C. Wotzasek, On the Casimir Effect and the Temperature Inversion Symmetry, J. Phys. A 23 (1990) 1627 [INSPIRE].

[29] F. Ravndal and C. Wotzasek, Temperature inversion symmetry in the Gross-Neveu model, Phys. Lett. B 249 (1990) 266 [InSPIRE].

[30] L.S. Brown and G.J. Maclay, Vacuum stress between conducting plates: An Image solution, Phys. Rev. 184 (1969) 1272 [INSPIRE].

[31] A. Roberge and N. Weiss, Gauge Theories With Imaginary Chemical Potential and the Phases of QCD, Nucl. Phys. B 275 (1986) 734 [inSPIRE].

[32] M.G. Alford, A. Kapustin and F. Wilczek, Imaginary chemical potential and finite fermion density on the lattice, Phys. Rev. D 59 (1999) 054502 [hep-lat/9807039] [INSPIRE].

[33] F. Karbstein and M. Thies, How to get from imaginary to real chemical potential, Phys. Rev. D 75 (2007) 025003 [hep-th/0610243] [inSPIRE].

[34] S.W. Hawking, Zeta Function Regularization of Path Integrals in Curved Space-Time, Commun. Math. Phys. 55 (1977) 133 [InSPIRE].

[35] B.S. DeWitt, Quantum Field Theory in Curved Space-Time, Phys. Rept. 19 (1975) 295 [INSPIRE]. 
[36] J.S. Dowker and R. Critchley, Effective Lagrangian and Energy Momentum Tensor in de Sitter Space, Phys. Rev. D 13 (1976) 3224 [inSPIRE].

[37] J.I. Kapusta, Bose-Einstein Condensation, Spontaneous Symmetry Breaking, and Gauge Theories, Phys. Rev. D 24 (1981) 426 [inSPIRE].

[38] K. Kirsten, Spectral Functions in Mathematics and Physics, CRC Press (2001).

[39] Wikipedia contributors, Theta function, Wikipedia, the free encyclopedia, https://en.wikipedia.org/wiki/Theta_function\#Integral_representations, (2021).

[40] R. Balian and B. Duplantier, Electromagnetic Waves Near Perfect Conductors. 2. Casimir Effect, Annals Phys. 112 (1978) 165 [INSPIRE].

[41] L.H. Ford, Spectrum of the Casimir Effect, Phys. Rev. D 38 (1988) 528 [inSPIRE].

[42] J. Polchinski, Evaluation of the One Loop String Path Integral, Commun. Math. Phys. 104 (1986) 37 [INSPIRE].

[43] C. Itzykson and J.B. Zuber, Two-Dimensional Conformal Invariant Theories on a Torus, Nucl. Phys. B 275 (1986) 580 [InSPIRE].

[44] C. Itzykson and J. Drouffe, Statistical Field Theory. Volume 2: Strong coupling, Monte Carlo methods, conformal field theory, and random systems, Cambridge University Press (1989).

[45] P. Di Francesco, P. Mathieu and D. Senechal, Conformal field theory, Springer Verlag (1997).

[46] M. Henkel, Conformal Invariance and Critical Phenomena, Texts and monographs in physics, Springer (1999).

[47] C.G. Callan Jr., S.R. Coleman and R. Jackiw, A new improved energy-momentum tensor, Annals Phys. 59 (1970) 42 [inSPIRE].

[48] R. Arnowitt, S. Deser and C. Misner, The dynamics of general relativity, in Gravitation, an Introduction to Current Research, Wiley, New York, U.S.A. (1962), pp. 227-265.

[49] I. Gradshteyn and I. Ryzhik, 8-9 - special functions, in Table of Integrals, Series, and Products, I. Gradshteyn and I. Ryzhik, eds., Academic Press (1980), pp. 904-1080, DOI.

[50] A.C. Petkou and A. Stergiou, Dynamics of Finite-Temperature Conformal Field Theories from Operator Product Expansion Inversion Formulas, Phys. Rev. Lett. 121 (2018) 071602 [arXiv: 1806.02340] [INSPIRE].

[51] A.C. Petkou, Thermal one-point functions and single-valued polylogarithms, Phys. Lett. B $\mathbf{8 2 0}$ (2021) 136467 [arXiv:2105.03530] [INSPIRE].

[52] B.S. DeWitt, Quantum Theory of Gravity. 1. The Canonical Theory, Phys. Rev. 160 (1967) 1113 [INSPIRE].

[53] D. Giulini, What is the geometry of superspace?, Phys. Rev. D 51 (1995) 5630 [gr-qc/9311017] [INSPIRE].

[54] H.W. Hamber and R.M. Williams, On the measure in simplicial gravity, Phys. Rev. D 59 (1999) 064014 [hep-th/9708019] [INSPIRE].

[55] M. McKiernan, On the nth derivative of composite functions, Am. Math. Mon. 63 (1956) 331. 